\title{
THE MODULI SPACE OF CURVES, DOUBLE HURWITZ NUMBERS, AND FABER'S INTERSECTION NUMBER CONJECTURE
}

\author{
I. P. GOULDEN, D. M. JACKSON AND R. VAKIL
}

\begin{abstract}
We define the dimension $2 g-1$ Faber-Hurwitz Chow/homology classes on the moduli space of curves, parametrizing curves expressible as branched covers of $\mathbb{P}^{1}$ with given ramification over $\infty$ and sufficiently many fixed ramification points elsewhere. Degeneration of the target and judicious localization expresses such classes in terms of localization trees weighted by "top intersections" of tautological classes and genus 0 double Hurwitz numbers. This identity of generating series can be inverted, yielding a "combinatorialization" of top intersections of $\psi$-classes. As genus 0 double Hurwitz numbers with at most 3 parts over $\infty$ are well understood, we obtain Faber's Intersection Number Conjecture for up to 3 parts, and an approach to the Conjecture in general (bypassing the Virasoro Conjecture). We also recover other geometric results in a unified manner, including Looijenga's theorem, the socle theorem for curves with rational tails, and the hyperelliptic locus in terms of $\kappa_{g-2}$.
\end{abstract}

\section{Part 1. INTRODUCTION AND SUMMARY OF RESULTS}

Since we shall be using arguments from geometry and combinatorics, we have separated the material into three parts to assist the reader. Part 1 gives the background to the topic and a summary of our results. Part 2 contains the geometry that uses degeneration to obtain a recursion for the Faber-Hurwitz classes, and localization to express these as tree sums involving the Faber symbol. Part 3 contains an approach through algebraic combinatorics to transform and then solve the formal partial differential equations and functional equations that originate from degeneration and localization in Part 2 and thence to obtain the top intersection numbers. We have sought to make the transition from the geometry of Part 2 to the combinatorics of Part 3 pellucid.

\section{Summary of Results}

The purpose of this paper is to give a geometrico-combinatorial approach that is direct and, we hope, enlightening, to the three known results that are listed below. We give a summary of results for those quite familiar with moduli spaces of curves, and Faber's foundational conjectures on their cohomology or Chow rings. A more detailed introduction to the paper is given in Section 2, and most readers should turn immediately to this.

The three results are:

(I) $R_{2 g-1}\left(\mathcal{M}_{g, n}^{r t}\right)$ is generated by a single element, which we denote $\mathbb{G}_{g, 1}$ (Theorem 3.12). This argument was promised in [GV3, Sec. 5.7]. (Since $R_{2 g-1}\left(\mathcal{M}_{g}\right)$ was shown earlier by Faber to be non-zero [F1, Thm. 2], this single element is also non-zero by Remark 2.3(iii) below.)

(II) A combinatorial description of $\psi_{1}^{a_{1}} \cdots \psi_{n}^{a_{n}} \in R_{2 g-1}\left(\mathcal{M}_{g, n}^{r t}\right)$ as a multiple of this generator, in terms of genus 0 double Hurwitz numbers (Theorem 3.11). (These intersections of $\psi$ classes determine all top intersections in the tautological ring, and are the subject of Faber's Intersection Number Conjecture.)

Date: Tuesday, November 21, 2006.

The first two authors are partially supported by NSERC grants. The third author is partially supported by NSF PECASE/CAREER grant DMS-0238532.

2000 Mathematics Subject Classification: Primary 14H10, Secondary 05E99, 14K30. 
(III) Hence a proof of Faber's Intersection Number Conjecture for up to three points, and arbitrary genus (Theorem 2.5).

Past proofs of some of these results are described in Section 1.2. In the above statements we have used the following notation. Let $\mathcal{M}_{g, n}^{r t}$ be the moduli space of $n$-pointed genus $g$ stable curves with "rational tails", and $R^{*}\left(\mathcal{M}_{g, n}^{r t}\right)$ its tautological ring. Genus 0 double Hurwitz numbers enumerate branched covers of the sphere by another sphere, with branching over 0 and $\infty$ specified by partitions $\alpha$ and $\beta$ respectively, and the simplest non-trivial branching over an appropriate number of other given points. They are well understood in the case where one of the partitions has at most three parts GJV2].

The "one-part analysis" yields as corollaries new proofs of a number of important facts, such as the class of the hyperelliptic locus in $\mathcal{M}_{g}$ as a multiple of $\kappa_{g-2}$. These results are collected as Corollaries 6.3 through 6.6. A direct proof of these consequences by our methods would be much shorter; much of our effort will be to develop techniques to deal with more parts.

We note that Faber's Intersection Number Conjecture for bounded genus involves a finite amount of information, thanks to the string and dilaton equation (Prop. 2.6); for any (reasonably small) genus, this finite amount of information can be directly computed [F3]. (We point out that although Faber's verification of his conjecture up to genus 21, [F4], using work of Pandharipande, involves a finite amount of information, it is a large amount, and is very difficult.) However, Faber's Intersection Number Conjecture for a bounded number of points, the case considered here, involves an infinite amount of information.

The methods here can readily be adapted to deal with a larger number of points. For example, the case $n=4$ follows from the formulae for genus 0 double Hurwitz numbers from [GJV2, and a Maple computation. The case $n=5$ should also be computationally tractable. However, we content ourselves with calculations that could be done by hand, as the route to a natural proof of Faber's Intersection Number Conjecture is clearly not through any hoped-for closed form description of the genus 0 double Hurwitz generating series in general — such series might be expected to get quite complicated. Instead, we hope for a proof using the structure of the double Hurwitz generating series as a whole, and there are indications that this may be tractable. We point out in particular the very recent preprint [SSV], giving a good description of genus 0 double Hurwitz numbers in general, and a particularly elegant description in many cases.

1.1. Motivation. The ELSV formula provides a remarkable link between Hurwitz numbers (counting branched covers of $\mathbb{P}^{1}$ or, combinatorially, transitive factorizations of elements of the symmetric group into transpositions) and the intersection theory on the moduli space of curves. See [ELSV1, ELSV2], and [GV2 for a proof in the context of Gromov-Witten theory. The ELSV formula describes Hurwitz numbers in terms of top intersections of the moduli space of curves. This relation can be "inverted" to prove results on top intersections on the moduli space of curves. This has been used by a large number of authors, including Ekedahl, Kazarian, Lando, Okounkov, Pandharipande, Shadrin, Shapiro, Vainshtein and Zvonkine, to great effect. Notable examples are the proofs of Witten's Conjecture $\mathrm{OP}, \mathrm{KL}$.

This paper relies on the observation that similar methods can be applied to the (non-compact) moduli space of smooth curves. In this case, localization turns question about top intersections into "genus 0 combinatorics" (involving trees rather than general graphs), which should in principle be simpler than the genus $g$ combinatorics that arises in the case of compactified moduli space. Indeed, we immediately get some results (for example, results (I) and (II)) from the statement of the analogue of the ELSV formula. Explicitly inverting this ELSV-analogue requires more work, but then our knowledge of genus 0 double Hurwitz numbers with few parts translates directly into Faber's Intersection Number Conjecture with few parts (result (III)).

We point out that these techniques of algebraic combinatorics are useful in geometry in a wider context. A further example of their use appears in GJV3] where we give a direct proof of Getzler 
and Pandharipande's $\lambda_{g}$-Conjecture (without Gromov-Witten theory), and related techniques have been used by a number of the authors mentioned above.

1.2. Background. The one-dimensionality of (I) was established in [FP], and may also follow from Looijenga $[\mathrm{Lo}]$. The argument given here can be seen as an extension of the tautological vanishing theorem of [GV3. The entire argument is outlined in a few pages in [V2, Sec. 7].

Getzler and Pandharipande showed that Faber's Intersection Number Conjecture is a formal consequence of the Virasoro Conjecture for the projective plane, $\mathrm{GeP}$, in fact just the degree 0 part (the "large volume" limit). Givental proved the Virasoro Conjecture for projective space (and more generally Fano toric manifolds) Gi1, Gi2]. Y.-P. Lee and Pandharipande are writing a book LP giving details. Givental's result is one of the most important results in Gromov-Witten theory, and is a marvelous feat. However, it seems circuitous to prove the Intersection Number Conjecture by means of the Virasoro Conjecture. The latter is a very heavy instrument which conceals the combinatorial structure that lies behind the intersection numbers. As noted by K. Liu and Xu $[\mathrm{LX}$, it is very desirable to have a shorter and direct explanation. (Liu and $\mathrm{Xu}$ show how the conjecture cleanly follows from another attractive conjectural identity.) For this reason, we give such an argument, paralleling our understanding of top intersection numbers on $\overline{\mathcal{M}}_{g, n}$ via Hurwitz numbers.

1.3. Outline of paper. The strategy of the paper is as follows. We define dimension $2 g-1$ Faber-Hurwitz Chow/homology classes on the moduli space of curves, by considering ("virtually") branched covers of $\mathbb{P}^{1}$ with given ramification over $\infty$ and sufficiently many fixed ramification points elsewhere. We consider only curves with rational tails, which simplifies the combinatorics dramatically. We use the two most effective techniques of Gromov-Witten theory, degeneration and localization.

- Degeneration of the target yields a recursion for such classes, which we can solve explicitly. The story is analogous to that of genus 0 Hurwitz numbers (which are combinatorially straightforward), not genus $g$ Hurwitz numbers.

- Localization expresses such classes in terms of the desired top intersections and genus 0 double Hurwitz numbers. The rational tails constraint forces the resulting localization graphs to be trees.

More precisely, localization expresses Faber-Hurwitz classes as a sum over certain decorated trees (Sec. 3.10) of linear combinations of top intersections (including those that are the subject of Faber's conjecture) and genus 0 double Hurwitz numbers. The relation between the top intersections and Faber-Hurwitz classes can be easily seen to be invertible, i.e. the top intersections are linear combinations of Faber-Hurwitz classes (which we already understood via degeneration). From this, Looijenga's Theorem drops out quickly (from Theorem 3.12), for example. The central idea of this paper is that this inversion may be done explicitly: the localization sum gives an expression which readily can be unwound, and from which the top intersections can be extracted. This is done by a change of variables.

Using this approach, we quickly recover various geometric results in a unified manner (Sec. 6.2). Also, as genus 0 double Hurwitz numbers with at most 3 parts over $\infty$ are well understood, we obtain Faber's Intersection Number Conjecture for up to 3 parts, and an approach to the conjecture in general.

In Section 2 we state the Faber Intersection Number Conjecture and give the geometric and algebraic combinatorics background to our approach. The Faber-Hurwitz classes are defined in Section 3, and we obtain a Join-cut Recursion for them by degeneration of the target. This is the first form of the Degeneration Theorem, and the equivalent Join-cut (partial differential) Equation for the Faber-Hurwitz series is given as the second form of this Theorem. In addition, using localization, we obtain an expression for the Faber-Hurwitz classes as a weighted sum over (localization) trees, giving the first form of the Localization Tree Theorem. Section 4 addresses the weighted tree sum, using the combinatorics of rooted, labelled trees and exponential generating series in a countable 
set of indeterminates. This results in the second form of the Localization Tree Theorem, which is purely algebraic, giving an expression for the Faber-Hurwitz series in terms of Faber's intersection numbers and the unique solution to a functional equation. Section 5 introduces the fundamental transformation, as the composition of three operators. The first two operators are a symmetrization and an implicit change of variables, which gives polynomials in the new indeterminates. The third operator restricts these polynomials to "top" terms - those of maximum total degree. Our strategy of proof for Faber's Intersection Number Conjecture is to apply the fundamental transformation to the Localization Tree Theorem and to the Degeneration Theorem, and eliminate the transformed Faber-Hurwitz series. The key to the proof is that only top intersection numbers remain when we do so, and they appear in enough linearly independent equations to uniquely determine them. Section 6 applies the strategy for the first time, to the one-part case (of Faber top intersection numbers) which has some non-trivial geometric consequences. The methodology to that point requires us to consider an equation for each genus $g$ separately, so in Section 7 we refine the methodology by creating generating series in genus. This means that a single generating series equation suffices to prove Faber's Intersection Number Conjecture for each number of parts. In Section 8, we apply this refined methodology to establish Faber's (top) Intersection Number Conjecture for 2 and 3 parts, and we include remarks about the general case.

Appendix $B$ is a glossary of notation for the reader's convenience.

Acknowledgments. The third author has benefited from discussions with Renzo Cavalieri, Carel Faber, Y.-P. Lee, Rahul Pandharipande, Hsian-Hua Tseng, and especially Tom Graber, who developed many of the algebro-geometric foundations on which this paper is based. We also thank Ezra Getzler, Kefeng Liu, and Melissa Liu.

\section{INTRODUCTION}

2.1. Geometric background. We work over the complex numbers. Throughout, the genus $g$ is at least 1. We assume some knowledge of the moduli space of curves. An overview is given in [V2], which outlines the necessary background and ends with a sketch of many of the results of this paper; see also [V1]. We also assume familiarity with Gromov-Witten theory, in particular the theory of relative stable maps, and with localization on their moduli space ("relative virtual localization") [Li1, Li2, GV3, LLZ]. An introduction to many of the Gromov-Witten ideas we shall use may be found in $\left[\mathrm{V} 2\right.$ and $[\mathrm{H}]$. We prefer to work in the Chow ring $A^{*}$ rather than the cohomology ring $H^{2 *}$, because our arguments apply in this more refined setting, but there is no loss should the reader wish to work in cohomology. Chow/homology classes will often be written in blackboard bold font $(e . g . \mathbb{F})$ in order to distinguish them from numbers.

Faber's conjectures on the topology of the moduli space of smooth curves $\mathcal{M}_{g}$ (given in [F1]) are a striking description of the "tautological" part of the cohomology ring, the part of the cohomology ring arising "naturally from geometry". The "top intersections" in this ring have a particularly remarkable combinatorial structure. We begin by describing these conjectures.

On the moduli space of stable $n$-pointed genus $g$ stable curves $\overline{\mathcal{M}}_{g, n}$ (or any open subset thereof), let $\psi_{i}(1 \leq i \leq n)$ be the first Chern class of the line bundle corresponding to the cotangent space of the universal curve at the $i$ th marked point.

We shall denote all forgetful morphisms $\overline{\mathcal{M}}_{g, n} \rightarrow \overline{\mathcal{M}}_{g, n^{\prime}}$ by $\pi$, where the source and target will be clear from the context. For example if $\pi: \mathcal{M}_{g, 1} \rightarrow \mathcal{M}_{g}$, then the $i$ th Mumford-Morita-Miller " $\kappa$-class" is defined by $\kappa_{i}:=\pi_{*} \psi_{1}^{i+1}$.

Given an $\left(n_{1}+1\right)$-pointed curve of genus $g_{1}$, and an $\left(n_{2}+1\right)$-pointed curve of genus $g_{2}$, gluing the first curve to the second along the last point of each yields an $\left(n_{1}+n_{2}\right)$-pointed curve of genus $g_{1}+g_{2}$. This gives a map

$$
\overline{\mathcal{M}}_{g_{1}, n_{1}+1} \times \overline{\mathcal{M}}_{g_{2}, n_{2}+1} \rightarrow \overline{\mathcal{M}}_{g_{1}+g_{2}, n_{1}+n_{2}} .
$$


Similarly, we can take a single $(n+2)$-pointed curve of genus $g$, and glue its last two points together to get an $n$-pointed curve of genus $g+1$. This gives a map

$$
\overline{\mathcal{M}}_{g, n+2} \rightarrow \overline{\mathcal{M}}_{g+1, n}
$$

We call these two types of maps gluing morphisms. We call the forgetful and gluing morphisms the natural morphisms between moduli spaces of curves.

2.2. The tautological ring. There are many equivalent definitions of the tautological ring in the literature. The following will be convenient for our purposes.

Definition 2.1. GV3, Def. 4.2] The system of tautological rings $\left(R^{*}\left(\overline{\mathcal{M}}_{g, n}\right) \subset A^{*}\left(\overline{\mathcal{M}}_{g, n}\right)\right)_{g, n}$ is the smallest system of $\mathbb{Q}$-vector spaces closed under pushforwards by the natural morphisms, such that all monomials in $\psi_{1}, \ldots, \psi_{n}$ lie in $R^{*}\left(\overline{\mathcal{M}}_{g, n}\right)$.

If $\mathcal{M}$ is an open subset of $\overline{\mathcal{M}}_{g, n}$, let $R^{*}(\mathcal{M}):=\left.R^{*}\left(\mathcal{M}_{g, n}\right)\right|_{\mathcal{M}}, R_{j}(\mathcal{M}):=R^{\operatorname{dim} \mathcal{M}-j}(\mathcal{M})$. (Here $R^{k}(\mathcal{M})$ is the codimension $k$ part of $R^{*}(\mathcal{M})$.) We shall often use the notation $R_{*}$ instead of $R^{*}$, because we wish to think of classes as homology classes.

We take this opportunity to introduce a third sort of tautological class: let $\mathbb{E}_{g, n}$ be the Hodge bundle on $\overline{\mathcal{M}}_{g, n}$. It has rank $g$, and

$$
\pi^{*} \mathbb{E}_{g, 0}=\mathbb{E}_{g, n}
$$

where $\pi$ is the forgetful morphism $\pi: \overline{\mathcal{M}}_{g, n} \rightarrow \overline{\mathcal{M}}_{g}$. Over a point $\left[\left(C, p_{1}, \ldots, p_{n}\right)\right] \in \mathcal{M}_{g, n}$, the fiber of $\mathbb{E}_{g, n}$ is the vector space of differentials on $C$. The $\lambda$-classes are defined by $\lambda_{i}=c_{i}\left(\mathbb{E}_{g, n}\right)$. By the above relation, they behave well with respect to pullback by forgetful morphisms ( $\pi^{*} \lambda_{i}=\lambda_{i}$ "). Note that $\lambda_{0}=1$.

It is not hard to show that the tautological ring of the moduli space of smooth curves $\mathcal{M}_{g}$ is generated by the $\kappa$-classes, and indeed this is essentially the original definition of $R^{*}\left(\mathcal{M}_{g}\right)$ in [F1].

We now describe three predictions of Faber, namely the Vanishing Conjecture, the Perfect Pairing Conjecture, and the Intersection Number Conjecture, which is a central subject of this paper.

Vanishing Conjecture. $R^{i}\left(\mathcal{M}_{g}\right)=0$ for $i>g-2$, and $R^{g-2}\left(\mathcal{M}_{g}\right) \cong \mathbb{Q}$. This was proved by Looijenga and Faber. Looijenga's Theorem [Lo] is that $R^{g-2}\left(\mathcal{M}_{g}\right)$ is generated as a vector space by a single element (this will also follow from our analysis, see Thm. 3.12). Faber proved the following "non-vanishing theorem".

Theorem 2.2 (Faber [F1], Thm. 2). $R^{g-2}\left(\mathcal{M}_{g}\right) \neq 0$.

(For other proofs, see [BP, Thm. 6.5], and [BCT, Thm. 0.2], which is based on [C]. More precisely, Faber described a linear functional $\int \cdot \lambda_{g-1} \lambda_{g}: R^{g-2}\left(\mathcal{M}_{g}\right) \rightarrow \mathbb{Q}$, and showed that the image was nonzero by computing a certain intersection number. Bryan-Pandharipande [BP] and later BertramCavalieri-Todorov [BCT] show the image is non-zero by different enlightening computations.)

Perfect Pairing Conjecture. The analogue of Poincaré duality holds: for $0 \leq i \leq g-2$, the cup product $R^{i}\left(\mathcal{M}_{g}\right) \times R^{g-2-i}\left(\mathcal{M}_{g}\right) \rightarrow R^{g-2}\left(\mathcal{M}_{g}\right) \cong \mathbb{Q}$ is a perfect pairing. This conjecture is known only in special cases, and is essentially completely open.

Informally, these two conjectures state that " $R^{*}\left(\mathcal{M}_{g}\right)$ behaves like the $((p, p)$-part of the) cohomology ring of a $(g-2)$-dimensional complex projective manifold." They imply that the entire structure of the ring is determined by the top intersections of the $\kappa$-classes, i.e. by $\prod \kappa_{d_{i}}$ (where $\left.\sum_{i} d_{i}=g-2\right)$ in terms of some fixed generator of $R_{2 g-1}\left(\mathcal{M}_{g}\right)$.

Faber's Intersection Number Conjecture. This gives a combinatorial description of these top intersections. We note that this conjecture is useful even without knowing the perfect pairing conjecture; Faber's algorithm [F2] reduces all "top intersections" in the tautological ring to intersections of the form described in his Intersection Number Conjecture.

Faber reformulated his conjecture in the striking form given in Conjecture 2.4. In order to give this reformulation, we review the extension of Faber's Conjecture to curves with "rational tails" 
(by Faber and Pandharipande, see for example [P2]). Recall that a nodal genus $g$ nodal curve is said to be a genus $g$ curve with rational tails if one component is a smooth curve of genus $g$, and hence the remaining components are genus 0 (spheres), see Figure1. Then the dual graph is a tree, a fact which will prove crucial for us. This is the reason that the graphs arising from localization involves just tree combinatorics.

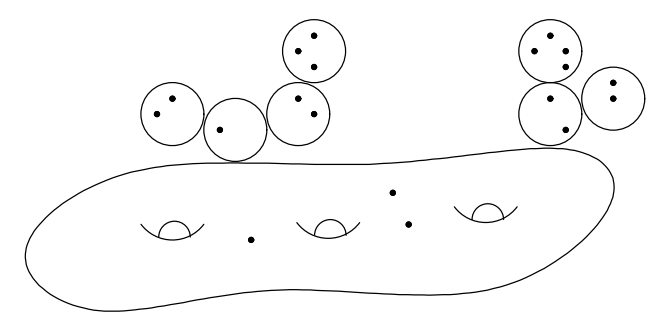

FIgURE 1. A pointed curve with "rational tails". Notice that the dual graph is a tree.

Denote the corresponding open subset of $\overline{\mathcal{M}}_{g, n}$ (corresponding to stable curves with rational tails) by $\mathcal{M}_{g, n}^{r t}$. If $\pi: \overline{\mathcal{M}}_{g, n} \rightarrow \overline{\mathcal{M}}_{g}$, then $\mathcal{M}_{g, n}^{r t}=\pi^{-1}\left(\mathcal{M}_{g}\right)$. In particular, the forgetful morphism $\mathcal{M}_{g, n}^{r t} \rightarrow \mathcal{M}_{g, n^{\prime}}^{r t}$ for $n \geq n^{\prime}$ is projective, and $\mathcal{M}_{g, 1}^{r t}=\mathcal{M}_{g, 1}$.

We recall some background.

Remark 2.3. $\quad$ i) $R_{j}\left(\mathcal{M}_{g, n}^{r t}\right)=0$ for $j<2 g-1$ and all $n$;

ii) for $n \geq 1$,

$$
\pi_{*}: R_{2 g-1}\left(\mathcal{M}_{g, n}^{r t}\right) \rightarrow R_{2 g-1}\left(\mathcal{M}_{g, 1}^{r t}\right)=R_{2 g-1}\left(\mathcal{M}_{g, 1}\right)
$$

(where $\pi: \mathcal{M}_{g, n}^{r t} \rightarrow \mathcal{M}_{g, 1}$ is the forgetful morphism) is an isomorphism; and

iii) $\pi_{*}: R_{2 g-1}\left(\mathcal{M}_{g, 1}\right) \rightarrow R_{2 g-1}\left(\mathcal{M}_{g}\right)$ (where $\pi: \mathcal{M}_{g, 1} \rightarrow \mathcal{M}_{g}$ is the forgetful morphism) is surjective.

Statements (i) and (ii) are parts (a) and (b) of [GV3, Prop. 5.8]. Statement (iii) is immediate from an appropriate definition of the tautological ring, for example Definition 2.1.

Conjecture 2.4 (Faber's Intersection Number Conjecture, " $\psi$-form"). For any $n$-tuple of positive integers $\left(d_{1}, \ldots, d_{n}\right)$,

$$
\pi_{*}\left(\psi_{1}^{d_{1}} \cdots \psi_{n}^{d_{n}}\right)=\frac{(2 g-3+n) !(2 g-1) ! !}{(2 g-1) ! \prod_{j=1}^{n}\left(2 d_{j}-1\right) ! !} \psi_{1}^{g-1} \quad \text { for } \sum_{i} d_{i}=g-2+n
$$

where $\pi$ is the forgetful morphism $\mathcal{M}_{g, n}^{r t} \rightarrow \mathcal{M}_{g, 1}$. (Recall that $(2 a-1) ! !=1 \cdot 3 \cdots(2 a-1)=$ $\left.(2 a) ! /\left(2^{a} a !\right).\right)$

Note that Faber's Intersection Number Conjecture is immediate for the case $n=1$. We shall prove the following.

Theorem 2.5. Faber's Intersection Number Conjecture 2.4 is true for up to 3 points (i.e. for $n \leq 3)$.

We shall need to consider more general intersection numbers involving one $\lambda$-class, arising in the virtual localization formula. Motivated by the isomorphism (1), and in analogy with the "Witten symbol" used in Gromov-Witten theory (e.g. [H, Sec. 26.2]), define the Faber symbol as

$$
\left\langle\tau_{a_{1}} \cdots \tau_{a_{n}} \lambda_{k}\right\rangle_{g}^{\mathbb{F} \text { ab }}:=\pi_{*}\left(\psi_{1}^{a_{1}} \cdots \psi_{n}^{a_{n}} \lambda_{k}\right) \in R_{2 g-1}\left(\mathcal{M}_{g, 1}\right)
$$

where the pushforward $\pi_{*}$ is the isomorphism of (1) $\left(\pi: \mathcal{M}_{g, n}^{r t} \rightarrow \mathcal{M}_{g, 1}\right.$ is the forgetful morphism). Caveat: this symbol is a Chow class, not a number; we repeat that we will often indicate classes 
using blackboard bold font. The symbol is declared to be zero if some $a_{i}<0$, or if the product lies in wrong degree, i.e.

$$
k+\sum_{i} a_{i} \neq \operatorname{dim} \mathcal{M}_{g, n}^{r t}-(2 g-1)=g-2+n .
$$

Note that the case $k=0$ is equivalent to there being no $\lambda$-factor, as $\lambda_{0}=1$. This symbol satisfies a version of the usual string and dilaton equations:

Proposition 2.6. The following relations among the Faber symbols hold.

$$
\begin{aligned}
& \left\langle\tau_{0} \tau_{a_{1}} \cdots \tau_{a_{n}} \lambda_{k}\right\rangle_{g}^{\mathbb{F a b}}=\sum_{i=1}^{n}\left\langle\tau_{a_{1}} \cdots \tau_{a_{i}-1} \cdots \tau_{a_{n}} \lambda_{k}\right\rangle_{g}^{\mathbb{F a b}} \quad \text { (string equation), } \\
& \left\langle\tau_{1} \tau_{a_{1}} \cdots \tau_{a_{n}} \lambda_{k}\right\rangle_{g}^{\mathbb{F a b}}=(2 g-2+n)\left\langle\tau_{a_{1}} \cdots \tau_{a_{n}} \lambda_{k}\right\rangle_{g}^{\mathbb{F a b}} \quad \text { (dilaton equation). }
\end{aligned}
$$

The proof is a variation of the proof of the usual string and dilaton equations, and is left as an exercise to the reader (see for example [V2, Sec. 3.13]).

\section{Part 2. GEOMETRY}

In Part 2, we use degeneration and localization to obtain a topological recursion for the FaberHurwitz classes, and an expression for the Faber-Hurwitz classes as a sum over a class of weighted trees that are to be defined. The topological recursion is then transformed into a partial differential equation for the Faber-Hurwitz series.

\section{Degeneration and localization}

For a partition $\alpha$, we use $|\alpha|$ and $l(\alpha)$ for the sum of parts and number of parts, respectively, and write $\alpha \vdash|\alpha|$. If $\alpha$ has $i_{j}$ parts equal to $j, j \geq 1$, then we also write $\alpha=\left(1^{i_{1}} 2^{i_{2}} \cdots\right)$, where convenient. The set of all non-empty partitions is denoted by $\mathcal{P}$.

3.1. Relative stable maps. We shall use the theory of stable relative maps to $\mathbb{P}^{1}$, following J. Li's algebro-geometric description in [Li1], and his description of their deformation-obstruction theory in [Li2]. (We point out earlier definitions of relative stable maps in the differentiable category due to A.-M. Li and Y. Ruan [LR], and Ionel and Parker [IP1, IP2], and Gathmann's work Ga] in the algebraic category in genus 0 .) We need the algebraic category for several reasons, most importantly because we shall use virtual localization, and an explicit description of the moduli space's deformation-obstruction theory.

3.1.1. Relative to one point. The moduli space of genus $g$ relative stable maps to $\mathbb{P}^{1}$ relative to one point $\infty$, with branching above $\infty$ given by the partition $\alpha \vdash d$, is denoted by $\overline{\mathcal{M}}_{g, \alpha}\left(\mathbb{P}^{1}\right)$ where $d$ is the degree of the cover.

A relative map to $X=\mathbb{P}^{1}$ is the following data:

- a morphism $f_{1}$ from a nodal $n$-pointed genus $g$ curve $\left(C, q_{1}, \ldots, q_{n}\right)$ (where as usual the $q_{j}$ are distinct non-singular points) to a chain of $\mathbb{P}^{1}$ 's, $T=T_{0} \cup T_{1} \cup \cdots \cup T_{t}$ (where $T_{i}$ and $T_{i+1}$ meet), with a point $\infty \in T_{t}-T_{t-1}$, so there are two points named $\infty$. We will call the one on $X, \infty_{X}$, and the one on $T, \infty_{T}$, whenever there is any ambiguity.

- A projection $f_{2}: T \rightarrow X$ contracting $T_{i}$ to $\infty_{X}$ (for $i>0$ ) and giving an isomorphism from $\left(T_{0}, T_{0} \cap T_{1}\right)$ (resp. $\left.\left(T_{0}, \infty_{T}\right)\right)$ to $\left(X, \infty_{X}\right)$ if $t>0$ (resp. if $\left.t=0\right)$. Denote $f_{2} \circ f_{1}$ by $f$.

- We have an equality of divisors on $C: f_{1}^{*} \infty_{T}=\sum \alpha_{i} q_{i}$. In particular, $f_{1}^{-1} \infty_{T}$ consists of non-singular marked points of $C$. 
- The preimage of each node $n$ of $T$ is a union of nodes of $C$. At any such node $n^{\prime}$ of $C$, the two branches map to the two branches of $n$, and their orders of branching are the same. This is called the predeformability or kissing condition. See Figure 2 for a pictorial representation. Analytically, this map is of the following form. The node $u v=0$ in the $u v$-plane maps to the node $x y=0$ in the $x y$-plane by $(u, v) \mapsto\left(u^{m}, v^{m}\right)=(x, y)$. (The branching of the $u$-axis over the $x$-axis is the same as the branching of the $v$-axis over the $y$-axis.)

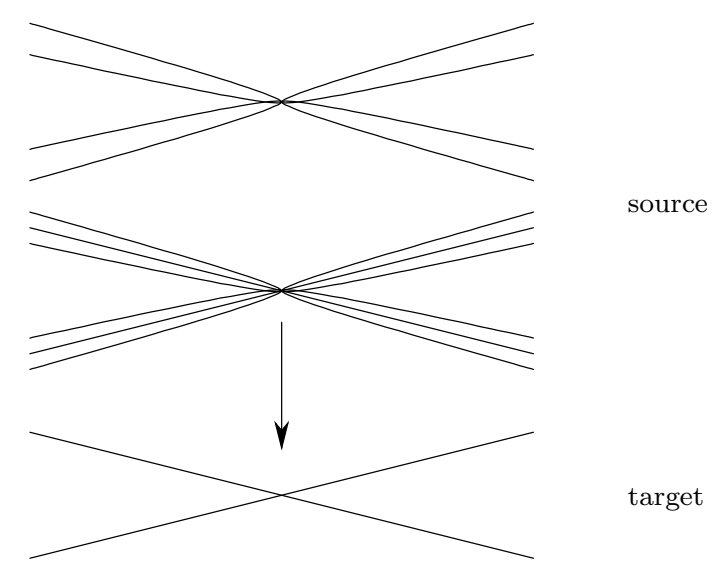

Figure 2. The "predeformability" or "kissing" condition on maps of nodes to nodes. The singularities of the source and target are nodes (analytically isomorphic to $x y=0$ in $\mathbb{C}^{2}$ ), although it is impossible to depict them as such on the page.

An isomorphism of two such maps is a commuting diagram

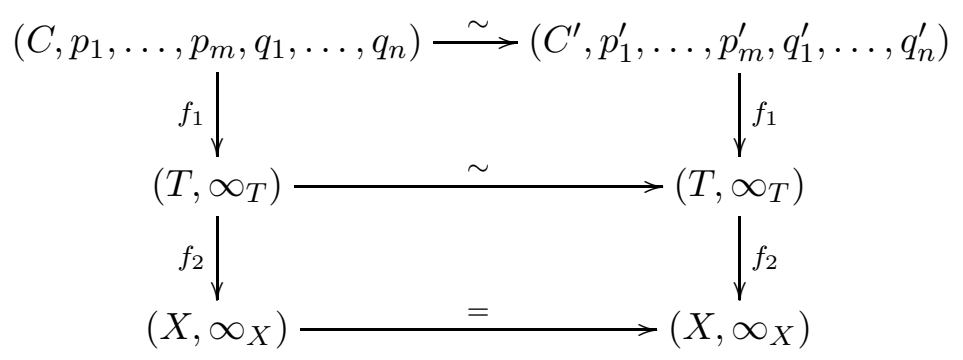

where all horizontal morphisms are isomorphisms, the bottom (although not necessarily the middle!) is an equality, the top horizontal isomorphism sends $p_{i}$ to $p_{i}^{\prime}$ and $q_{j}$ to $q_{j}^{\prime}$. Note that the middle isomorphism must preserve the isomorphism of $T_{0}$ with $X$, and is hence the identity on $T_{0}$, but for $i>0$, the isomorphism may not be the identity on $T_{i}$.

We say that $f$ is stable if it has finite automorphism group.

Let $r_{\alpha}^{g}$ be the "expected" number of branch points away from $\infty$ (the number if all the branching over points other than $\infty$ - "away from $\infty$ " - were simple, i.e. corresponding to the partition $\left.\left(1^{d-2} 2\right) \vdash d\right)$. Then

$$
r_{\alpha}^{g}:=d+l(\alpha)+2 g-2,
$$

by the Riemann-Hurwitz formula. Then $\overline{\mathcal{M}}_{g, \alpha}\left(\mathbb{P}^{1}\right)$ supports a virtual fundamental class

$$
\left[\overline{\mathcal{M}}_{g, \alpha}\left(\mathbb{P}^{1}\right)\right]^{\mathrm{vir}} \in A_{r_{\alpha}^{g}}\left(\overline{\mathcal{M}}_{g, \alpha}\left(\mathbb{P}^{1}\right)\right)
$$

of dimension $r_{\alpha}^{g}$. 
There is a Fantechi-Pandharipande branch morphism [FnP]

$$
\text { br }: \overline{\mathcal{M}}_{g, \alpha}\left(\mathbb{P}^{1}\right) \rightarrow \operatorname{Sym}^{r_{\alpha}^{g}}\left(\mathbb{P}^{1}\right)
$$

sending each map to the set of its branch points in $\mathbb{P}^{1}$, excluding the ones that are "automatic" because of the branching over $\infty$. Note that the total branching $C \rightarrow \mathbb{P}^{1}$ above a point in the target $p \neq \infty \in \mathbb{P}^{1}$ is 1 only if the map is simply branched above $p$.

3.1.2. Relative to two points. Similarly, let $\overline{\mathcal{M}}_{g, \alpha, \beta}\left(\mathbb{P}^{1}\right)$ be the moduli space of stable relative maps to $\mathbb{P}^{1}$ relative to two points 0 and $\infty$, where branching above 0 is given by a partition $\alpha \vdash d$ and the branching above $\infty$ is given by a partition $\beta \vdash d$. By the Riemann-Hurwitz formula, the expected number of branch points away from 0 and $\infty$ is

$$
r_{\alpha, \beta}^{g}:=l(\alpha)+l(\beta)+2 g-2,
$$

and the moduli space supports a virtual fundamental class

$$
\left[\overline{\mathcal{M}}_{g, \alpha, \beta}\left(\mathbb{P}^{1}\right)\right]^{\text {vir }} \in A_{r_{\alpha, \beta}^{g}}\left(\overline{\mathcal{M}}_{g, \alpha, \beta}\left(\mathbb{P}^{1}\right)\right)
$$

of this dimension. There is also a Fantechi-Pandharipande branch morphism

$$
b r: \overline{\mathcal{M}}_{g, \alpha, \beta}\left(\mathbb{P}^{1}\right) \rightarrow \operatorname{Sym}^{r_{\alpha, \beta}^{g}}\left(\mathbb{P}^{1}\right) .
$$

A mild variation of this is the case of relative stable maps to a non-rigidified target (see GV3, Sec. 2.4]; these are sometimes called "rubber maps"), where two relative maps to $\left(\mathbb{P}^{1}, 0, \infty\right)$ are considered the same if they differ by an automorphism of the target $\mathbb{P}^{1}$ preserving 0 and $\infty$ (an element of the group $\left.\mathbb{C}^{*}\right)$. This moduli space $\overline{\mathcal{M}}_{g, \alpha, \beta}\left(\mathbb{P}^{1}\right) \sim$ of such objects supports a virtual fundamental class of dimension one less than that of the "unrigidified" usual space:

$$
\left[\overline{\mathcal{M}}_{g, \alpha, \beta}\left(\mathbb{P}^{1}\right)_{\sim}\right]^{\text {vir }} \in A_{r_{\alpha, \beta}^{g}-1}\left(\overline{\mathcal{M}}_{g, \alpha, \beta}\left(\mathbb{P}^{1}\right)_{\sim}\right) .
$$

There is an obvious map $\phi: \overline{\mathcal{M}}_{g, \alpha, \beta}\left(\mathbb{P}^{1}\right) \rightarrow \overline{\mathcal{M}}_{g, \alpha, \beta}\left(\mathbb{P}^{1}\right)_{\sim}$ "forgetting the rigidification of the target". The two fundamental classes are related by the following result.

Proposition 3.1. GV3, Lem. 4.6]

$$
\phi_{*}\left(b r^{*}(L) \cap\left[\overline{\mathcal{M}}_{g, \alpha, \beta}\left(\mathbb{P}^{1}\right)\right]^{\mathrm{vir}}\right)=r_{\alpha, \beta}^{g}\left[\overline{\mathcal{M}}_{g, \alpha, \beta}\left(\mathbb{P}^{1}\right)_{\sim}\right]^{\mathrm{vir}}
$$

where $L$ is the class in $\operatorname{Sym}_{\alpha, \beta}^{r^{g}}\left(\mathbb{P}^{1}\right)$ corresponding to those $r_{\alpha, \beta}^{g}$-tuples of points containing a given fixed point $p_{0}$.

Intuitively, this is because given any unrigidified map in $\overline{\mathcal{M}}_{g, \alpha, \beta}\left(\mathbb{P}^{1}\right)_{\sim}$, there should be $r_{\alpha, \beta}^{g}$ ways to "rigidify" it so that a branch point maps to $1 \in \mathbb{P}^{1}$, as there "should be" $r_{\alpha, \beta}^{g}$ branch points away from 0 and $\infty$.

3.1.3. Relative stable maps with rational tails. We define the moduli spaces of relative stable maps with rational tails $\mathcal{M}_{g, \alpha}\left(\mathbb{P}^{1}\right)^{r t}$ as the analogous moduli spaces of maps where the source curve is a nodal curve with rational tails. This is an open substack of the space of relative stable maps. We define $\mathcal{M}_{g, \alpha, \beta}\left(\mathbb{P}^{1}\right)^{r t}$ similarly.

3.1.4. Relative stable maps with possibly disconnected source. The above definitions of relative maps would all make sense without the requirement that the source curve be connected. The resulting moduli space of maps from "possibly-disconnected" curves to $\mathbb{P}^{1}$ relative to one point is denoted by $\overline{\mathcal{M}}_{g, \alpha}\left(\mathbb{P}^{1}\right) \bullet$, and similarly for maps relative to two points. 
3.1.5. Observations using the Riemann-Hurwitz formula. We make some crucial observations, which are straightforward to verify using the Riemann-Hurwitz formula. We shall use them repeatedly. A degree $d$ cover is said to be completely branched over a point if the branching data is $(d) \vdash d$. Define a trivial cover of $\mathbb{P}^{1}$ to be a map $\mathbb{P}^{1} \rightarrow \mathbb{P}^{1}$ of the form $[x ; y] \mapsto\left[x^{u} ; y^{u}\right]$, branched only over 0 and $\infty$.

Remark 3.2. Suppose we have a map $C \rightarrow \mathbb{P}^{1}$ from a nodal (possibly disconnected) curve, unbranched away from 0 and $\infty$, where $C$ is non-singular over 0 and $\infty$. Then it is a disjoint union of trivial covers.

Remark 3.3. a) Suppose we have a map from a nodal curve $C$ to $\mathbb{P}^{1}$, with no branching away from 0,1 , and $\infty$, simple branching at 1 , and non-singular over 0 and $\infty$. Then it is a union of trivial covers, with one further component, that is non-singular of genus 0 , completely branched over one of $\{0, \infty\}$, and with two preimages over the other (see Figure 3). We call this an almost-trivial cover.

b) More generally, suppose we have a map from a curve $C$ to a chain of $\mathbb{P}^{1}$ 's, satisfying the kissing or predeformability condition, unbranched except for two non-singular points 0 and $\infty$ on the ends of the chain, and simple branching at one more point. Then the map looks like a number of trivial covers glued together, with one further almost-trivial cover $\mathbb{P}^{1} \rightarrow \mathbb{P}^{1}$ of the sort described in Remark 3.3(a).

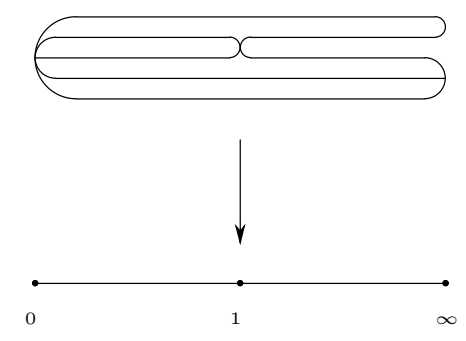

FiguRE 3. Almost-trivial covers: All branched covers from non-singular curves with simple branching above 1 and no other branching away from 0 and $\infty$ must look like this.

Remark 3.4. a) If we have a map from a nodal curve $C$ to $\mathbb{P}^{1}$, with total branching away from 0 and $\infty$ of degree less than $2 g$, and non-singular over 0 and $\infty$, then $C$ has no components of geometric genus $g$. If instead the total branching away from 0 and $\infty$ is precisely $2 g$, and $C$ has a component of geometric genus $g$, then the cover is a disjoint union of trivial covers, and one connected curve $C^{\prime}$ of arithmetic genus $g$, where the map $C^{\prime} \rightarrow \mathbb{P}^{1}$ is completely branched over 0 and $\infty$.

b) Suppose more generally we have a map from a curve $C$ to a chain of $\mathbb{P}^{1}$ 's, satisfying the kissing condition, with branching of less than $2 g$ away from the nodes and two smooth points 0 and $\infty$ on the ends of the chain, then $C$ has no component of geometric genus $g$. If the branching is precisely $2 g$ away from the nodes and 0 and $\infty$, then the map is a number of trivial covers glued together, plus one other cover of the sort described in Remark 3.4)(a).

The following fact will prove essential.

Theorem 3.5. Let $\mathbb{G}_{g, d, \sim}:=\mu_{*}\left[\mathcal{M}_{g,(d),(d)}\left(\mathbb{P}^{1}, d\right)_{\sim}^{r t}\right]^{\mathrm{vir}}$ where $\mu$ is the moduli map to $\mathcal{M}_{g, 2}^{r t}$, remembering the curve and the points over 0 and $\infty$. Then $\mathbb{G}_{g, d, \sim}=d^{2 g} \mathbb{G}_{g, 1, \sim}$. Similarly, if $\mathbb{G}_{g, d}:=\mu_{*}\left[b r^{*}(L) \cap \mathcal{M}_{g,(d),(d)}\left(\mathbb{P}^{1}, d\right)^{r t}\right]^{\mathrm{vir}}$, then $\mathbb{G}_{g, d}=d^{2 g} \mathbb{G}_{g, 1}$.

Proof. The second statement follows from the first by Proposition 3.1. We now prove the first. 
Let $\nu:$ Jac $\rightarrow \mathcal{M}_{g, 2}^{r t}$ be the universal Jacobian or Picard stack over $\mathcal{M}_{g, 2}^{r t}$. Let Jac $[d]:=$ $\operatorname{ker}(\mathrm{Jac} \stackrel{\times d}{\longrightarrow} \mathrm{Jac})$ be the $d$-torsion substack. (Points of Jac $[d]$ correspond to curves along with a $d$-torsion point.)

Let $p$ and $q$ be the names of the points of the genus $g$ curve parametrized by $\mathcal{M}_{g, 2}^{r t}$. Let $s_{p-q}$ : $\mathcal{M}_{g, 2}^{r t} \rightarrow$ Jac be the section corresponding to the line bundle $\mathcal{O}(p-q)$. There is a natural (stacktheoretic) isomorphism

$$
\mathcal{M}_{g,(d),(d)}\left(\mathbb{P}^{1}\right)_{\sim}^{r t} \cong \operatorname{Jac}[d] \cap s_{p-q}\left(\mathcal{M}_{g, 2}^{r t}\right),
$$

as follows. Given any family of such relative stable maps (with rational tails), let $p$ and $q$ be the preimage of 0 and $\infty$ respectively. Note that the target $\mathbb{P}^{1}$ never degenerates (or sprouts) in such a family. Thus $\mathcal{O}(d p-d q) \cong \mathcal{O}$, and hence $\mathcal{O}(p-q)$ is indeed $d$-torsion. Conversely, given any family of curves in $\mathcal{M}_{g, 2}^{r t}$ where $\mathcal{O}(p-q)$ is $d$-torsion, we obtain a unique family of relative stable maps with unrigidified target of the desired sort. (It is essential to note that this isomorphism holds over the boundary of $\mathcal{M}_{g, 2}^{r t}$ as well.)

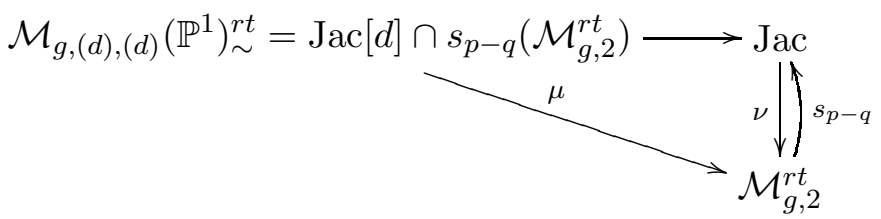

Both sides of the isomorphism (77) have natural virtual fundamental classes, the latter by intersecting the section $s_{p-q}\left(\mathcal{M}_{g, 2}^{r t}\right)$ with the local complete intersection Jac $[d] \hookrightarrow$ Jac. We claim that these virtual fundamental classes agree. (It is easy to show that away from the boundary of $\mathcal{M}_{g, 2}^{r t}$, both will agree with the actual fundamental class, but we shall not need this fact.) We show this by showing that they have the same deformation-obstruction theory over $\mathcal{M}_{g, 2}^{r t}$.

We use the description of the deformation-obstruction theory of $\overline{\mathcal{M}}_{g,(d),(d)}\left(\mathbb{P}^{1}\right)$ over $\overline{\mathcal{M}}_{g, 2}$ given in [GV3, Sec. 2.8]. On the locus, at a relative stable map $\left[f: C \rightarrow \mathbb{P}^{1}\right]$, the relative deformation space is identified with $H^{0}\left(C, f^{*} T_{\mathbb{P}^{1}}(-[0]-[\infty])\right)=H^{0}\left(C, f^{*} \mathcal{O}_{\mathbb{P}^{1}}\right)$ in [GV3, equ. (1)]. (The formula given in [GV3] is more involved, as it applies in more general situations. In particular, in the locus, the target never sprouts, so the notation $f^{\dagger}$ in [GV3] agrees with $f^{*}$. Also $T_{\mathbb{P}^{1}}(-\log [0]-\log [\infty])=$ $T_{\mathbb{P}^{1}}(-[0]-[\infty])$, as $\mathbb{P}^{1}$ has dimension 1.) Thus the relative deformation space is canonically identified with $H^{0}\left(C, \mathcal{O}_{C}\right)$, which is one-dimensional. But such deformations correspond precisely to the $\mathbb{C}^{*}$-action induced by its action on the target (keeping the map otherwise fixed), so the relative deformation space of maps to $\mathbb{P}^{1}$ with unrigidified target is 0 .

The relative obstruction space $\operatorname{RelOb}(f)$ at a relative stable map $\left[f: C \rightarrow \mathbb{P}^{1}\right]$ has a natural filtration [GV3, equ. (2)]

$$
0 \rightarrow H^{1}\left(C, f^{*} T_{\mathbb{P}^{1}}(-[0]-[\infty])\right) \rightarrow \operatorname{RelOb}(f) \rightarrow H^{0}\left(C, f^{-1} \mathcal{E} x t^{1}\left(\Omega_{\mathbb{P}^{1}}(-[0]-[\infty]), \mathcal{O}_{\mathbb{P}^{1}}\right)\right) \rightarrow 0 .
$$

Now $\mathcal{E} x t^{1}\left(\mathcal{O}_{\mathbb{P}^{1}}, \mathcal{O}_{\mathbb{P}^{1}}\right)=0$, so this filtration gives a natural isomorphism

$$
H^{1}\left(C, \mathcal{O}_{C}\right) \stackrel{\sim}{\longrightarrow} \operatorname{RelOb}(f) .
$$

But this is the relative obstruction space of the intersection: the normal bundle to the $d$-torsion locus Jac $[d]$ is canonically $H^{1}\left(C, \mathcal{O}_{C}\right)$. Thus we have shown that the isomorphism (7) indeed extends to an isomorphism of virtual fundamental classes.

We conclude by noting that $[\mathrm{Jac}[d]]=d^{2 g}[\mathrm{Jac}[1]]$ in $A_{*}(\mathrm{Jac})$. This follows from [DM, Sec. 2.15], as observed in the proof of [Lo, Lem. 2.10].

Remark. $\mathbb{G}_{g, 1}$ will be our "natural generator" of $R_{2 g-1}\left(\mathcal{M}_{g, 1}\right)$. The generator used by Looijenga in [Lo] is the hyperelliptic locus $\mathbb{H} y p$. They are related as follows: $(2 g+2)(2 g+1) \mathbb{H} y p=\left(2^{2 g}-\right.$ 
1) $\mathbb{G}_{g, 1, \sim}$ (both count hyperelliptic covers with two fixed branch points), and $\mathbb{G}_{g, 1}=2 g \mathbb{G}_{g, 1, \sim}$ from Proposition 3.1, see Corollary 6.5 and its proof.

3.2. Faber-Hurwitz classes. We define Hurwitz classes, following GV3]. Our motivation is as follows. Suppose we are interested in dimension $j$ classes on $\overline{\mathcal{M}}_{g, n}$. One way of producing a family of genus $g$ curves with $n$-marked points is by considering branched covers of $\mathbb{P}^{1}$ with fixed branching over $\infty$ corresponding to a partition $\left(\alpha_{1}, \ldots, \alpha_{n}\right)$ of $d$ with $n$ parts. The curve in question will be the source of the map to $\mathbb{P}^{1}$, and the $n$ points will be the points over $\infty$. This Hurwitz scheme (the moduli space of such maps) will have dimension $r_{\alpha}^{g}$. In order to get a class of dimension $j$, we fix all but $j$ branch points. We can then push forward this class to $\overline{\mathcal{M}}_{g, n}$. The definition of "Hurwitz class" involves doing this "virtually":

$$
\mathbb{H}_{j}^{g, \alpha}:=\mu_{*}\left(b r^{*}(L)^{r_{\alpha}^{g}-j} \cap\left[\overline{\mathcal{M}}_{g, \alpha}\left(\mathbb{P}^{1}\right)\right]^{\mathrm{vir}}\right)
$$

where $\mu$ is the forgetful map $\overline{\mathcal{M}}_{g, \alpha}\left(\mathbb{P}^{1}\right) \rightarrow \overline{\mathcal{M}}_{g, n}$ and $b r$ is the branch morphism (5). Here, as in Proposition 3.1, $L$ is the class in $\operatorname{Sym}^{r_{\alpha}^{g}}\left(\mathbb{P}^{1}\right)$ corresponding to unordered tuples of points containing a given fixed point $p_{0}$.

Define the Faber-Hurwitz class $\mathbb{F}^{g, \alpha}$ by

$$
\mathbb{F}^{g, \alpha}:=\left.\mathbb{H}^{g, \alpha}\right|_{\mathcal{M}_{g, n}^{r t}} \in A_{2 g-1}\left(\mathcal{M}_{g, n}^{r t}\right) .
$$

Equivalently, we can consider the space of relative stable maps with rational tails $\mathcal{M}_{g, \alpha}\left(\mathbb{P}^{1}\right)^{r t}$, along with its virtual fundamental class; fix all but $2 g-1$ branch points; and push forward to the moduli space $\mathcal{M}_{g, n}^{r t}$. Let

$$
r_{g, \alpha}^{\mathrm{Fab}}:=r_{\alpha}^{g}-(2 g-1)=d+l(\alpha)-1
$$

be the number of fixed branch points on $\mathbb{P}^{1}$ in this construction.

We shall now understand this class in two ways, by degeneration and localization. The first will connect us to a Hurwitz-type problem (and join-cut type recursion) that we can solve. The second will connect us to the tautological ring.

Readers less comfortable with these ideas from Gromov-Witten theory may go directly to the recursions that are obtained by these means without losing the thread of the paper. These recursions are treated quite cleanly through transforms of the corresponding partial differential equations (see Section 4).

3.3. Degeneration of Faber-Hurwitz classes. We now describe a join-cut type recursive formula for the Faber-Hurwitz classes $\mathbb{F}^{g, \alpha}$. This will allow us to compute $\mathbb{F}^{g, \alpha}$ in terms of the putative generator $\mathbb{G}_{g, 1}$, first recursively, and later, in closed form. We use Jun Li's degeneration formula [Li1, Li2, which in our case states that, if we degenerate $\left[\overline{\mathcal{M}}_{g, \alpha}\left(\mathbb{P}^{1}\right) \bullet\right]^{\text {vir }}$ by degenerating the target into two components $\mathbb{P}_{L}^{1} \cup \mathbb{P}_{R}^{1}$, where the point corresponding to $\alpha$ is on the right component $\mathbb{P}_{R}^{1}$, then

$$
\left[\overline{\mathcal{M}}_{g, \alpha}\left(\mathbb{P}^{1}\right) \bullet\right]^{\mathrm{vir}}=\sum_{g_{1}, g_{2}, \gamma}\left(\prod_{i} \gamma_{i}\right)\left[\overline{\mathcal{M}}_{g_{1}, \gamma}\left(\mathbb{P}^{1}\right) \bullet\right]^{\mathrm{vir}} \otimes\left[\overline{\mathcal{M}}_{g_{2}, \gamma, \alpha}\left(\mathbb{P}^{1}\right) \bullet\right]^{\mathrm{vir}}
$$

where the sum is over all "splitting of the data": $|\gamma|=|\alpha|, g_{1}+g_{2}+l(\gamma)-1=g$, and the genus $g_{1}$ cover $C_{1}$ is glued to the genus $g_{2}$ cover $C_{2}$ over the node $\mathbb{P}_{L}^{1} \cap \mathbb{P}_{R}^{1}$ by gluing the point corresponding to $\gamma_{i}$ on $C_{1}$ to the point corresponding to $\gamma_{i}$ on $C_{2}$. There is the obvious variation for $\left[\overline{\mathcal{M}}_{g, \alpha, \beta}\left(\mathbb{P}^{1}\right) .\right]^{\text {vir }}$ for stable maps relative to two points. If we are interested in spaces with connected source, i.e. $\overline{\mathcal{M}}_{g, \alpha}\left(\mathbb{P}^{1}\right)$ rather than $\overline{\mathcal{M}}_{g, \alpha}\left(\mathbb{P}^{1}\right)$ •, then we include only the summands where the union $C_{1} \cup C_{2}$ is connected. We shall "cap" these equalities with pullbacks under the branch morphism, 
and interpret them as considering maps with given branch points, where we shall specify how many branch points degenerate to $\mathbb{P}_{L}^{1}$ and $\mathbb{P}_{R}^{1}$, respectively.

Lemma 3.6. If $j<2 g-1$, then the restriction of $\mu_{*}\left(\mathbb{H}_{j}^{g, \alpha}\right)$ to the rational-tails locus $\mathcal{M}_{g, n}^{r t}$ is 0 .

Proof. Degenerate the target $\mathbb{P}^{1}$ into a chain of $\left(r_{\alpha}^{g}-j\right) \mathbb{P}^{1}$ 's, where each of the $r_{\alpha}^{g}-j$ fixed branch points lies in a different component of the target. Then by the degeneration formula, this class will be obtained as a sum (over all choices of splittings of the data) of classes glued together from virtual fundamental classes of various spaces of relative stable maps to each component. But any such relative stable map to one component, with points 0 and $\infty$ connecting it to adjacent elements of the chain (with the obvious variation if it is the end of the chain) has at most $j+1<2 g$ branch points away from 0 and $\infty$. Hence by Remark 3.4, there is no curve of geometric genus $g$ mapping to this component. Thus, after degeneration, the source curve can have no irreducible component of genus $g$, and hence the class is 0 in $\mathcal{M}_{g, n}^{r t}$.

Theorem 3.7 (Degeneration Theorem - Join-cut recursion). If $r_{g, \alpha}^{\mathrm{Fab}}>0$, then

$$
\mathbb{F}^{g, \alpha}=\sum_{i+j=\alpha_{k}} i j H_{\alpha^{\prime}}^{0} \mathbb{F}^{g, \alpha^{\prime \prime}}\left(\begin{array}{c}
r_{g, \alpha}^{\mathrm{Fab}}-1 \\
r_{g, \alpha^{\prime \prime}}^{\mathrm{Fab}}
\end{array}\right)+\sum_{\alpha_{i}, \alpha_{j}}\left(\alpha_{i}+\alpha_{j}\right) \mathbb{F}^{g, \alpha^{\prime}}+\sum_{i=1}^{l(\alpha)} \alpha_{i}^{2 g+1} H_{\alpha}^{0} \mathbb{G}_{g, 1} .
$$

where $\alpha^{\prime}, \alpha^{\prime \prime}$, etc., are as defined in the proof below.

This recursion inductively determines $\mathbb{F}^{g, \alpha}$ in terms of $\mathbb{G}_{g, 1}$ (Theorem 3.8). There is no "base case" necessary. This result is best stated in terms of generating series (see Corollary [3.9).

Proof. We obtain a recursion for Faber-Hurwitz classes by a similar argument to that used in Lemma 3.6 above. We degenerate the target into two pieces $\mathbb{P}_{L}^{1} \cup \mathbb{P}_{R}^{1}$, where $\infty$ and one of the $r_{g, \alpha}^{\mathrm{Fab}}=r_{\alpha}^{g}-(2 g-1)=d+l(\alpha)-1$ fixed branch points are on the right component $\mathbb{P}_{R}^{1}$, and the remaining $r_{g, \alpha}^{\mathrm{Fab}}-1$ fixed branch points are on the left component $\mathbb{P}_{L}^{1}$. By the degeneration formula (10), this class will be obtained as a sum (over all choices of splittings of the data) of classes glued together from virtual fundamental classes of various spaces of relative stable maps to each component. We discard every term that does not have a smooth component of genus $g$.

There are two cases: the genus $g$ curve maps to the left component $\mathbb{P}_{L}^{1}$ or the right component $\mathbb{P}_{R}^{1}$.

Case 1: If the genus $g$ curve maps to the right component $\mathbb{P}_{R}^{1}$, then by Remark 3.4, all the moving branch points must also map to $\mathbb{P}_{R}^{1}$ (we need $2 g$ branching total away from $\mathbb{P}_{L}^{1} \cap \mathbb{P}_{R}^{1}$ and $\infty$ in order to have a genus $g$ curve in the cover). Also by Remark $\left[3.4\right.$ (a), the cover of $\mathbb{P}_{R}^{1}$ must be a union of trivial covers, together with one cover by a connected arithmetic genus $g$ curve that is completely branched over $\infty$ and $\mathbb{P}_{L}^{1} \cap \mathbb{P}_{R}^{1}$. In particular, the partition over the node $\mathbb{P}_{L}^{1} \cap \mathbb{P}_{R}^{1}$ must be the same as the partition $\alpha$ over $\infty$, and the genus $g$ component can be on the component of the source corresponding to any $\alpha_{j}$. Also, the cover of $\mathbb{P}_{L}^{1}$ must be genus 0 and connected, and the number of such covers is the genus 0 Hurwitz number

$$
H_{\alpha}^{0}=(d-2+l(\alpha)) ! d^{l(\alpha)-3} \prod_{i=1}^{l(\alpha)} \frac{\alpha_{i}^{\alpha_{i}}}{\alpha_{i} !} .
$$

(This celebrated formula was first given by Hurwitz, and has now been proved in many ways, see for example GJ2.) This case is depicted pictorially in Figure 4, here the genus $g$ curve corresponds to $\alpha_{1}$. We thus get a contribution to $\mathbb{F}^{g, d}$ of

$$
\sum_{i=1}^{l(\alpha)} \alpha_{i} H_{\alpha}^{0} \mathbb{G}_{g, \alpha_{i}}=\sum_{i=1}^{l(\alpha)} \alpha_{i}^{2 g+1} H_{\alpha}^{0} \mathbb{G}_{g, 1} .
$$


The equality in (12) is through Theorem 3.5. The contributions to the left side of (12) are as follows: we have a contribution of $\mathbb{G}_{g, \alpha_{i}}$ from the map to $\mathbb{P}_{L}^{1}$. We have a contribution of $\mathbb{G}_{g, \alpha_{i}} \prod_{j \neq i}\left(1 / \alpha_{j}\right)$ from the map to $\mathbb{P}_{R}^{1}$ (since the trivial cover contributes the order $\alpha_{k}$ of the automorphism group and we are therefore counting objects weighted by $1 / \alpha_{k}$.). We have a multiplicity of $\prod \alpha_{i}$ from the kissing condition.

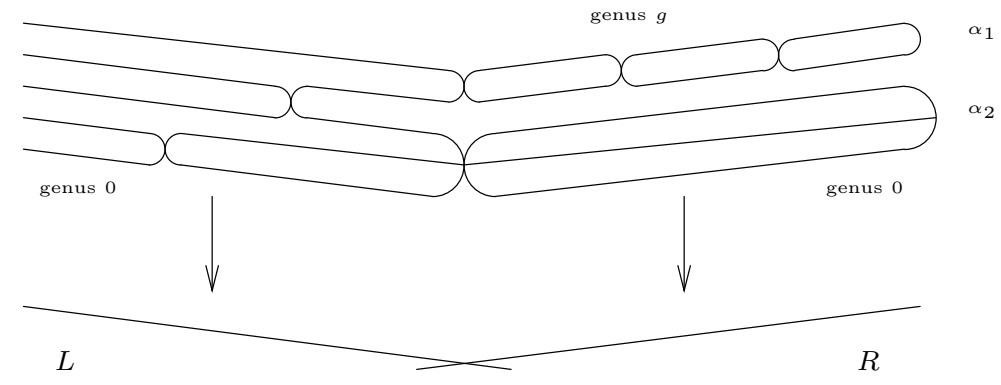

FiguRE 4. First case in degeneration argument in proof of Theorem 3.7

Case 2a: If otherwise the genus $g$ curve maps to the left component $\mathbb{P}_{L}^{1}$, then by Lemma 3.6, all the non-fixed branch points must also map to $\mathbb{P}_{L}^{1}$ (as we need at least $2 g-1$ moving branch points in order to get a non-zero contribution), so the only branching over $\mathbb{P}_{R}^{1}$ away from its 0 and $\infty$ is simple branching over one (fixed) point. By Remark 3.3 , the cover of $\mathbb{P}_{R}^{1}$ is a union of trivial covers, with one almost-trivial cover. If the almost-trivial cover of $\mathbb{P}_{R}^{1}$ is completely branched over $\mathbb{P}_{L}^{1} \cap \mathbb{P}_{R}^{1}$, and has two preimages over $\infty$ (e.g. as shown in Figure 3i), then it can connect any two of the points over $\infty$ (corresponding to two parts of the partition $\alpha$, say $\alpha_{i}$ and $\alpha_{j}$ ). The virtual fundamental class of this space of relative stable maps to $\mathbb{P}_{R}^{1}$ is then $\alpha_{i} \alpha_{j} / \prod \alpha_{i}$ : any trivial cover corresponding to $\alpha_{k}(k \neq i, j)$ is weighted by $1 / \alpha_{k}$. The space of relative maps to $\mathbb{P}_{L}^{1}$ corresponds to maps from a connected curve of genus $g$ curve, with branching over $\mathbb{P}_{L}^{1} \cap \mathbb{P}_{R}^{1}$ corresponding to the partition $\alpha^{\prime}$ obtained by removing $\alpha_{i}$ and $\alpha_{j}$ from $\alpha$, and adding $\alpha_{i}+\alpha_{j}$, with all but $2 g-1$ branch points fixed. In other words, the contribution is precisely $\mathbb{F}^{g, \alpha^{\prime}}$. Finally, the multiplicity in the degeneration formula is the product of the multiplicities of the kissing over the node $\mathbb{P}_{L}^{1} \cap \mathbb{P}_{R}^{1}$, i.e. $\prod \alpha_{i}^{\prime}=\left(\prod \alpha_{i}\right)\left(\alpha_{i}+\alpha_{j}\right) /\left(\alpha_{i} \alpha_{j}\right)$. Thus we obtain a contribution to $\mathbb{F}^{g, \alpha}$ of

$$
\sum_{\alpha_{i}, \alpha_{j}}\left(\alpha_{i}+\alpha_{j}\right) \mathbb{F}^{g, \alpha^{\prime}}
$$

Case 2b: Finally, if the almost-trivial cover over $\mathbb{P}_{R}^{1}$ has one preimage over $\infty$ (corresponding to $\alpha_{k}$, say), and two preimages over $\mathbb{P}_{L}^{1} \cap \mathbb{P}_{R}^{1}\left(q_{1}\right.$ and $q_{2}$, say, branching with multiplicity $i$ and $j$ respectively, where $i+j=\alpha_{k}$ ), then we have contributions of $\alpha_{k} / \prod \alpha_{i}$ from the cover of $\mathbb{P}_{R}^{1}$, and $\prod \alpha_{i} \times i j / \alpha_{k}$ from the kissing multiplicities. We next examine the contribution from the stable relative maps to $\mathbb{P}_{L}^{1}$. The cover must have two connected components, one containing the point $q_{1}$ and one containing the point $q_{2}$. As the map has a component of geometric genus $g$, one of these two components must be arithmetic genus $g$, and the other must be arithmetic genus 0 . Suppose the genus 0 curve contains to $q_{1}$, and the genus $g$ curve contains $q_{2}$. Suppose the partition corresponding to the genus 0 curve is $\alpha^{\prime}$ (i.e. its ramification above the node $\mathbb{P}_{L}^{1} \cap \mathbb{P}_{R}^{1}$ ), and the partition corresponding to the genus $g$ curve is $\alpha^{\prime \prime}$, so $\alpha^{\prime}+\alpha^{\prime \prime}=\alpha-\alpha_{k}+\{i, j\}$, and $i \in \alpha^{\prime}$, $j \in \alpha^{\prime \prime}$. In order for the contribution to be non-zero, by Lemma 3.6, all of the $(2 g-1)$ "moving" branching must belong to the genus $g$ component, so all of the genus 0 curve's branch points must be fixed. There are $\left(\begin{array}{c}r_{g, \alpha}^{\mathrm{Fab}}-1 \\ r_{g, \alpha^{\prime \prime}}^{\mathrm{Fab}}\end{array}\right)$ ways of choosing which fixed branch point on $\mathbb{P}_{L}^{1}$ belongs to the genus 0 curve, and which belongs to the genus $g$ curve. Hence the contribution of the cover of $\mathbb{P}_{L}^{1}$ is 
$H_{\alpha^{\prime}}^{0} \mathbb{F}^{g, \alpha}\left(\begin{array}{c}r_{g, \alpha}^{\mathrm{Fab}}-1 \\ r_{g, \alpha^{\prime \prime}}^{\mathrm{Fab}}\end{array}\right)$, so the combined contribution to $\mathbb{F}^{g, \alpha}$ from this case is

$$
\sum_{i+j=\alpha_{k}} i j H_{\alpha^{\prime}}^{0} \mathbb{F}^{g, \alpha^{\prime \prime}}\left(\begin{array}{c}
r_{g, \alpha}^{\mathrm{Fab}}-1 \\
r_{g, \alpha^{\prime \prime}}^{\mathrm{Fab}}
\end{array}\right) .
$$

Summing the three types of contribution, we obtain the result.

Theorem 3.8. For each fixed $g, \mathbb{F}^{g, \alpha}$ is a rational multiple of $\mathbb{G}_{g, 1}$, as determined by the recursion of Theorem 3.7.

Proof. By Remark 3.4(a), if $r_{g, \alpha}^{\mathrm{Fab}}=0$, then $\mathbb{F}^{g, \alpha}=0$; this is the trivial base case of the recursion in Theorem 3.7. The result follows by induction.

For convenience, we define the Faber-Hurwitz number $F_{\alpha}^{g} \in \mathbb{Q}$ to be this multiple of $\mathbb{G}_{g, 1}$, to remind us that these classes are all commensurate:

$$
F_{\alpha}^{g} \mathbb{G}_{g, 1}:=\mathbb{F}^{g, \alpha} .
$$

(Corollary 6.6 is a sign that $F_{\alpha}^{g}$ is a well-behaved quantity to consider.)

Theorem 3.7 is best formulated in terms of generating series. A natural generating series for genus 0 Hurwitz numbers is

$$
\widehat{H}^{0}(z ; \mathbf{p}):=\sum_{\alpha \in \mathcal{P}} z^{|\alpha|} \frac{p_{\alpha}}{|\operatorname{Aut} \alpha|} \frac{H_{\alpha}^{0}}{r_{\alpha}^{0} !},
$$

where $\mathbf{p}=\left(p_{1}, p_{2}, \ldots\right)$, and a natural generating series for Faber-Hurwitz numbers, which we shall call the Faber-Hurwitz series, is

$$
F^{g}(z ; \mathbf{p}):=\sum_{\alpha \in \mathcal{P}} z^{|\alpha|} \frac{p_{\alpha}}{\mid \text { Aut } \alpha \mid} \frac{F_{\alpha}^{g}}{r_{g, \alpha}^{\mathrm{Fab} !}} .
$$

Then the recursion of Theorem 3.7, for classes, becomes the following (linear) partial differential equation for $F^{g}$.

Corollary 3.9 (Degeneration Theorem - Join-cut Equation for Faber-Hurwitz Series). For $g \geq 1$, $F^{g}(z ; \mathbf{p})$ is the unique formal power series solution to

$$
\begin{aligned}
\left(z \frac{\partial}{\partial z}-1+\sum_{i \geq 1} p_{i} \frac{\partial}{\partial p_{i}}\right) F^{g}= & \sum_{i, j \geq 1} p_{i+j}\left(i \frac{\partial}{\partial p_{i}} \widehat{H}^{0}\right)\left(j \frac{\partial}{\partial p_{j}} F^{g}\right) \\
& +\frac{1}{2} \sum_{i, j \geq 1} p_{i} p_{j}(i+j) \frac{\partial}{\partial p_{i+j}} F^{g}+\sum_{i \geq 1} i^{2 g+1} p_{i} \frac{\partial}{\partial p_{i}} \widehat{H}^{0},
\end{aligned}
$$

In Corollary [3.9, we have changed from classes to numbers by erasing $\mathbb{G}_{g, 1}$ from Theorem 3.7 via (13). To obtain a generating series for Faber-Hurwitz classes, we would simply multiply this series by $\mathbb{G}_{g, 1}$.

3.4. Localization of Faber-Hurwitz classes. This section contains the Localization Tree Theorem for Faber-Hurwitz classes. It is to be thought of in conjunction with the Degeneration Theorem. The theorem gives the fundamental relationship between Faber-Hurwitz classes on the one hand, and intersection numbers and (genus 0) single and double Hurwitz numbers on the other. Implicitly, it allows us to determine the Faber-Hurwitz numbers. It involves a sum over a set $\mathcal{T}_{g, m}$, which is one of three sets of trees defined as follows. 
Definition 3.10 (Localization trees). Consider rooted trees with the following properties. For each tree $\mathfrak{t}$ there are three classes of vertices: $\mathcal{V}_{0}(\mathfrak{t})$, the 0 -vertices; $\mathcal{V}_{\infty}(\mathfrak{t})$, the $\infty$-vertices; $\mathcal{V}_{t}(\mathfrak{t})$, the $t$ vertices. All t-vertices are monovalent (here $t$ stands for "tail"). The number of non-root 0 -vertices in $\mathfrak{t}$ is denoted by $\eta_{0}(\mathfrak{t})$. The non-root 0 -vertices are labelled (each receives one of $\eta_{0}(\mathfrak{t})$ labels in all possible ways). The $\infty$-vertices are not labelled. There are two classes of edges: $\mathcal{E}_{0 \infty}(\mathfrak{t})$, the $0 \infty$-edges, each of which joins a 0 -vertex to an $\infty$-vertex; $\mathcal{E}_{\infty t}(\mathfrak{t})$, the $\infty$-edges, each of which joins a non-root $\infty$-vertex to a t-vertex.

There is at least one edge. Each edge is assigned a positive integer weight, and the integer weight on an $0 \infty$-edge $e$ is denoted by $\epsilon(e)$. The t-vertices incident with an edge of weight $k$ are labelled among themselves, for each $k \geq 1$. The list of the weights on all $0 \infty$-edges incident with a 0 -vertex $v$ is specified by the partition $\delta^{v}(\mathfrak{t})$, the list of the weights on all $0 \infty$-edges incident with an $\infty$ vertex $v$ is specified by the partition $\beta^{v}(\mathfrak{t})$, and the list of the weights on all $\infty$ t-edges incident with a non-root $\infty$-vertex $v$ is specified by the partition $\gamma^{v}(\mathfrak{t})$. For each non-root $\infty$-vertex $v$, we impose the condition that

$$
\left|\beta^{v}(\mathfrak{t})\right|=\left|\gamma^{v}(\mathfrak{t})\right|
$$

The root-vertex may be either a 0 -vertex or an $\infty$-vertex, and is denoted by $\bullet$. For $m \geq 1$, we define $\mathcal{T}_{g, m}$ to be the set of rooted trees above in which the root-vertex is a 0 -vertex of degree $m$. For $j \geq 1$, we define $\mathcal{T}_{0, j}$ to be the subset of $\mathcal{T}_{g, 1}$ in which the edge incident with the root-vertex has weight $j$. For $j \geq 1$, we define $\mathcal{T}_{\infty, j}$ to be the set of rooted trees above in which the root-vertex is a monovalent $\infty$-vertex, and the edge incident with the root-vertex has weight $j$.

We refer to the trees in any of the sets $\mathcal{T}_{g, m}, \mathcal{T}_{0, j}$ and $\mathcal{T}_{\infty, j}$ as "localization trees".

Hereinafter for any localization tree $\mathfrak{t}$ we shall subsume the dependence on $\mathfrak{t}$ of the above sets of vertices and edges, and the partitions, by suppressing the occurrence of $t$ as an argument. We also define some more notation. Let

$$
r_{\infty}:=\sum_{v \in \mathcal{V}_{\infty}} r_{\gamma^{v}, \beta^{v}}^{0}
$$

and let

$$
\alpha:=\coprod_{v \in \mathcal{V}_{\infty}} \gamma^{v}
$$

be the partition formed by all the parts of each of the $\gamma^{v}$ 's for non-root $\infty$-vertices $v$, and let $d=|\alpha|$. Examples of such trees without a specified root are given in Figure 5 (these examples correspond to the geometric picture of Figure [6).
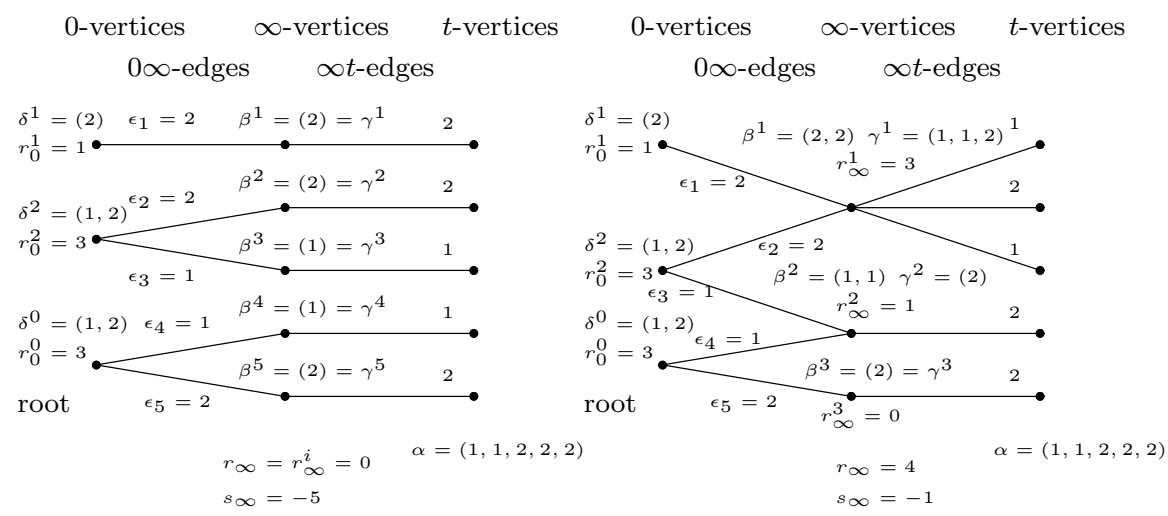

Figure 5. Sample decorated trees (Def. 3.10) corresponding roughly to the examples of Figure 6. Omitted are further combinatorial labelling of vertices. 
We now define some terminology that will allow us to write the relative virtual localization calculation cleanly. Let $\mathbb{P}_{m}^{g}\left(\alpha_{1}, \ldots, \alpha_{m}\right)$ be the dimension $2 g-1$ portion of

$$
\frac{1-\lambda_{1}+\cdots+(-1)^{g} \lambda_{g}}{\left(1-\alpha_{1} \psi_{1}\right) \cdots\left(1-\alpha_{m} \psi_{m}\right)}
$$

on $\mathcal{M}_{g, m}^{r t}$, viz.,

$$
\mathbb{P}_{m}^{g}\left(\alpha_{1}, \ldots, \alpha_{m}\right):=\sum_{\substack{a_{1}, \ldots, a_{m}, k \geq 0, a_{1}+\cdots+a_{m}+k=g-2+m}}(-1)^{k}\left\langle\tau_{a_{1}} \cdots \tau_{a_{m}} \lambda_{k}\right\rangle_{g}^{\mathbb{F a b}} \alpha_{1}^{a_{1}} \cdots \alpha_{m}^{a_{m}} .
$$

Note that $\mathbb{P}_{m}^{g}$ is a (Chow-valued) polynomial in the numbers $\alpha_{1}, \ldots, \alpha_{m}$, symmetric of degree between $m-2$ and $g-2+m$, and its leading coefficients (the portion of homogeneous degree $g-2+m$ ) are precisely the subject of Faber's Intersection Number Conjecture. (It is easy to show that the homogeneous degree $m-2$ and $m-1$ portions of this polynomial vanish, but we shall not need this fact.) We shall refer to $\mathbb{P}_{m}^{g}$ as the Faber polynomial.

For any localization tree $\mathfrak{t}$, let

$$
B(\mathfrak{t}):=\prod_{e \in \mathcal{E}_{0 \infty}} \epsilon(e), \quad C(\mathfrak{t}):=\prod_{v \in \mathcal{V}_{0}} \frac{H_{\delta^{v}}^{0}}{r_{\delta^{v}}^{0} !}, \quad D(\mathfrak{t}):=\prod_{v \in \mathcal{V}_{\infty}} \frac{H_{\gamma^{v}, \beta^{v}}^{0}}{r_{\gamma^{v}, \beta^{v}}^{0} !} .
$$

and, for $\mathfrak{t} \in \mathcal{T}_{g, m}$, let

$$
A(\mathfrak{t}):=\mathbb{P}_{m}^{g}\left(\delta^{\bullet}\right) \prod \frac{\epsilon(e)^{\epsilon(e)}}{\epsilon(e) !}
$$

where the product is over all edges $e$ incident with the root-vertex $\bullet$ of $\mathfrak{t}$. Let “ $\ddagger$ " as a superscript on a product denote the removal of the contribution of the root-vertex from that product.

Theorem 3.11 (Localization Tree Theorem - tree summation). For $g \geq 1$ and $\alpha=\left(\alpha_{1}, \ldots, \alpha_{m}\right) \vdash$ $d$

$$
\mathbb{F}^{g, \alpha}=\sum_{m \geq 1} \sum_{\mathfrak{t} \in \mathcal{T}_{g, m}}(-1)^{r_{\infty}} r_{g, \alpha}^{\mathrm{Fab} !}\left(\begin{array}{c}
r_{\alpha}^{g}-r_{\infty} \\
r_{g, \alpha}^{\mathrm{Fab}}
\end{array}\right) \frac{1}{\eta_{0}(\mathfrak{t}) !} A(\mathfrak{t}) B(\mathfrak{t}) C^{\ddagger}(\mathfrak{t}) D(\mathfrak{t})
$$

where the sum is subject to (18).

Note that the sum in (22) is finite, and that the binomial coefficient in it is zero unless $r_{\infty}$ is small (at most $r_{\alpha}^{g}-r_{g, \alpha}^{\mathrm{Fab}}=2 g-1$ ).

Before proving Theorem 3.11, we digress to observe that just the "shape" of the formula (22) quickly yields the result (I) promised in Section 1 .

Theorem 3.12 (Socle statement for $\left.\mathcal{M}_{g, n}^{r t}\right) \cdot R_{2 g-1}\left(\mathcal{M}_{g, n}^{r t}\right) \cong \mathbb{Q}$.

Proof. We shall show that any monomial in the $\psi$-classes (pushed forward to $\mathcal{M}_{g, 1}$ ) is a multiple of $\mathbb{G}_{g, 1}$. This (with Faber's Non-vanishing Theorem 2.2 and Remark 2.3 (iii)) implies that $R_{2 g-1}\left(\mathcal{M}_{g, n}^{r t}\right)$ is generated by a single element. In particular, $\mathbb{G}_{g, 1} \neq 0$ and

$$
\mathbb{Q} \stackrel{\times \mathbb{G}_{g, 1}}{\longrightarrow} R_{2 g-1}\left(\mathcal{M}_{g, 1}\right)
$$

is an isomorphism.

We show first that $\mathbb{P}_{n}^{g}(\alpha)$ is a multiple of $\mathbb{G}_{g, 1}$ for each partition $\alpha$, by induction on $|\alpha|=\sum_{i} \alpha_{i}$. Now $\mathbb{F}^{g, \alpha}$ is a multiple of $\mathbb{G}_{g, 1}$ (Thm. (3.8), and the contribution of the unique graph with $r_{\infty}=0$ ("the simplest graph in $\mathcal{T}_{g, m}$ ") to Theorem 3.11 is a non-zero multiple of $\mathbb{P}_{n}^{g}(\alpha)$. The contribution of any other graph is a multiple of $\mathbb{P}_{n^{\prime}}^{g}\left(\alpha^{\prime}\right)$ for some smaller $\left|\alpha^{\prime}\right|$. Thus, by induction, $\mathbb{P}_{n}^{g}(\alpha)$ is a multiple of $\mathbb{G}_{g, 1}$ as desired. 
Next, we apply the "polynomiality trick" used in GV1] and GV3. We fix $g$ and $n$, and hence the polynomial $\mathbb{P}_{n}^{g}(\cdot)$. For arbitrary choices of $\alpha_{1}, \ldots, \alpha_{n}, \mathbb{P}_{n}^{g}(\alpha)$ is a multiple of $\mathbb{G}_{g, 1}$. But by knowing enough values of a polynomial of known degree, we can determine its coefficients as linear combinations of these values. Hence all coefficients of $\mathbb{P}_{n}^{g}(\alpha)$ are multiples of $\mathbb{G}_{g, 1}$, and in particular, the monomials in $\psi$-classes (of degree $g-2+n$ ) are multiples of $\mathbb{G}_{g, 1}$.

Proof of Theorem 3.11. We apply relative virtual localization as developed in GV3 (based on the foundational $[\mathrm{GrP}]$ ). As with many virtual localization calculations, Faber classes will be expressed as sums over certain graphs. We show that the set of trees $\mathcal{T}_{g, m}$ is precisely the set of graphs that is required for this purpose by supplying a geometric meaning to the vertices, edges, partitions, weights and constants associated with $\mathfrak{t} \in \mathcal{T}_{g, m}$ through a geometry-combinatorics lexicon.

Classification of torus-fixed loci: We shall have a contribution from each torus-fixed locus of stable relative maps. Recall that Faber-Hurwitz classes are defined by considering the pullback of a linear space under the branch map, applied to the virtual fundamental class of the moduli space of stable relative maps (equ. (8)). As in the proof of the "tautological vanishing theorem" of GV3, we choose a linearization on the branch-class that corresponds to requiring the $r_{g, \alpha}^{\mathrm{Fab}}=r_{\alpha}^{g}-2 g+1$ fixed branch points to go to 0 . Hence in any contributing torus-fixed locus, the amount of branching over $\infty$ is at most $2 g-1$.

We now classify the fixed loci which can appear in the "rational tails" case, where we must have a smooth irreducible component of genus $g$, and then consider the evaluation of their contributions (L1 to L5 below), although some require further elaboration. Fixed loci correspond to maps of the following sort (see Figure 6), and in each case we shall see that the same statements hold for both the left hand side (the simple case) and the right hand side (the composite case) of Figure 6, although the arguments differ slightly. The components mapping surjectively onto $\mathbb{P}^{1}$ are trivial covers (see L1). Over 0, there can be smooth points (as in Figure 6(a)) (see L3), nodes (Figure 6(b)), or contracted components (Figure 6(c)) (see L2). Over $\infty$, either there is no "sprouting", and the preimage of $\infty$ consists of smooth points (the "simple" case, see the left side of Figure 6), or the target "sprouts", and we obtain a relative stable map to an unrigidified target (that we denote by $T_{R}$ ), with possibly-disconnected source (the "composite" case, see the right side of Figure 6) (see L4). In the composite case, let $z$ be the node where the "sprouted" portion $T_{R}$ of the target meets the "unsprouted" portion. If $T_{L}$ denotes the "unsprouted" portion of $T$, then $\infty_{T_{L}}$ and $0_{T_{R}}$ are identified. Note that in the composite case, the sprouted portion of $T$ need not be a single $\mathbb{P}^{1}$; it could be a chain of $\mathbb{P}^{1}$ 's.

Over $\infty$, we can have no genus $g$ components by Remark 3.4 - the only possible branching is over the two endpoints $\left(z\right.$ and $\left.\infty_{T}\right)$, together with at most $2 g-1$ more. Thus the genus $g$ component must lie over 0 , and all irreducible curves over $\infty$ must be genus 0 . (In particular, in the second figure in Figure 6, there cannot be any contracted components mapping to $T_{R}$, so the picture is misleading.)

Geometry-combinatorics lexicon: With the following combinatorial elements, we associate the following geometrical information. 


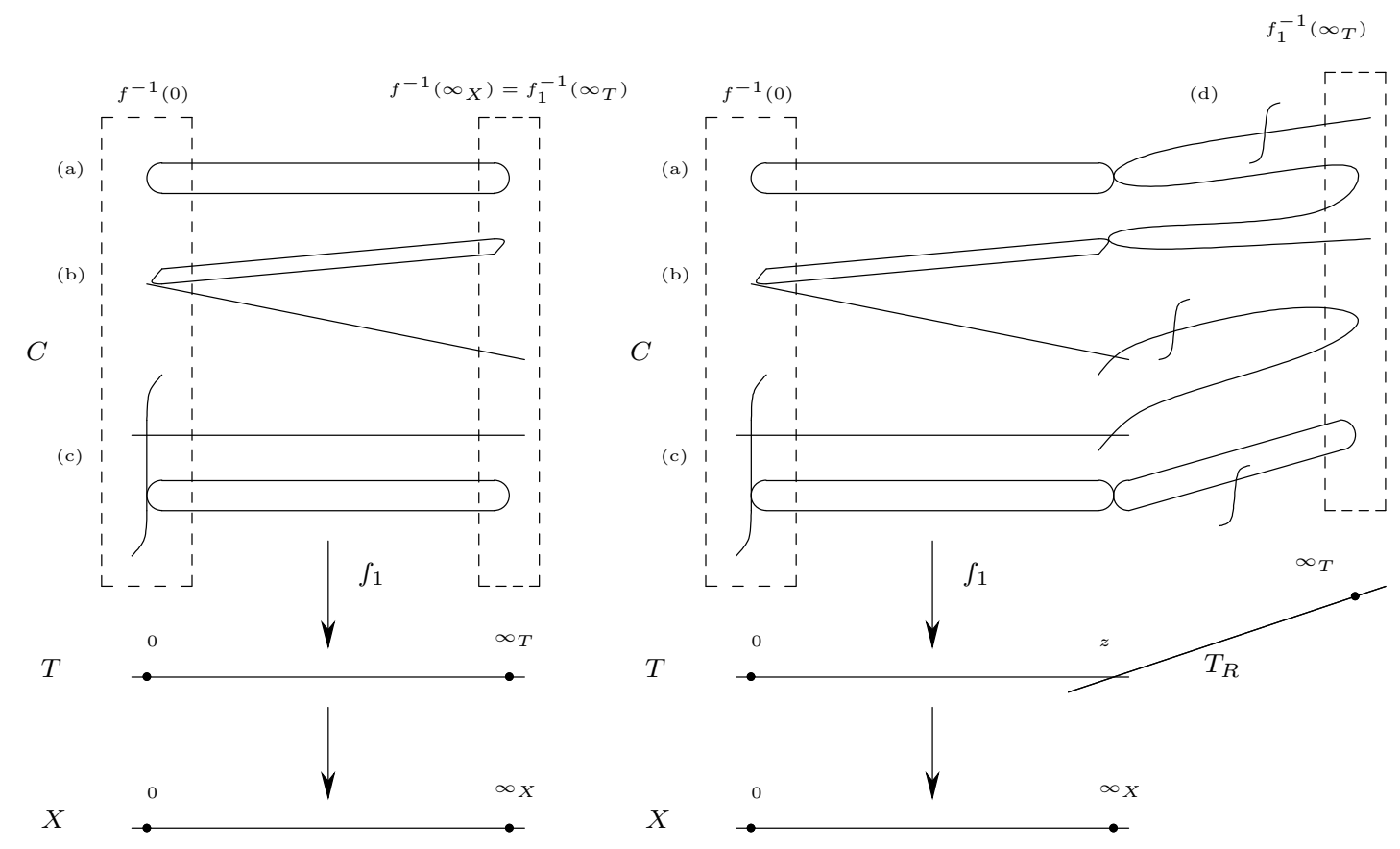

Figure 6. Sketches of two examples of torus-fixed relative stable maps to $X=$ $\left(\mathbb{P}^{1}, \infty\right) . T$ is the "sprouted target".

Vertices and edges of $\mathfrak{t} \in \mathcal{T}_{g, m}$ :

- 0-vertex: $\leftrightarrow$ connected component of the preimage of 0 (contracted curve, node or smooth point).

$-\infty$-vertex: $\leftrightarrow$ connected component of pre-images of $\infty_{X}$.

- t-vertex: $\leftrightarrow$ preimage of $\infty_{T}$.

- $0 \infty$-edge: $\leftrightarrow$ trivial cover of $\mathbb{P}^{1}$; edge joins vertices corresponding to the loci it meets.

- root $\bullet: \leftrightarrow$ genus $g$ (contracted) curve.

Edge weights:

$\overline{- \text { weight } \epsilon(e)}$ on the $0 \infty$-edge $e: \leftrightarrow$ degree of corresponding trivial cover.

- weight on an $\infty$-edge: $\leftrightarrow$ contribution to ramification over $\infty_{X}$ on component specified by the $\infty$-vertex.

Partitions:

$-\delta^{v}$ for a 0 -vertex $v$ : Comb. formed by weights on $0 \infty$-edges incident with $v$. Geom. formed by the degrees of the trivial covers meeting that component.

$-\beta^{v}$ for an $\infty$-vertex $v$ : Comb. formed by weights on $0 \infty$-edges incident with $v$. Geom. formed by the degrees of the trivial covers meeting that component.

$-\gamma^{v}$ for an $\infty$-vertex $v$ : Comb. formed by weights on $\infty t$-edges incident with $v$. Geom. specifies ramification over $\infty_{X}$ on that component.

- $\alpha$ : Comb. see equation (18). Geom. ramification over $\infty$.

Conditions:

$-\left|\beta^{v}\right|=\left|\gamma^{v}\right|$ : Comb. see equation (16). Geom. the degree of the map from this component to $T_{R}$ is $\left|\beta^{v}\right|$ (by examining the preimage of $z$ ) and $\left|\gamma^{v}\right|$ (from the preimage of $\infty_{T}$ ). 
Constants:

$-\eta_{0}(\mathfrak{t}):$ Comb. number of non-root 0-vertices. Geom. number of connected components of the preimage of 0 excluding the component containing the contracted genus $g$ curve.

- n: Comb. $l(\alpha)$. Geom. number of pre-images of $\infty_{T}$.

$-m$ : Comb. degree of the root-vertex $\bullet$ (note $m \leq n)$. Geom. number of trivial covers of $\mathbb{P}^{1}$ meeting the genus $g$ contracted curve.

$-r_{\infty}$ : Comb. see equation (17). Geom. the total branching over $\infty_{X}$.

$-r_{\delta^{v}}^{0}$ for a 0 -vertex $v$ : Comb. see equation (4). Geom. the total branching contributed by the component corresponding to $v$.

$-r_{\gamma^{v}, \beta^{v}}^{0}$ for an $\infty$-vertex $v$ : Comb. see equation (6). Geom. the total branching contributed by the component corresponding to $v$

$-d$ : Comb. $d=|\alpha|$. Geom. degree of the relative stable map

Relative virtual localization: Relative virtual localization ([GV3, Thm. 3.6], see [GV3, Sec. 3.7] for the special case of target $\mathbb{P}^{1}$, and $[\mathrm{GrP}$, Sec. 4] for the non-relative case) tells us that the contribution of a fixed locus can be deduced by looking at the various parts of Figure 6. In what follows, $t$ is the generator of the equivariant cohomology (or Chow) ring of a point, $t \in A_{\mathbb{C}^{*}}^{1}(p t)=\mathbb{Q}[t]$, although we will quickly forget the $t$. The relative virtual localization formula (abbreviated to $\mathbf{L}$ below) gives the following contributions associated with the salient "parts" of the graph. Each of the factors in the summand of (22) will be readily derivable, with the exception of the sign and $D(\mathfrak{t})$ which will require more attention.

Items $\mathbf{L} \mathbf{1}$ to $\mathbf{L} \mathbf{4}$ below come from the description of the fixed loci, and L5 arises from the cohomology class corresponding to fixing some branch points. Moreover, L1 to L3 hold for both the simple and the composite case.

The results for the simple case and the composite case are the same, but the arguments are slightly different.

L1: For each trivial cover of the target $\mathbb{P}^{1}$ of degree $a$, we have contribution $a^{a} /\left(a ! t^{a}\right)$. Hence we obtain the product $\prod \frac{q^{q}}{q !}$ in (22) by collecting those contributions associated with the (genus g) root 0-vertex.

L2: For each contracted curve above 0 of genus $h$ (Figure 6(c)), meeting trivial covers (components mapping surjectively onto $\mathbb{P}^{1}$ ) of degree $\alpha_{1}, \ldots, \alpha_{m}$ respectively, we have a contribution

$$
t^{-1}\left(t^{h}-\lambda_{1} t^{h-1}+\cdots+(-1)^{h} \lambda_{h}\right) \prod_{i=1}^{m} \frac{t}{t / \alpha_{i}-\psi_{i}} .
$$

This contribution is on the factor $\overline{\mathcal{M}}_{h, m}$ corresponding to the contracted curve. We will have $h=0$ or $h=g$, as all components have one of these two genera.

L3: For each node above 0 (Figure 6(b)) joining trivial covers of degrees $\alpha_{1}$ and $\alpha_{2}$, we get a contribution of $t^{-1} \alpha_{1} \alpha_{2} /\left(\alpha_{1}+\alpha_{2}\right)$. For each smooth point above 0 (Figure 6(a)), on a trivial cover of degree $\alpha_{1}$, we get $t^{-1} / \alpha_{1}$. Using the contribution $\prod\left(\alpha_{i}^{\alpha_{i}} / \alpha_{i} !\right)$ from L1, we obtain the factor $H_{\delta^{j}}^{0} / r_{\delta^{j}}^{0}$ ! in (22) for each (non-root) 0 -vertex of degree 1 or 2 , using the formula (11) for genus 0 single Hurwitz numbers. We also obtain the product of the $\epsilon(e)$ in (22) corresponding to $0 \infty$-edges $e$ meeting a degree 1 or 2 (genus 0 , non-root) 0-vertex.

L4: In the composite case (Figure 6(d)), we have a contribution of $1 /\left(-t-\psi_{z}\right.$ ), where $\psi_{z}$ is the first Chern class of the line bundle corresponding to the cotangent space of $T_{R}$ at $z$. 
L5: From the pullback of the linear space by the branch morphism (informally, requiring $r_{g, \alpha}^{\mathrm{Fab}}$ branch points to map to 0$)$, we have a contribution of $\left(\left(2 g-r_{\infty}\right) t\right)\left(\left(2 g+1-r_{\infty}\right) t\right) \cdots\left(\left(r_{\alpha}^{g}-r_{\infty}\right) t\right)$ (there are $r_{g, \alpha}^{\mathrm{Fab}}$ factors), from which we obtain $r_{g, \alpha}^{\mathrm{Fab} !}\left(\begin{array}{c}r_{\alpha}^{g}-r_{\infty} \\ r_{g, \alpha}^{\mathrm{Fab}}\end{array}\right)$ in (22).

So L1-L4 arise from the fixed loci, and L5 arises from the cohomology class corresponding to fixing some branch points. We take the product of these contributions, and read off the constant $\left(t^{0}\right)$ term to obtain the contribution of this fixed locus.

The result is a $(2 g-1)$-dimensional class on $\mathcal{M}_{g, n}^{r t}$. One of the ingredients (from L3) is a (tautological) class on $\overline{\mathcal{M}}_{g, m}$ corresponding to the contracted genus $g$ curve mapping to 0 . Now all tautological classes of dimension less than $2 g-1$ vanish on $\overline{\mathcal{M}}_{g, m}^{r t}$ by Remark 2.3(i). Thus a non-zero contribution is possible only by taking the contribution of a class of dimension precisely $2 g-1$ on $\mathcal{M}_{g, m}^{r t}$, and thus the contributions from every other ingredient must have dimension 0. In light of this observation, we list the contributions from each of the parts of Figure 6, ignoring the equivariant parameter $t$. Also, from L3, the contribution by the contracted genus $g$ curve is $\mathbb{P}_{m}^{g}\left(\delta^{0}\right)$ so, with the contribution from $\mathbf{L} 1$, we obtain the term $A(\mathfrak{t})$, a term in (22). In addition, we obtain the product of the $\epsilon(e)$ over those edges $e$ meeting the (genus $g$ ) root 0 -vertex.

From (24) (using $h=0$ ), any contracted genus 0 component over $0 \in \mathbb{P}^{1}$ meeting trivial covers of degrees $\delta_{1}^{j}, \ldots, \delta_{p}^{j}(p \geq 3$ by the stability condition) gives the dimension 0 contribution

$$
\left(\prod_{i=1}^{p} \delta_{i}^{j}\right) \int_{\overline{\mathcal{M}}_{0, p}} \frac{1}{\left(1-\delta_{1}^{j} \psi_{1}\right) \cdots\left(1-\delta_{p}^{j} \psi_{p}\right)}
$$

Combining this with $\prod_{i=1}^{p} \frac{\delta_{i}^{\delta_{i}^{j}}}{\delta_{i}^{j} !}$ from L1, using the ELSV formula [ELSV1, ELSV2, GV2] we obtain $\left(\prod \delta_{i}^{j}\right) H_{\delta^{j}}^{0} / r_{\delta^{j}}^{0}$ !. (We could have bypassed the ELSV formula, using instead the formula (11) for genus 0 Hurwitz numbers and the string equation, given in Proposition 2.6.) Thus we obtain the factor $H_{\delta^{j}}^{0} / r_{\delta^{j}}^{0}$ ! for each non-root 0 -vertex of degree at least three. We also obtain the product of the $\epsilon(e)$ corresponding to $0 \infty$-edges $e$ meeting all (genus 0 , non-root) 0 -vertices of degree at least 3. L3 gives the same values for degree 1 and 2 so, combining the three sources for the $\epsilon$ 's, we now obtain the entire product $B(\mathfrak{t})$, and combining the two sources for the $H_{\delta^{j}}^{0} / r_{\delta^{j}}^{0}$ !, we obtain the entire product $C(\mathfrak{t})$.

The contributions from $\mathbf{L} \mathbf{1}-\mathbf{L} \mathbf{3}$, and $\mathbf{L} \mathbf{5}$ are now exhausted.

It remains to obtain the sign $(-1)^{r_{\infty}}$, the term $D(\mathfrak{t})$, and the division by $\eta_{0}(\mathfrak{t})$ !. To do so, we now appeal to L4. In the composite case (if the target "sprouts", Figure 6 (d)), suppose $\overline{\mathcal{M}}:=\overline{\mathcal{M}}_{\beta, \alpha}$ is the moduli space of relative stable maps to the unrigidified $T_{R}$, where $\beta$ is the kissing partition above $z$. It is the moduli space of relative stable maps, where the source is a disjoint union of genus 0 curves. As always for maps to unrigidified targets, the virtual dimension of this space is one less than the number of "moving branch points" $r_{\overline{\mathcal{M}}}$. Then the contribution is the dimension 0 portion of $\frac{1}{-1-\psi_{z}}[\overline{\mathcal{M}}]^{\text {vir }}$ which is

$$
(-1)^{r} \overline{\mathcal{M}} \psi_{z}^{r \overline{\mathcal{M}}-1}[\overline{\mathcal{M}}]^{\text {vir }}
$$

The sign gives us the factor $\prod(-1)^{r^{r^{i}, \beta^{i}}}$ which, from (17) , is equal to $(-1)^{r_{\infty}}$, the sign in (22) .

The proof of [GV3, Lem. 4.8] (see also [GV3, Fig. 2]) shows that $\psi^{a}$ applied to $\overline{\mathcal{M}}$ can be interpreted as requiring that the target break into $a+1$ components. More precisely, $\psi^{a}$ applied to $[\overline{\mathcal{M}}]^{\text {vir }}$ is the same as gluing virtual fundamental classes of relative stable maps to the $a+1$ 
components of $T_{R}$ (in the same sense as the degeneration formula, with kissing multiplicities arising for each node of the target $\left.T_{R}\right)$, divided by $(a+1)$ !. This latter term gives a factor of $1 / r_{\infty}$ !.

In particular, $\psi^{r_{\overline{\mathcal{M}}}}-1$ corresponds to the target breaking into $r_{\overline{\mathcal{M}}}$ components. Because the resulting map must be stable, there must be some branching on each of these components (away from the nodes of $T_{R}$, and $z$ and $\infty_{T}$ ). Thus as the total amount of branching is $r_{\overline{\mathcal{M}}}$ away from the nodes of $T_{R}$, and there is precisely this number of components, we must have branching number 1 on each irreducible component of $T_{R}$. By Remark 3.3(b), above each component of the $T_{R}$, we must have precisely one almost-trivial cover, along with some trivial covers.

Thus the contribution of (25) is the size of a discrete set (counted modulo automorphisms). This set counts the number of branched covers of $T_{R}$ (a chain of $r_{\overline{\mathcal{M}}} \mathbb{P}^{1}$ 's), with one simple branching on each component, and given branching $\epsilon$ over $z$ (a point at the end of the chain) and $\alpha$ over $\infty_{T}$ (a point at the other end of the chain), satisfying the kissing condition over each node of $T_{R}$. By the gluing formula (or indeed, the much older technique of just studying the degeneration), this is the number of branched covers of $\mathbb{P}^{1}$ by a union of genus 0 curves with branching given by $\epsilon$ and $\alpha$ over two points $z$ and $\infty_{T}$, and simple branching over $r_{\overline{\mathcal{M}}}$ other given fixed points.

We shall now see that this is (up to a combinatorial factor) a product of genus 0 double Hurwitz numbers. Recall that a genus 0 double Hurwitz number

$$
H_{\alpha, \varepsilon}^{0}
$$

(where $\alpha$ and $\varepsilon$ are partitions of some number $e$ ) counts the number of degree $e$ covers of $\mathbb{P}^{1}$ by $\mathbb{P}^{1}$, with branching $\alpha$ at one fixed point, $\varepsilon$ at another, and simple branching at $r_{\alpha, \varepsilon}^{0}$ other fixed points. Suppose we are considering covers by $N \mathbb{P}^{1}$ 's, where component $i$ corresponds to the subpartition $\beta^{i}$ of $\varepsilon$ (over $z$ ) and the subpartition $\gamma^{i}$ of $\alpha$ (over $\infty_{T}$ ). (Thus $\left|\beta^{i}\right|=\left|\gamma^{i}\right|$ is the degree of that subcover, $\varepsilon=\coprod_{i} \beta^{i}$, and $\alpha=\coprod_{i} \gamma^{i}$.) Then component $i$ has simple branching over $r_{\infty}^{i}:=r_{\beta^{i}, \gamma^{i}}^{0}$

of the fixed simple branch points. There are $\left(\begin{array}{c}\sum_{i} r_{\infty}^{i} \\ r_{\infty}^{1}, \ldots, r_{\infty}^{N}\end{array}\right)$ ways of partitioning the branch points into these $N$ sets. Once this partition is chosen, there are $\prod_{i=1}^{N} H_{\beta^{i}, \gamma^{i}}^{0}$ such branched covers. (One caution: we have cavalierly described $H_{\beta^{i}, \gamma^{i}}^{0}$ as enumerating a set. In reality, each cover is counted with multiplicity equal to the inverse of the size of its automorphism group, so $H_{\beta^{i}, \gamma^{i}}^{0}$ need not be integral, and in fact is not precisely for $\beta^{i}=\gamma^{i}=\left(d_{i}\right)$; trivial covers of degree $d_{i}$ "count for" $1 / d_{i}$.) We have obtained the factors $H_{\beta^{k}, \gamma^{k}}^{0}$ and $\left(\begin{array}{c}r_{\infty} \\ r_{\infty}^{1}, \ldots, r_{\infty}^{N}\end{array}\right)$ in (22). (The numerator $r_{\infty}$ ! in the multinomial coefficient cancels the $1 / r_{\infty}$ ! from earlier.) This is the term $D(\mathfrak{t})$.

Finally, the $1 / \eta_{0}(\mathfrak{t}) !$ in $(22)$ is present because the trees have labelled non-root 0 -vertices (Def. 3.10).

\section{Part 3. ALGEBRAIC COMBINATORICS}

At this point, we have defined the Faber-Hurwitz classes $\mathbb{F}^{g, \alpha}$, which "virtually" correspond to "rational tail" curves admitting a branched cover of $\mathbb{P}^{1}$ with branching at $\infty$ corresponding to $\alpha$, and "all but $2 g-1$ branching fixed". Such classes are a multiple of a basic class $\mathbb{G}_{g, 1}$; this multiple is the Faber-Hurwitz number $F^{g, \alpha}$. By degeneration, we have obtained Corollary 3.9, the Degeneration Theorem for the generating series $F^{g}$ for these numbers, involving the genus 0 Hurwitz series $\widehat{H}^{0}$. By localization, we have also obtained Theorem 3.11, the Localization Tree Theorem, which describes these classes (or numbers) as a sum over certain rooted, labelled trees, involving genus 0 Hurwitz, double Hurwitz numbers and the desired intersection numbers (of $\psi$ classes). Theorem 3.12 shows us that we can "invert" this expression, to determine intersection numbers in terms of Hurwitz numbers and double Hurwitz numbers. Our goal is to formalize this. 
The strategy is to show that Localization Tree Theorem and the Degeneration Theorem, taken together, give a non-singular system of linear equations for the top Faber intersection numbers, so it has a unique solution, and that the conjectural values satisfy it.

We accomplish this by a sequence of transformations, which yield a number of refined versions of the Localization Tree Theorem and the Degeneration Theorem. These versions of the Localization Tree Theorem (we say that these are results for the "localization side") are given by the sequence

$$
\text { Thm. 3.11 } \leadsto \text { Thm. 4.4 } \leadsto \text { Cor. 5.1 } \leadsto \text { Cor. } 7.1
$$

These versions of the Degeneration Theorem (we say that these are results for the "degeneration side") are given by the sequence

$$
\text { Thm. 3.7 } \sim \text { Cor. } 3.9 \sim \text { Cor. } 5.2 \sim \text { Lem. } 7.5
$$

\section{EXPONENTIAL GENERATING SERIES FOR LOCALIZATION TREES}

The purpose of this section is to "evaluate" the sum over localization trees that arises from the localization arguments in Theorem 3.11. Localization trees are a class of rooted, labelled trees, and we use the standard multivariate exponential generating series for combinatorial structures with many sets of labels, as well as variants of the standard branch decomposition for rooted trees.

4.1. Exponential generating series and the $\star$-product. For a localization tree $\mathfrak{t}$, let $\eta_{k}(\mathfrak{t})$ denote the number of $t$-vertices in $\mathfrak{t}$ that are incident with an edge of weight $k, k \geq 1$.

Definition 4.1. Let $\mathcal{A}$ be a set of localization trees, with weight function wt. Then the exponential generating series for $\mathcal{A}$ with respect to wt is

$$
[\mathcal{A}, \mathrm{wt}]_{\eta}:=\sum_{\mathfrak{t} \in \mathcal{A}} \frac{p_{1}^{\eta_{1}(\mathfrak{t})} p_{2}^{\eta_{2}(\mathfrak{t})} \cdots}{\eta_{0}(\mathfrak{t}) ! \eta_{1}(\mathfrak{t}) ! \eta_{2}(\mathfrak{t}) ! \cdots} \mathrm{wt}(\mathfrak{t}) .
$$

For now, we shall allow the range of the weight function to be any ring, or even a vector space, and in particular we allow geometric classes. Note that, as a formal power series in $p_{1}, p_{2}, \ldots,[\mathcal{A} \text {, wt }]_{\eta}$ is always well-formed because of the balance condition (16), which ensures that there is only a finite number of localization trees $\mathfrak{t}$ with $\eta_{k}(\mathfrak{t})=i_{k}, k \geq 1$, for each $i_{1}, i_{2}, \ldots$ (so the coefficients are finite sums of weight function values).

Since localization trees are labelled objects, we consider a particular version of the standard $\star$-product for them, which is the Cartesian product together with a "label-distribution" operation. We define this $\star$-product as follows. Consider two localization trees $\mathfrak{t}_{1}$ and $\mathfrak{t}_{2}$. Suppose that $\eta_{k}\left(\mathfrak{t}_{1}\right)=i_{k}$, and $\eta_{k}\left(\mathfrak{t}_{2}\right)=j_{k}$, for $k \geq 0$. Now choose subsets $\alpha_{k} \subseteq\left[i_{k}+j_{k}\right]$, with $\left|\alpha_{k}\right|=i_{k}$, for $k \geq 0$, and let $\beta_{k}=\left[i_{k}+j_{k}\right] \backslash \alpha_{k}$ (so $\left|\beta_{k}\right|=j+k$ ) (we use the notation $[n]=\{1, \ldots, n\}$ ). Let $\alpha\left(\mathfrak{t}_{1}\right)$ be the tree obtained from $\mathfrak{t}_{1}$ by relabelling the labelled vertices as follows: replace the label $m$ on a non-root 0 -vertex by the $m$ th smallest element of $\alpha_{0}$, for $m=1, \ldots, i_{0}$; replace the label $m$ on a $t$-vertex incident with an edge of weight $k$ by the $m$ th smallest element of $\alpha_{k}$, for $m=1, \ldots, i_{k}$, $k \geq 1$. Let $\beta\left(\mathfrak{t}_{2}\right)$ be the tree obtained from $\mathfrak{t}_{2}$ by relabelling the labelled vertices with the elements of $\beta_{0}, \beta_{1}, \ldots$, in the analogous manner. We call $(\alpha, \beta)$ a compatible relabelling (of $\left(\mathfrak{t}_{1}, \mathfrak{t}_{2}\right)$ ). Where convenient, we also refer to $\mathfrak{t}_{1}$ and $\mathfrak{t}_{2}$ as canonically labelled.

Definition 4.2. Let $\mathcal{A}, \mathcal{B}$ be sets of localization trees. Then

$$
\mathcal{A} \star \mathcal{B}:=\left\{\left(\alpha\left(\mathfrak{t}_{1}\right), \beta\left(\mathfrak{t}_{2}\right)\right):\left(\mathfrak{t}_{1}, \mathfrak{t}_{2}\right) \in \mathcal{A} \times \mathcal{B},(\alpha, \beta) \text { a compatible relabelling of }\left(\mathfrak{t}_{1}, \mathfrak{t}_{2}\right)\right\} .
$$

The reason for using exponential generating series for labelled combinatorial objects is the Product Lemma, given in the following result. For a proof, see, e.g., Goulden and Jackson GJ1, Lem. 3.2.11]. 
Lemma 4.3 (Product Lemma (for localization tree generating series)). Let $\mathcal{S}, \mathcal{A}, \mathcal{B}$ be sets of localization trees, with weight functions $\mathrm{wt}, \mathrm{wt}_{1}, \mathrm{wt}_{2}$, respectively. Suppose there is a bijection

$$
\mathcal{S} \stackrel{\sim}{\longrightarrow} \mathcal{A} \star \mathcal{B}: \mathfrak{t} \mapsto\left(\alpha\left(\mathfrak{t}_{1}\right), \beta\left(\mathfrak{t}_{2}\right)\right),
$$

subject to $\mathrm{wt}(\mathfrak{t})=\mathrm{wt}_{1}\left(\mathfrak{t}_{1}\right) \mathrm{wt}_{2}\left(\mathfrak{t}_{2}\right)$ and $\eta_{k}(\mathfrak{t})=\eta_{k}\left(\mathfrak{t}_{1}\right)+\eta_{k}\left(\mathfrak{t}_{2}\right), k \geq 0$. Then

$$
[\mathcal{S}, \mathrm{wt}]_{\eta}=\left[\mathcal{A}, \mathrm{wt}_{1}\right]_{\eta}\left[\mathcal{B}, \mathrm{wt}_{2}\right]_{\eta} .
$$

For any set $\mathcal{S}$ of localization trees $\mathcal{S}$, let $\mathcal{S}^{\star k}:=\mathcal{S} \star \cdots \star \mathcal{S}$, where there are $k \mathcal{S}$ 's in this $k$-fold $\star$-product. We define $\mathcal{U}_{k}(\mathcal{S}):=\mathcal{S}^{\star k} / \mathfrak{S}_{k}$, under the natural action of $\mathfrak{S}_{k}$, for $k \geq 0$.

4.2. Branch decompositions for localization trees. In order to decompose localization trees, we require three variants of the standard branch decomposition, described below. These are formalized as combinatorial mappings, called $\Omega^{\mathrm{br}}, \Omega_{0}^{\mathrm{br}}$ and $\Omega_{\infty}^{\mathrm{br}}$.

First, we give $\Omega^{\text {br }}$ : If the root-vertex $\bullet$ and incident edges are deleted from a localization tree $\mathfrak{t}$, then we obtain a list $\left(\mathfrak{t}_{1}, \ldots, \mathfrak{t}_{m}\right)$ of rooted trees on mutually distinct sets of vertices, each inheriting as root vertex its unique vertex that was adjacent to $\bullet$ in $\mathfrak{t}$, where $m$ is the degree of the root-vertex of $\mathfrak{t}$. Now let $\mathfrak{t}_{i}^{\prime}$ be obtained from $\mathfrak{t}_{i}$ by joining the root vertex of $\mathfrak{t}_{i}$ to a new copy of $\bullet$ (which becomes the new root-vertex of $\mathfrak{t}_{i}^{\prime}$ ), joined by an edge whose weight is equal to the weight of the edge joining $\bullet$ to the root-vertex of $\mathfrak{t}_{i}$ in $\mathfrak{t}$. Since $\bullet$ is unlabelled in $\mathfrak{t}$, then $\left(\mathfrak{t}_{1}^{\prime}, \ldots, \mathfrak{t}_{m}^{\prime}\right)$ is equal to $\left(\alpha_{1}\left(\mathfrak{t}_{1}^{\prime \prime}\right), \ldots, \alpha_{m}\left(\mathfrak{t}_{m}^{\prime \prime}\right)\right)$, a compatible relabelling of canonical localization trees $\left(\mathfrak{t}_{1}^{\prime \prime}, \ldots, \mathfrak{t}_{m}^{\prime \prime}\right)$. Then we define $\Omega^{\mathrm{br}}(\mathfrak{t})=\left(\alpha_{1}\left(\mathfrak{t}_{1}^{\prime \prime}\right), \ldots, \alpha_{m}\left(\mathfrak{t}_{m}^{\prime \prime}\right)\right)$. This corresponds to removing the root from the tree, and describing the remainder as a list of trees.

Second, we give $\Omega_{0}^{\mathrm{br}}$ : Suppose that $\mathrm{t}$ is a localization tree whose root-vertex $\bullet$ is a monovalent 0 -vertex. Let the $\infty$-vertex adjacent to $\bullet$ be $u$, and let $\mathfrak{t}^{\prime}$ be the tree obtained by deleting $\bullet$ and incident edge from $\mathfrak{t}$, and deleting all $t$-vertices adjacent to $u$, together with their incident edges, and rooting the resulting tree at $u$. Now form a new graph $G$, containing these deleted $t$-vertices (labelled as in $\mathfrak{t}$ ), joined to a new $\infty$-vertex $w$ by an edge of the same weight as the deleted incident edge in $\mathfrak{t}$. Then we define $\Omega_{0}^{\mathrm{br}}(\mathfrak{t})=\left(G, \Omega^{\mathrm{br}}\left(\mathfrak{t}^{\prime}\right)\right)$. Now consider partition $\gamma=\left(1^{a_{1}} 2^{a_{2}} \ldots\right)$, and let $\mathfrak{w}_{\gamma}$ be the graph consisting of a single $\infty$-vertex, joined by an edge of weight $k$ to $a_{k}$ canonically labelled monovalent $t$-vertices, $k \geq 1$. Then note that $\Omega_{0}^{\mathrm{br}}(\mathfrak{t})=\left(\alpha_{0}\left(\mathfrak{w}_{\gamma}\right), \alpha_{1}\left(\mathfrak{t}_{1}^{\prime \prime}\right), \ldots, \alpha_{m}\left(\mathfrak{t}_{m}^{\prime \prime}\right)\right)$, a compatible relabelling of the canonical $\left(\mathfrak{w}_{\gamma}, \mathfrak{t}_{1}^{\prime \prime}, \ldots, \mathfrak{t}_{m}^{\prime \prime}\right)$, where $\gamma=\gamma^{u}(\mathfrak{t}),|\gamma|=\left|\beta^{u}(\mathfrak{t})\right|$ (because of the balance condition (16) at $u$ ), and $\beta^{u}(\mathfrak{t})$ has $m+1$ parts, $m \geq 0$.

Third, we give $\Omega_{\infty}^{\mathrm{br}}$ : Suppose that $\mathfrak{t}$ is a localization tree whose root-vertex $\bullet$ is a monovalent $\infty$ vertex. Let the 0 -vertex adjacent to $\bullet$ be $v$, with label $i$, then let $\mathfrak{t}^{\prime}$ be the tree obtained by removing - from $\mathfrak{t}$, and removing the label from the vertex $v$, and rooting the resulting tree at $v$. Let $\mathfrak{v}_{i}$ be the graph consisting of a single, 0-vertex, labelled $i$. Then we define $\Omega_{\infty}^{\mathrm{br}}(\mathfrak{t})=\left(\mathfrak{v}_{i}, \Omega^{\mathrm{br}}\left(\mathfrak{t}^{\prime}\right)\right)$, and note that $\Omega_{\infty}^{\mathrm{br}}(\mathfrak{t})=\left(\alpha_{0}\left(\mathfrak{v}_{1}\right), \alpha_{1}\left(\mathfrak{t}_{1}^{\prime \prime}\right), \ldots, \alpha_{m}\left(\mathfrak{t}_{m}^{\prime \prime}\right)\right)$, a compatible relabelling of the canonical $\left(\mathfrak{v}_{1}, \mathfrak{t}_{1}^{\prime \prime}, \ldots, \mathfrak{t}_{m}^{\prime \prime}\right)$, where $\delta^{v}(\mathfrak{t})$ has $m+1$ parts, $m \geq 0$.

4.3. Generating series form for the Localization Tree Theorem. In the next result, Theorem 4.4, which is the second form of the Localization Tree Theorem, we introduce a generating series, $\mathbb{T}^{g}$, for the tree summation that arose in Theorem 3.11, which was the first form of the Localization Tree Theorem. We also introduce two ancillary classes of generating series $f_{j}, g_{j}, j \geq 1$. These are all exponential generating series with respect to particular weight functions, and the proofs of the equations that relate them are combinatorial, applying the variants of the branch decomposition, together with the $\star$-product and the Product Lemma. However, the statement of Theorem 4.4 is purely algebraic (without reference to trees), and the series $\mathbb{T}^{g}, f_{j}, g_{j}$ are all uniquely defined by the equations in the statement. Note that the coefficients in $\mathbb{T}^{g}$ are geometric classes, while the coefficients in $f_{j}, g_{j}, F^{g}$ are rationals. 
The statement of the theorem involves the generating series $\widehat{H}^{0}$ for genus 0 Hurwitz numbers, defined in (14), and the generating series for genus 0 double Hurwitz numbers, given by

$$
H^{0}(z, u ; \mathbf{p} ; \mathbf{q}):=\sum_{\substack{\alpha, \beta \in \mathcal{P} \\|\alpha|=|\beta|}} z^{|\beta|} p_{\alpha} q_{\beta} u^{l(\beta)} \frac{H_{\alpha, \beta}^{0}}{r_{\alpha, \beta}^{0} !|\operatorname{Aut} \alpha||\operatorname{Aut} \beta|},
$$

where $\mathbf{p}=\left(p_{1}, p_{2}, \ldots\right), \mathbf{q}=\left(q_{1}, q_{2}, \ldots\right)$, and $r_{\alpha, \beta}^{0}$ is defined in (6) .

Theorem 4.4 (Localization Tree Theorem - functional equations). For $g \geq 1$, we have

$$
\text { a) } \quad F^{g}(z ; \mathbf{p}) \mathbb{G}_{g, 1}=\left[u^{2 g-1}\right] \mathbb{T}^{g}\left(\frac{z}{1-u},-u ; \frac{-u}{1-u} \mathbf{p}\right) \text {, }
$$

where

$$
\text { b) } \mathbb{T}^{g}(z, u ; \mathbf{p})=\sum_{n \geq 1} \frac{1}{n !} \sum_{\substack{a_{1}, \ldots, a_{n}, k \geq 0, a_{1}+\cdots+a_{n}+k=g-2+n}}(-1)^{k}\left\langle\tau_{a_{1}} \cdots \tau_{a_{n}} \lambda_{k}\right)_{g}^{\mathbb{F a b}} \prod_{j=1}^{n} \xi^{\left(a_{j}\right)}(z, u ; \mathbf{p})
$$

with

$$
\xi^{(i)}(z, u ; \mathbf{p}):=\sum_{j \geq 1} \frac{j^{j+i}}{j !} f_{j}(z, u ; \mathbf{p}), \quad i \geq 0,
$$

and, for $j \geq 1, c) \quad f_{j}=\left.u^{-2}\left(j \frac{\partial}{\partial q_{j}} H^{0}(z, u ; \mathbf{p} ; \mathbf{q})\right)\right|_{q_{i}=g_{i}, i \geq 1}, \quad$ d) $g_{j}=\left.\left(j \frac{\partial}{\partial q_{j}} \widehat{H}^{0}(1 ; \mathbf{q})\right)\right|_{q_{i}=f_{i}, i \geq 1}$, with $f_{j}=f_{j}(z, u ; \mathbf{p}), g_{j}=g_{j}(z, u ; \mathbf{p})$.

Proof. As special cases of weight functions for localization trees $\mathfrak{t}$, consider $\mathrm{wt}_{g}(\mathfrak{t}):=A(\mathfrak{t}) \mathrm{wt}_{0}(\mathfrak{t})$, $\mathrm{wt}_{0}(\mathfrak{t}):=B(\mathfrak{t}) C^{\ddagger}(\mathfrak{t}) D(\mathfrak{t}) \prod_{v \in \mathcal{V}_{\infty}} u^{l\left(\beta^{v}\right)-2} z^{\left|\beta^{v}\right|}$, and $\mathrm{wt}_{\infty}(\mathfrak{t}):=B(\mathfrak{t}) C(\mathfrak{t}) D^{\ddagger}(\mathfrak{t}) \prod_{v \in \mathcal{V}_{\infty}}^{\ddagger} u^{l\left(\beta^{v}\right)-2} z^{\left|\beta^{v}\right|}$, where $A, B, C, D$ were defined in (21), (20). Now define generating series

$$
\mathbb{T}^{g}(z, u ; \mathbf{p}):=\left[\cup_{m \geq 1} \mathcal{T}_{g, m}, \mathrm{wt}_{g}\right]_{\eta}, \quad g \geq 1, \quad f_{j}:=\left[\mathcal{T}_{0, j}, \mathrm{wt}_{0}\right]_{\eta}, \quad g_{j}:=\left[\mathcal{T}_{\infty, j}, \mathrm{wt}_{\infty}\right]_{\eta}, \quad j \geq 1 .
$$

For part (a), in $\mathbb{T}^{g}$ we have $p_{1}^{\eta_{1}(\mathfrak{t})} p_{2}^{\eta_{2}(\mathfrak{t})} \cdots / \eta_{1}(\mathfrak{t}) ! \eta_{2}(\mathfrak{t}) ! \cdots=p_{\alpha} / \mid$ Aut $\alpha \mid$, from (18). Thus, for $g \geq 1$ and $|\alpha|=d \geq 1$, we have $\mathbb{F}^{g, \alpha}=r_{g, \alpha}^{\mathrm{Fab}} ! \mid$ Aut $\alpha \mid \sum_{r_{\infty}}\left(\begin{array}{c}r_{\alpha}^{g}-r_{\infty} \\ r_{g, \alpha}^{\mathrm{Fab}}\end{array}\right)(-1)^{r_{\infty}}\left[p_{\alpha} u^{r_{\infty}-l(\alpha)} z^{d}\right] \mathbb{T}^{g}(z, u ; \mathbf{p})$, from Theorem 3.11. But $\left(\begin{array}{c}r_{\alpha}^{g}-r_{\infty} \\ r_{g, \alpha}^{\mathrm{Fab}}\end{array}\right)=\left[u^{2 g-1-r_{\infty}}\right](1-u)^{-(d+l(\alpha))}$, so $\mathbb{F}^{g, \alpha}=r_{g, \alpha}^{\mathrm{Fab}} ! \mid$ Aut $\alpha \mid\left[p_{\alpha} u^{2 g-1} z^{d}\right]$ $\mathbb{T}^{g}\left(\frac{z}{1-u},-u ; \frac{-u}{1-u} \mathbf{p}\right)$. Then part (a) follows from (15) and (13).

For part (b), define $\mathcal{T}_{g, \delta}$ to be the set of localization trees $\mathfrak{t}$ in $\mathcal{T}_{g, m}$ with $\delta^{\bullet}(\mathfrak{t})=\delta$, for any partition $\delta$ with $m$ parts, $m \geq 1$. Then variant $\Omega^{\text {br }}$ of the branch decomposition gives a bijection

$$
\Omega^{\mathrm{br}}: \mathcal{T}_{g, \delta} \stackrel{\sim}{\longrightarrow} \mathcal{U}_{a_{1}}\left(\mathcal{T}_{0,1}\right) \star \mathcal{U}_{a_{2}}\left(\mathcal{T}_{0,2}\right) \star \cdots: \mathfrak{t} \mapsto\left(\alpha_{1}\left(\mathfrak{t}_{1}^{\prime \prime}\right), \ldots, \alpha_{m}\left(\mathfrak{t}_{m}^{\prime \prime}\right)\right),
$$

where $\delta=\left(1^{a_{1}} 2^{a_{2}} \ldots\right)$ with $a_{1}+a_{2}+\cdots=m$. It is straightforward to check in this bijection that $\eta_{k}(\mathfrak{t})=\eta_{k}\left(\mathfrak{t}_{1}^{\prime \prime}\right)+\cdots+\eta_{k}\left(\mathfrak{t}_{m}^{\prime \prime}\right)$, for $k \geq 0$. Moreover, $\operatorname{wt}_{g}(\mathfrak{t})=\mathbb{P}_{m}^{g}\left(1^{a_{1}} 2^{a_{2}} \cdots\right)\left(\prod_{j \geq 1}\left(j^{j} / j !\right)^{a_{j}}\right)$ $\mathrm{wt}_{0}\left(\mathfrak{t}_{1}^{\prime \prime}\right) \cdots \mathrm{wt}_{0}\left(\mathfrak{t}_{m}^{\prime \prime}\right)$, so from the Product Lemma we obtain

$$
\left[\mathcal{T}_{g, \delta}, \mathrm{wt}_{g}\right]_{\eta}=\mathbb{P}_{m}^{g}\left(1^{a_{1}} 2^{a_{2}} \ldots\right) \prod_{j \geq 1} \frac{\left(j^{j} f_{j} / j !\right)^{a_{j}}}{a_{j} !}
$$

and part (b) follows by summing this over all $a_{1}, a_{2}, \ldots \geq 0$ with $a_{1}+a_{2}+\cdots=m$, and $m \geq 1$, and applying (19).

For part (c), for $j \geq 1$, variant $\Omega_{0}^{\mathrm{br}}$ of the branch decomposition gives a bijection

$$
\Omega_{0}^{\mathrm{br}}: \mathcal{T}_{0, j} \stackrel{\sim}{\longrightarrow} \bigcup\left\{\mathfrak{w}_{\gamma}\right\} \star \mathcal{U}_{b_{1}}\left(\mathcal{T}_{\infty, 1}\right) \star \mathcal{U}_{b_{2}}\left(\mathcal{T}_{\infty, 2}\right) \star \cdots: \mathfrak{t} \mapsto\left(\alpha_{0}\left(\mathfrak{w}_{\gamma}\right), \alpha_{1}\left(\mathfrak{t}_{1}^{\prime \prime}\right), \ldots, \alpha_{m}\left(\mathfrak{t}_{m}^{\prime \prime}\right)\right),
$$


where the union is over all $\gamma=\left(1^{a_{1}} 2^{a_{2}} \cdots\right), \beta=\left(1^{b_{1}} \cdots(j-1)^{b_{j-1}} j^{b_{j}+1}(j+1)^{b_{j+1}} \cdots\right)$ with $|\gamma|=$ $|\beta| \geq j$, and $l(\beta)=m+1, m \geq 0$ (here, $\gamma=\gamma^{u}(\mathfrak{t})$ and $\beta=\beta^{u}(\mathfrak{t})$, where $u$ is the $\infty$-vertex adjacent to $\bullet$ in $\mathfrak{t}$, as given in the description of $\Omega_{0}^{\mathrm{br}}$ above). It is straightforward to check in this bijection that $\eta_{k}(\mathfrak{t})=\eta_{k}\left(\mathfrak{w}_{\gamma}\right)+\eta_{k}\left(\mathfrak{t}_{1}^{\prime \prime}\right)+\cdots+\eta_{k}\left(\mathfrak{t}_{m}^{\prime \prime}\right)$, for $k \geq 0$. Moreover, $\mathrm{wt}_{0}(\mathfrak{t})=j\left(H_{\gamma, \beta}^{0} u^{l(\beta)-2} z^{|\beta|} / r_{\gamma, \beta}^{0} !\right)$ $\mathrm{wt}_{\infty}\left(\mathfrak{t}_{1}^{\prime \prime}\right) \cdots \mathrm{wt}_{\infty}\left(\mathfrak{t}_{m}^{\prime \prime}\right)$, so from the Product Lemma we obtain

$$
f_{j}=u^{-2} j \sum \frac{H_{\gamma, \beta}^{0} u^{l(\beta)} z^{|\beta|}}{r_{\gamma, \beta}^{0} !} \prod_{i \geq 1} \frac{p_{i}^{a_{i}}}{a_{i} !} \frac{g_{i}^{b_{i}}}{b_{i} !},
$$

since $\left[\mathfrak{w}_{\gamma}, 1\right]_{\eta}=\prod_{i \geq 1} \frac{p_{i}^{a_{i}}}{a_{i} !}$, and part (c) follows immediately from (27).

For part $(\mathrm{d})$, variant $\Omega_{\infty}^{\mathrm{br}}$ of the branch decomposition gives a bijection

$$
\Omega_{\infty}^{\mathrm{br}}: \mathcal{T}_{\infty, j} \stackrel{\sim}{\longrightarrow} \bigcup\left\{\mathfrak{v}_{1}\right\} \star \mathcal{U}_{a_{1}}\left(\mathcal{T}_{0,1}\right) \star \mathcal{U}_{a_{2}}\left(\mathcal{T}_{0,2}\right) \star \cdots: \mathfrak{t} \mapsto\left(\alpha_{0}\left(\mathfrak{v}_{1}\right), \alpha_{1}\left(\mathfrak{t}_{1}^{\prime \prime}\right), \ldots, \alpha_{m}\left(\mathfrak{t}_{m}^{\prime \prime}\right)\right)
$$

where the union is over all $\delta=\left(1^{a_{1}} \cdots(j-1)^{a_{j-1}} j^{a_{j}+1}(j+1)^{a_{j+1}} \ldots\right)$ with $|\delta| \geq j$, and $l(\delta)=m+1$, $m \geq 0$. (Here, $\delta=\delta^{v}(\mathfrak{t})$, where $v$ is the 0 -vertex adjacent to $\bullet$ in $\mathfrak{t}$, as given in the description of $\Omega_{\infty}^{\mathrm{br}}$ above.) It is straightforward to check in this bijection that $\eta_{k}(\mathfrak{t})=\eta_{k}\left(\mathfrak{v}_{1}\right)+\eta_{k}\left(\mathfrak{t}_{1}^{\prime \prime}\right)+\cdots+\eta_{k}\left(\mathfrak{t}_{m}^{\prime \prime}\right)$, for $k \geq 0$, and that $\mathrm{wt}_{\infty}(\mathfrak{t})=j\left(H_{\delta}^{0} / r_{\delta}^{0} !\right) \mathrm{wt}_{0}\left(\mathfrak{t}_{1}^{\prime \prime}\right) \cdots \mathrm{wt}_{0}\left(\mathfrak{t}_{m}^{\prime \prime}\right)$. Then from the Product Lemma we obtain

$$
g_{j}=j \frac{1}{1 !} \sum \frac{H_{\delta}^{0}}{r_{\delta}^{0} !} \prod_{i \geq 1} \frac{f_{i}^{a_{i}}}{a_{i} !},
$$

since $\left[\left\{\mathfrak{v}_{1}\right\}, 1\right]_{\eta}=1 / 1$ !, and part (d) follows immediately from (14).

\section{Symmetrization AND A POLYNOMial TRANSFORMATiON}

At this stage, we have established the two generating series results that we need to prove Faber's Conjecture. The first of these results, on the degeneration side, is Corollary [3.9, the second form of the Degeneration Theorem, which gives a linear partial differential equation for the Faber-Hurwitz series $F^{g}$, in terms of the genus 0 Hurwitz series $\widehat{H}^{0}$. The second of these results, on the localization side, is Theorem 4.4, the second form of the Localization Tree Theorem, which expresses FaberHurwitz classes as a linear combination of the intersections $\left\langle\tau_{a_{1}} \cdots \tau_{a_{n}} \lambda_{k}\right\rangle_{g}^{\text {Fab }}$, in terms of $\widehat{H}^{0}$ and the genus 0 double Hurwitz series $H^{0}$.

In this section, in order to prove Faber's Intersection Number Conjecture, we introduce three operators, giving us a three-step transformation that will enable us to apply Corollary [3.9 and Theorem 4.4 conveniently, so that we can extract the intersection numbers of top degree, that are the subject of Faber's Conjecture. The first step is a symmetrization operator $\Xi_{m}$, and the second step is a change of variables $C$. The composition $C \Xi_{m}$ yields polynomials in the new variables, and the third step is the operator $\mathrm{T}^{\prime}$, that restricts to terms of maximum total degree. We shall refer to $\mathrm{T}^{\prime} \mathrm{C} \Xi_{m}=\mathrm{T} \Xi_{m}$ as the fundamental transformation.

Note that our fundamental transformation is only a slight modification of the three-step transformation that was used in GJV3 to give another proof of Getzler and Pandharipande's $\lambda_{g^{-}}$ Conjecture. There the transformation was applied to the Hurwitz number generating series in arbitrary genus, and the first two steps, of symmetrizing and changing variables, were identical. In that Hurwitz case, polynomiality also held, but the third step was to restrict to (full) terms of minimum total degree, so that the intersection numbers of bottom degree, that are the subject of the $\lambda_{g}$-Conjecture, could be extracted. Changes of variables for similar purposes arise in work of Kazarian and Lando [KL] and Shadrin and Zvonkine [SZ], as well as in [GJ2, GJVn, GJV1, GJV2]. 
5.1. Symmetrization. The first step of the fundamental transformation is the linear symmetrization operator $\Xi_{m}$.

Definition of $\Xi_{m}$ (Step 1): Following [GJV2], we define

$$
\Xi_{m}\left(p_{\alpha} z^{|\alpha|}\right):=\sum_{\sigma \in \mathfrak{S}_{m}} x_{\sigma(1)}^{\alpha_{1}} \cdots x_{\sigma(m)}^{\alpha_{m}}, \quad m \geq 1,
$$

if $l(\alpha)=m$ (with $\left.\alpha=\left(\alpha_{1}, \ldots, \alpha_{m}\right)\right)$, and zero otherwise.

In order to provide more compact expressions when applying $\Xi_{m}$, some notation is required. For $\rho=\left\{\rho_{1}, \ldots, \rho_{l}\right\} \subseteq\{1, \ldots, m\}$, let $x_{\rho}=\left\{x_{\rho_{1}}, \ldots, x_{\rho_{l}}\right\}$. Given $i_{1}, \ldots, i_{k} \geq 1$, with $i_{1}+\cdots+i_{k}=m$, let $s_{j}=i_{1}+\cdots+i_{j}$, for $j=1, \ldots, k$, and $s_{0}=0$. Also let

$$
\rho_{j}=\left\{s_{j-1}+1, \ldots, s_{j}\right\},
$$

for $j=1, \ldots, k$. Then $\operatorname{sym}_{i_{1}, \ldots, i_{k}}$ is a summation operator, over the set of ordered set partitions $\left(\nu_{1}, \ldots, \nu_{k}\right)$ of $\{1, \ldots, m\}$, in which, in the summand, $x_{\rho_{j}}$ is replaced by $x_{\nu_{j}}$, for $j=1, \ldots, k$.

We require the symmetrized series

$$
\left\{\begin{array}{llll}
F_{m}^{g}\left(x_{1}, \ldots, x_{m}\right) & :=\Xi_{m} F^{g}(z ; \mathbf{p}), & \widehat{\mathbf{H}}_{m}^{0}\left(x_{1}, \ldots, x_{m}\right) & :=\Xi_{m} \widehat{H}^{0}(z ; \mathbf{p}), \\
\mathbb{T}_{m}^{g}\left(x_{1}, \ldots, x_{m}, u\right) & :=\Xi_{m} \mathbb{T}^{g}(z, u ; \mathbf{p}), & \xi_{m}^{(i)}\left(x_{1}, \ldots, x_{m}, u\right) & \left.:=\Xi_{m} \xi^{(i)}(z, u ; \mathbf{p})\right),
\end{array}\right.
$$

for $m \geq 1$. When the arguments of these series are suppressed, they are the ones stated above.

5.2. Symmetrizing on the localization and degeneration sides. On the localization side, the following result is the third form of the Localization Tree Theorem. It is the symmetrized form of Theorem 4.4, and uses the substitution operator

$$
\Lambda: u \mapsto-u, x_{i} \mapsto(1-u)^{-1} x_{i}, \quad i=1, \ldots, m .
$$

Corollary 5.1 (Localization Tree Theorem - symmetrized functional equations). For $m, g \geq 1$,

$$
\text { a) } \quad F_{m}^{g} \mathbb{G}_{g, 1}=\left[u^{2 g-1}\right]\left(\frac{-u}{1-u}\right)^{m} \Lambda \mathbb{T}_{m}^{g}
$$

where

$$
\text { b) } \mathbb{T}_{m}^{g}=\sum_{n \geq 1} \frac{1}{n !} \sum_{\begin{array}{c}
a_{1}, \ldots, a_{n}, k \geq 0, \\
a_{1}+\cdots+a_{n}+k=g-2+n, \\
i_{1}, \ldots, i_{n} \geq 1, \\
i_{1}+\cdots+i_{n}=m
\end{array}}(-1)^{k}\left\langle\tau_{a_{1}} \cdots \tau_{a_{n}} \lambda_{k}\right\rangle_{g}^{\mathbb{F a b}} \text { sym }_{i_{1}, \ldots, i_{n}} \prod_{j=1}^{n} \xi_{i_{j}}^{\left(a_{j}\right)}\left(x_{\rho_{j}}\right) .
$$

Proof. The result follows by applying $\Xi_{m}$ to Theorem 4.4(a) and (b), for $m \geq 1$, and using the properties of $\Xi_{m}$ given in Lemmas 4.1-4.3 of GJVn.

On the degeneration side, the following result is the symmetrized form of Corollary [3.9, For each $1 \leq i<j \leq m$, note that there are two terms in the result with denominator $x_{i}-x_{j}$, and that the numerator is antisymmetric in $x_{i}, x_{j}$, so these terms combine to give a formal power series. To account for each individual term $\left(x_{i}-x_{j}\right)^{-1}$ that arises in the statement, we adopt the total ordering $x_{i} \prec x_{j}$ if $i<j$, and then define $\left(x_{i}-x_{j}\right)^{-1}$ by $\left(x_{i}-x_{j}\right)^{-1}:=x_{j}^{-1}\left(1-x_{i} x_{j}^{-1}\right)^{-1}$. This gives us an ordered Laurent series ring in $x_{1}, \ldots, x_{m}$.

Corollary 5.2 (Degeneration Theorem - symmetrized Join-cut Equation). For $m, g \geq 1$,

$$
\begin{aligned}
\left(\sum_{i=1}^{m} x_{i} \frac{\partial}{\partial x_{i}}+m-1\right) F_{m}^{g}= & \sum_{\substack{i_{2}, i_{3} \geq 0, i_{2}+i_{3}=m-1}} \operatorname{sym}_{1, i_{2}, i_{3}}\left(x_{1} \frac{\partial}{\partial x_{1}} \widehat{\mathbf{H}}_{1+i_{2}}^{0}\left(x_{1}, x_{\rho_{2}}\right)\right)\left(x_{1} \frac{\partial}{\partial x_{1}} F_{1+i_{3}}^{g}\left(x_{1}, x_{\rho_{3}}\right)\right) \\
& \quad+\operatorname{sym}_{1,1, m-2} \frac{x_{2}}{x_{1}-x_{2}} x_{1} \frac{\partial}{\partial x_{1}} F_{m-1}^{g}\left(x_{1}, x_{\rho_{3}}\right)+\sum_{i=1}^{m}\left(x_{i} \frac{\partial}{\partial x_{i}}\right)^{2 g+1} \widehat{\mathbf{H}}_{m}^{0} .
\end{aligned}
$$


Proof. The result follows by applying $\Xi_{m}$ to Corollary 3.9 for $m \geq 1$, and using the properties of $\Xi_{m}$ given in Lemmas 4.1-4.3 of GJVn.

Note that the second term on the right hand side of Corollary 5.2 vanishes in the case $m=1$.

5.3. Polynomiality and terms of top degree. The second step of the fundamental transformation is the change of variables $\mathrm{C}$.

Definition of C (Step 2): Let

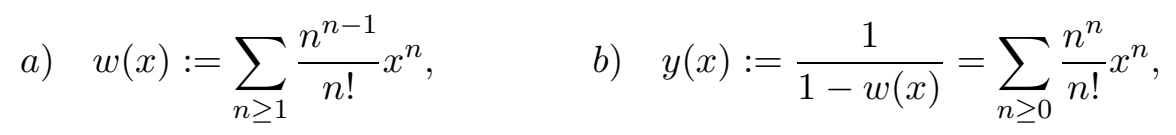

where the second equality in the expression for $y(x)$ is well-known (see, e.g., GJ2, Prop. 3.2.1]). Let $w_{i}:=w\left(x_{i}\right)$ and $y_{i}=y\left(x_{i}\right)$, for $i=1, \ldots, m$, and let $\mathrm{C}$ be an operator, applied to a formal power series in $x_{1}, \ldots, x_{m}$, that changes variables from the indeterminates $x_{1}, \ldots, x_{m}$ to $y_{1}, \ldots, y_{m}$. Thus, a direct way to apply $\mathrm{C}$ is to substitute $x_{i}=G\left(y_{i}-1\right)$, where $G(z)$ is the compositional inverse of the formal power series $\sum_{n \geq 1} n^{n} z^{n} / n$ !. In this paper, we do not apply this substitution directly to the symmetrized series $F_{m}^{g}, \Lambda \mathbb{T}_{m}^{g}, \Lambda \xi_{m}^{(i)}$, but instead quite indirectly. The details are intricate, but the key to our method is that in all cases the result is a polynomial in $y_{1}, \ldots, y_{m}$ (we say that a Laurent series in another indeterminate, either $u$ or $t$ in this paper, is polynomial if each of its coefficients is polynomial).

This key fact is recorded in the following result. The proof involves a number of transformations for implicitly defined series, and is deferred until Appendix A to avoid interrupting the present development. Note that part (b) follows from part (a) by Theorem 4.4(a),(b), and by (13) and Theorem 3.12 (which allows us to "divide" $\left\langle\tau_{a_{1}} \cdots \tau_{a_{n}} \lambda_{k}\right\rangle_{g}^{\mathbb{F a b}}$ by $\left.\mathbb{G}_{g, 1}\right)$. Consequently, in the Appendix it suffices to prove part (a) of Theorem 5.3 only.

Theorem 5.3. For $m=1,2,3$, we have
a) $\subset \Lambda \xi_{m}^{(i)} \in \mathbb{Q}\left[y_{1}, \ldots, y_{m}\right]((u)), \quad i \geq 1$
b) $\quad$ C $F_{m}^{g} \in \mathbb{Q}\left[y_{1}, \ldots, y_{m}\right], \quad g \geq 1$.

Theorem 5.3 is essential to our proof of Faber's Intersection Conjecture for at most 3 parts. In order for us to extend our proof to, say, $n$ parts, we would first need to prove Theorem 5.3 for all $m \leq n$. We are presently able to prove this result for $m \leq 5$, but not for larger values of $m$, since we require the symmetrized double Hurwitz series in our method of proof, and we only have explicit expressions for this when $m \leq 5$, as given in GJV2]. We conjecture that Theorem 5.3 holds for all positive integers $m$.

The third and final step of the fundamental transformation is the operator $\mathrm{T}^{\prime}$, that restricts a polynomial to terms of maximum total degree (these are referred to as the "top" terms).

Definition of $\mathrm{T}^{\prime}$ (Step 3): Let $\mathrm{S}_{i}$ be the operator that restricts a polynomial in $y_{1}, \ldots, y_{m}$ to the terms of total degree $i$. Then we define $\mathrm{T}^{\prime}$, when applied to $C F_{m}^{g}$, to denote $\mathrm{S}_{4 g+3 m-5}$. It turns out that there are no terms of higher degree in $C F_{m}^{g}$, so we say that $T^{\prime}$ restricts to the terms of top degree, though we understand that this is informal, since it assumes that the terms of total degree $4 g+3 m-5$ are not all zero.

If $\mathrm{C} \Lambda \xi_{m}^{(i)}=\sum_{k} a_{k} u^{k}$, we define $\mathrm{T}^{\prime} \mathrm{C} \Lambda \xi_{m}^{(i)}:=\sum_{k}\left(\mathrm{~S}_{2 g+2 m-2+k} a_{k}\right) u^{k}$. Again, it turns out that there are no terms is any of the $a_{k}$ of higher total degree, so in this case also $\mathrm{T}^{\prime}$ restricts to terms of top degree. We define $\mathrm{T}^{\prime}$, when applied to $\mathrm{C} \widehat{\mathbf{H}}_{m}^{0}$, to denote $\mathrm{S}_{3 m-6}$. Again there are no terms of higher total degree, so in this case also $\mathrm{T}^{\prime}$ restricts to terms of top degree.

Finally, in all other cases, we define $\mathrm{T}^{\prime}$ as a homomorphism, and we define $\mathrm{T}:=\mathrm{T}^{\prime} \mathrm{C}$.

5.4. Polynomiality and terms of top degree on the localization and degeneration sides. On the localization side, we can apply the fundamental transformation via Theorem 4.4, because 
of the polynomiality of $\Lambda \xi_{m}^{(i)}$ that was established in Theorem [5.3(a) for $m=1,2,3$. This requires the top terms, $\mathrm{T} \Lambda \xi_{m}^{(i)}$, which are given in the following result for $m=1,2,3$. Again, the proof is intricate, and is deferred until Appendix A to avoid interrupting the present development. The result uses the notation

$$
Y_{i}(u):=\frac{y_{i}}{1-u y_{i}}, \quad i \geq 1
$$

Theorem 5.4. For $i \geq 0$,

$$
\begin{aligned}
& \text { a) } u \mathrm{\top} \Lambda \xi_{1}^{(i)}=-(2 i-1) ! ! Y_{1}(u)^{2 i+1}, \quad \text { b) } \quad u^{2} \mathrm{\top} \Lambda \xi_{2}^{(i)}=-(2 i+1) ! ! u \operatorname{sym}_{1,1} \frac{y_{1}^{2} y_{2}}{y_{1}-y_{2}} Y_{1}(u)^{2 i+3} \\
& \text { c) } \frac{(-u)^{3} \mathrm{~T} \Lambda \xi_{3}^{(i)}}{(2 i+1) ! !}=u^{2} \operatorname{sym}_{1,1,1} \frac{y_{1}^{3} y_{2}^{4} y_{3}}{\left(y_{2}-y_{3}\right)\left(y_{1}-y_{2}\right)^{2}} Y_{1}(u)^{2 i+3} Y_{2}(u) \\
& -\operatorname{sym}_{1,2}\left(u y_{1} Y_{1}(u)^{2 i+5} Y_{2}(u) Y_{3}(u)-u^{2} y_{1}^{3} \frac{\partial}{\partial y_{1}} \frac{y_{1}^{3} y_{2} y_{3}}{\left(y_{2}-y_{1}\right)\left(y_{3}-y_{1}\right)} Y_{1}(u)^{2 i+4}\right) \text {. }
\end{aligned}
$$

On the degeneration side, we can apply the fundamental transformation via Corollary 5.2 if we can apply the change of variables $C$ to the partial differential operator $x_{i} \frac{\partial}{\partial x_{i}}$ and to the symmetrized generating series $\widehat{\mathbf{H}}_{m}^{0}$ for Hurwitz numbers, genus 0. This is straightforward in both cases.

For the partial differential operator, from $(33 \mathrm{3}, \mathrm{b})$ we have the functional equations $y_{i}=1 /\left(1-w_{i}\right)$, $y_{i}=1+x_{i} \frac{d w_{i}}{d x_{i}}$, and together these imply $x_{i} \frac{d y_{i}}{d x_{i}}=y_{i}^{2}\left(y_{i}-1\right)$. This immediately implies the operator identities

$$
\text { a) } \quad \mathrm{C} x_{i} \frac{\partial}{\partial x_{i}}=y_{i}^{2}\left(y_{i}-1\right) \frac{\partial}{\partial y_{i}} \mathrm{C}, \quad \text { b) } \quad \mathrm{T} x_{i} \frac{\partial}{\partial x_{i}}=y_{i}^{3} \frac{\partial}{\partial y_{i}} \mathrm{~T}
$$

For the symmetrized generating series $\widehat{\mathbf{H}}_{m}^{0}$, (11) and $(\underline{33 \mathrm{~b}} \mathrm{~b})$ give

$$
\widehat{\mathbf{H}}_{m}^{0}=\left(\sum_{j=1}^{m} x_{j} \frac{\partial}{\partial x_{j}}\right)^{m-3} \prod_{i=1}^{m}\left(y_{i}-1\right),
$$

for $m \geq 1$ (note that (35) applied to (36) immediately identifies $C \widehat{\mathbf{H}}_{m}^{0}$ as having degree $3 m-6$, consistent with the definition of $\mathrm{T}^{\prime}$ above). Also, we have

$$
\text { a) } x_{1} \frac{\partial}{\partial x_{1}} \widehat{\mathbf{H}}_{1}^{0}=1-y_{1}^{-1}, \quad \text { b) } \widehat{\mathbf{H}}_{2}^{0}=\log \left(\frac{y_{1}-y_{2}}{y_{1} y_{2}\left(x_{1}-x_{2}\right)}\right)+y_{1}^{-1}+y_{2}^{-1}-2,
$$

where the first of these expressions arises by applying $x_{1} \frac{d}{d x_{1}}$ to (36) when $m=1$, and using (351), and the second is given in [GJVn, p. 38].

\section{INTERSECTION NUMBERS With ONE PART}

In this section, we use our strategy for the first time. This involves applying the fundamental transformation to give results for the symmetrized Faber-Hurwitz series $F_{m}^{g}$ on both the degeneration and localization sides, to obtain results for Faber's intersection numbers. Here, these results are given in Section 6.1 for the case of a single $(m=1)$ part. Faber's Intersection Number Conjecture, giving $\left\langle\tau_{g-1}\right\rangle_{g}^{\mathbb{F a b}}=\psi_{1}^{g-1}$, is immediate in this case, but it gives us an equation relating the two generators $\psi_{1}^{g-1}$ and $\mathbb{G}_{g, 1}$ of $A_{2 g-1}\left(\mathcal{M}_{g, n}^{r t}\right)$. There is non-trivial geometric information to be gained by relating these generators, which is described in Section 6.2. 
6.1. Intersection numbers with one part. We begin with a result for the localization side.

Theorem 6.1. For $g \geq 1$, we have $\mathrm{T} F_{1}^{g}\left(x_{1}\right) \mathbb{G}_{g, 1}=(2 g-3) ! !\left(\begin{array}{c}4 g-3 \\ 2 g-1\end{array}\right) y_{1}^{4 g-2} \psi_{1}^{g-1}$.

Proof. From Corollary 5.1(b) we have $\mathbb{T}_{1}^{g}=\sum_{k=0}^{g-1}(-1)^{k}\left\langle\tau_{g-1-k} \lambda_{k}\right\rangle_{g}^{\mathbb{F a b}} \xi_{1}^{(g-1-k)}$. Together with Theorem 5.4 (a), this gives

$\left[u^{2 g-1}\right](-u)^{1} \mathrm{~T} \Lambda \mathbb{T}_{1}^{g}\left(x_{1}, u\right)=\left[u^{2 g-1}\right]\left\langle\tau_{g-1}\right\rangle_{g}^{\mathbb{F a b}}(2 g-3) ! ! Y_{1}(u)^{2 g-1}=\left[u^{2 g-1}\right](2 g-3) ! ! Y_{1}(u)^{2 g-1} \psi_{1}^{g-1}$

where, for the second equality, we have used the immediate fact that $\left\langle\tau_{g-1}\right\rangle_{g}^{\mathbb{F a b}}=\psi_{1}^{g-1}$ for $g \geq 1$. But $Y_{1}(u)^{2 g-1}=\sum_{i \geq 0}\left(\begin{array}{c}(2 g-1) \\ i\end{array}\right)(-1)^{i} y_{1}^{2 g-1+i} u^{i}=\sum_{i \geq 0}\left(\begin{array}{c}2 g-2+i \\ i\end{array}\right) y_{1}^{2 g-1+i} u^{i}$, and the result follows immediately.

Now we turn to the corresponding result for the degeneration side.

Theorem 6.2. For $g \geq 1$, we have $\mathrm{T} F_{1}^{g}\left(x_{1}\right)=(4 g-3) ! ! \frac{y_{1}^{4 g-2}}{4 g-2}$.

Proof. Let $m=1$ in Corollary [5.2, to obtain $x_{1} \frac{\partial}{\partial x_{1}} F_{1}^{g}=\left(x_{1} \frac{\partial}{\partial x_{1}} \widehat{\mathbf{H}}_{1}^{0}\right)\left(x_{1} \frac{\partial}{\partial x_{1}} F_{1}^{g}\right)+\left(x_{1} \frac{\partial}{\partial x_{1}}\right)^{2 g+1} \widehat{\mathbf{H}}_{1}^{0}$. Solving for $x_{1} \frac{\partial}{\partial x_{1}} F_{1}^{g}$, and using (37a), we obtain

$$
x_{1} \frac{\partial}{\partial x_{1}} F_{1}^{g}=y_{1}\left(x_{1} \frac{\partial}{\partial x_{1}}\right)^{2 g-1}\left(y_{1}-1\right) .
$$

The polynomiality of $F_{1}^{g}$ follows immediately from (35) (or we can use Theorem 5.3(b), with $m=1$ ), so from (35b) we have $y_{1}^{3} \frac{\partial}{\partial y_{1}} \top F_{1}^{g}=y_{1}\left(y_{1}^{3} \frac{\partial}{\partial y_{1}}\right)^{2 g-1} y_{1}=(4 g-3) ! ! y_{1}^{4 g}$, where the second equality follows by induction. The result follows by integrating in $y_{1}$.

6.2. Some immediate geometric consequences. Comparing the above two results, we obtain the following result, which relates the generator $\mathbb{G}_{g, 1}$ of $A_{2 g-1}\left(\mathcal{M}_{g, n}^{r t}\right)$ with the generator $\psi_{1}^{g-1}$ suggested by Faber's Intersection Number Conjecture.

Corollary 6.3. For $g \geq 1, \mathbb{G}_{g, 1}=\frac{2^{g}}{(g-1) !} \psi_{1}^{g-1}$ on $A_{2 g-1}\left(\mathcal{M}_{g, 1}\right)$.

In particular, $\psi_{1}^{g-1}=\left\langle\tau_{g-1}\right\rangle_{g}^{\mathbb{F a b}}$ is another non-zero element (=basis) of the one-dimensional vector space $A_{2 g-1}\left(\mathcal{M}_{g, 1}\right)$, and this describes the change of basis from $\mathbb{G}_{g, 1}$ to $\psi_{1}^{g-1}$.

Proof. Comparing Theorems 6.1 and 6.2 , we obtain $(2 g-3) ! !\left(\begin{array}{l}4 g-3 \\ 2 g-1\end{array}\right) \psi_{1}^{g-1}=\frac{(4 g-3) ! !}{4 g-2} \mathbb{G}_{g, 1}$, and the result follows immediately.

Corollary 6.4 ([1, Thm. 4]). $\quad \sum_{i=0}^{g-2}(-1)^{i} \lambda_{i} \psi_{1}^{g-1-i}=2^{g-1} \psi_{1}^{g-1} / g$ !

Proof. Applying the usual (non-relative) virtual localization formula to $\mathbb{G}_{g, 1, \sim}$, we obtain $\mathbb{G}_{g, 1, \sim}=$ $\psi_{1}^{2 g-1}-\psi_{1}^{2 g-2} \lambda_{1}+\cdots+(-1)^{g} \psi_{1}^{g-1} \lambda_{g}$. The result then follows from Corollary 6.3 and the fact that $\mathbb{G}_{g, 1}=2 g \mathbb{G}_{g, 1, \sim}$ (Proposition 3.1).

Corollary 6.5. The class of the hyperelliptic curves in $\mathcal{M}_{g}$ is $\frac{\left(2^{2 g}-1\right) 2^{g-1}}{g !(2 g+1)(2 g+2)} \kappa_{g-2}$.

(This was stated, for example, in the concluding remarks of [F1].) The argument carries through for the locus of degree $d$ covers fully ramified over two points.

Sketch of proof. The class $\mathbb{G}_{g, 2, \sim}$ corresponds to the points $[C, p, q] \in \mathcal{M}_{g, 2}^{r t}$ where $\mathcal{O}_{C}(p-q)$ is a 2 -torsion point (along with a virtual class). The locus where $\mathcal{O}_{C}(p-q)$ is trivial corresponds to $\mathbb{G}_{g, 1}$. Thus the locus where $\mathcal{O}_{C}(p-q)$ is 2 -torsion but non-trivial has virtual class $\left(2^{2 g}-1\right) \mathbb{G}_{g, 1}$ 
by Theorem 3.5. It is straightforward to check the virtual fundamental class on this locus is the actual fundamental class (as mentioned in the proof of Theorem 3.5). This locus corresponds to hyperelliptic curves with two marked Weierstrass points. Thus the locus in $\mathcal{M}_{g, 1}$ of hyperelliptic curves with choice of one Weierstrass point has class $\frac{2^{2 g}-1}{2 g+1} \mathbb{G}_{g, 1}=\frac{\left(2^{2 g}-1\right) 2^{g-1}}{g !(2 g+1)} \psi_{1}^{g-1}$. Pushing forward to $\mathcal{M}_{g}$, and forgetting the choice of one of the $2 g+2$ Weierstrass points, we get the desired result.

Corollary 6.6. For $d, g \geq 1$, we have $F_{(d)}^{g}=\frac{1}{d} \sum_{i=1}^{d}\left(\begin{array}{c}d \\ i\end{array}\right) i^{2 g+i-1}(d-i)^{d-i}$.

This is a corollary of the proof of Theorem 6.2, not of the result itself.

Proof. From (38) and (33b), we have $x_{1} \frac{\partial}{\partial x_{1}} F_{1}^{g}=\sum_{n \geq 0} \frac{n^{n}}{n !} x_{1}^{n} \sum_{i \geq 1} \frac{i^{2 g+i-1}}{i !} x_{1}^{i}$, and the result follows from (31) and (15).

Thus, for example, the class of non-singular genus $g$ curves $C$ admitting a degree $d$ cover of $\mathbb{P}^{1}$ via $\mathcal{O}(d p)$ for some $p \in C$, ramified over an appropriate number of points is

$$
\frac{1}{d} \sum_{i=1}^{d}\left(\begin{array}{l}
d \\
i
\end{array}\right) i^{2 g+i-1}(d-i)^{d-i} \mathbb{G}_{g, 1}=\frac{1}{d} \sum_{i=1}^{d}\left(\begin{array}{l}
d \\
i
\end{array}\right) i^{2 g+i-1}(d-i)^{d-i} \frac{2^{g} \kappa_{g-2}}{(g-1) !} \in A_{2 g-1}\left(\mathcal{M}_{g}\right) .
$$

\section{A strategy for an inductive proof of Faber's Intersection Number Conjecture}

In this section, we consider an inductive strategy for proving the Conjecture.

7.1. Final form of the Localization Tree Theorem. First, we define two generating series, in which the genus $g$ is marked by the indeterminate $t$.

The localization tree generating series is

(39) $\Psi_{m}\left(y_{1}, \ldots, y_{m}, t\right):=\sum_{g \geq 1} \frac{t^{2 g}}{(2 g-1) ! !}\left[u^{2 g-1}\right](-u)^{m} \mathrm{\top} \Lambda \mathbb{T}_{m}^{g}\left(x_{1}, \ldots, x_{m}, u\right) / \psi_{1}^{g-1}, \quad m \geq 1$.

The Faber generating series is

$$
\Phi_{m}\left(y_{1}, \ldots, y_{m}, t\right):=\sum_{g \geq 1} 2^{2 g-1} \frac{t^{2 g}}{(2 g-1) !} \mathrm{\top} F_{m}^{g}\left(x_{1}, \ldots, x_{m}\right), \quad m \geq 1 .
$$

For $\Psi_{m}$, thanks to the fact that $R_{2 g-1}\left(\mathcal{M}_{g, 1}\right) \cong \mathbb{Q}$ (Thm. 3.12), we "divide by $\psi_{1}^{g-1 "}$ " using the isomorphism

$$
\mathbb{Q} \stackrel{\times \psi_{1}^{g-1}}{\longrightarrow} R_{2 g-1}\left(\mathcal{M}_{g, 1}\right)
$$

(combining (23) and Cor. 6.3), and thus we define the Faber intersection number

$$
\langle\cdots\rangle_{g}^{\mathrm{Fab}}:=\langle\cdots\rangle_{g}^{\mathbb{F a b}} / \psi_{1}^{g-1}=\langle\cdots\rangle_{g}^{\mathbb{F a b}} /\left\langle\tau_{g-1}\right\rangle_{g}^{\mathbb{F a b}} .
$$

Note that $\Psi_{m}$ and $\Phi_{m}$, since they require the operator T, are only defined when the operator C yields a polynomial. So far, we have proved this polynomiality only for $m=1,2,3$, in Theorem 5.3 , Subject to this, $\Psi_{m}$ and $\Phi_{m}$ are both generating series with rational coefficients, and the next result shows that they are equal.

Corollary 7.1 (Localization Tree Theorem — for genus generating series). For $m \geq 1$,

$$
\Psi_{m}\left(y_{1}, \ldots, y_{m}, t\right)=\Phi_{m}\left(y_{1}, \ldots, y_{m}, t\right) .
$$


Proof. The result follows from Corollary $5.1($ a $)$ by noting that $\frac{\mathbb{G}_{g, 1}}{(2 g-1) ! !}=\frac{2^{g} \psi_{1}^{g-1}}{(g-1) !(2 g-1) ! !}=\frac{2^{2 g-1} \psi_{1}^{g-1}}{(2 g-1) !}$ for $g \geq 1$, from Corollary 6.3 ,

Corollary 7.1 is the final form of Theorem 3.11. It summarizes the relationship between the localization side (dealing with $\Psi_{m}$ ) and the degeneration side (dealing with $\Phi_{m}$ ) as the equality of $\Psi_{m}$ and $\Phi_{m}$. Our strategy for exploiting this relationship in order to prove the Faber Conjecture for a fixed number of parts is indirect. We prove in the following lemma that Corollary 7.1 implies sufficiently many linearly independent linear equations for Faber's intersection numbers to uniquely identify them. This means that we can prove the Faber Conjecture iteratively on the number of parts, by simply verifying the equality $\Psi_{m}=\Phi_{m}$ of Corollary 7.1 with Faber's conjectured values substituted for the intersection numbers. In fact, as we shall see, with these substituted values, we are able to verify the equality $\Psi_{m}=\Phi_{m}$ for particular $m$ simply by equating polynomials.

Lemma 7.2. For any $n \geq 2$, if the Faber Conjecture is true for up to $n-1$ parts, and

$$
\Psi_{n}\left(y_{1}, \ldots, y_{n}, t\right)=\Phi_{n}\left(y_{1}, \ldots, y_{n}, t\right)
$$

with Faber's conjectured values substituted for the intersection numbers with up to $n$ parts, then the Faber Conjecture is true for up to $n$ parts.

Proof. Consider the equality $\Psi_{n}\left(y_{1}, \ldots, y_{n}, t\right)=\Phi_{n}\left(y_{1}, \ldots, y_{n}, t\right)$, and equate coefficients of $t^{2 g}$ for each fixed $g \geq 2$. This gives an equation involving Faber's intersection numbers with at most $n$ parts, and from Corollary 5.1(b), (39) and Theorem5.4(a), we deduce that the only terms involving the intersection numbers with exactly $n$, positive, parts are given by

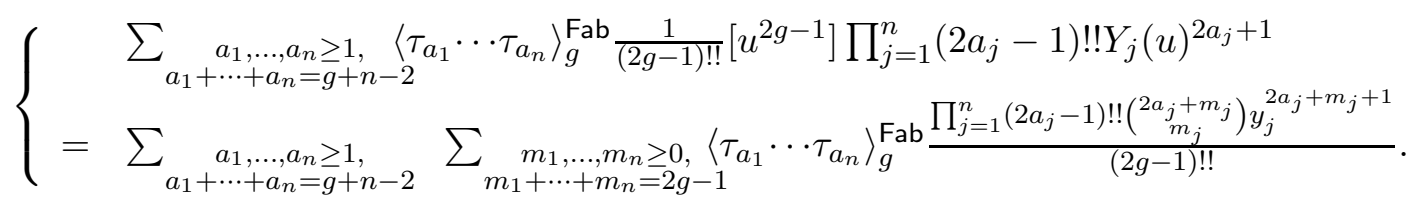

Now under the hypothesis that the Faber Conjecture has been proved for up to $n-1$ parts, we are able to evaluate all Faber intersection numbers in the above equation with up to $n-1$ parts, as well as those with $n$ parts, at least one of which is zero (by use of the string equation), and we consider Faber's intersection numbers with exactly $n$, positive, parts as unknowns. Now equate coefficients of $y_{1}^{i_{1}} \cdots y_{n}^{i_{n}}$ in this equation for each $i_{1}, \ldots, i_{n} \geq 3$ with $i_{1}+\cdots+i_{n}=4 g-5+3 n$, to obtain a linear equation that we shall refer to as $B_{i_{1}, \ldots, i_{n}}$. Then we thus obtain a system of $\left(\begin{array}{c}4 g-6+n \\ n-1\end{array}\right)$ equations $B_{i_{1}, \ldots, i_{n}}$ in the $\left(\begin{array}{c}g-3+n \\ n-1\end{array}\right)$ "unknowns" $\left\langle\tau_{a_{1}} \cdots \tau_{a_{n}}\right\rangle_{g}^{\text {Fab }}$, where $a_{1}, \ldots, a_{n} \geq 1$, with $a_{1}+\cdots+a_{n}=g-2+n$. Let the coefficient matrix for this system be denoted by $\mathbf{M}$, and note that $\left(\begin{array}{c}4 g-6+n \\ n-1\end{array}\right) \geq\left(\begin{array}{c}g-3+n \\ n-1\end{array}\right)$, since $g, n \geq 2$. (We will not use the symmetry of these unknowns.)

Now we prove that this system has at most one solution. To do so, suppose that we consider the unknowns in lexicographic order of the $a_{1} \ldots a_{n}$, and the equations in lexicographic order of the $i_{1} \ldots i_{n}$ (so we have ordered the rows and columns of M.) Note that, from (42), the entry in row $i_{1} \ldots i_{n}$ and column $a_{1} \ldots a_{n}$ is non-zero if and only if $i_{j} \geq 2 a_{j}+1$ for all $j=1, \ldots, n$. Then it is straightforward to check that the submatrix of $\mathbf{M}$ consisting of all columns, and rows $i_{1}, \ldots, i_{n}$ with $i_{1}, \ldots, i_{n-1} \geq 3$ and odd, and $i_{1}+\cdots+i_{n-1} \leq 2 g-7+3 n$, is lower triangular, with non-zero coefficients on the diagonal. Thus these rows of $\mathbf{M}$ are linearly independent, so the system has at most one solution. The result follows since, if Faber's conjectured values satisfy this system, then they are uniquely the correct values for these intersection numbers.

7.2. A lemma for the localization tree generating series. We consider first the localization side. In order to determine a compact rational expression for the localization tree generating series $\Psi_{m}$, we shall need the following lemma, where $\mathrm{E} f$ denotes the even subseries of the formal power 
series $f$ in the indeterminate $t$. Also, for $i \geq 1$ we let

$$
B_{i}:=\sqrt{1-4 y_{i}^{2} t}, \quad A_{i}:=\frac{1-B_{i}}{2 y_{i}} .
$$

Lemma 7.3. For a formal power series $f, \sum_{g \geq 1} t^{2 g}\left[u^{2 g-1}\right] f(u) Y_{1}(u)^{2 g-1}=\frac{1}{2} \mathrm{E} t\left(1+B_{1}^{-1}\right) f\left(A_{1}\right)$.

Proof. Now $\left[u^{2 g-1}\right] f(u) Y_{1}(u)^{2 g-1}=\left[t^{2 g-1}\right] \frac{f(s)}{1-t \frac{d}{d s} Y_{1}(s)}$, from Lagrange's Implicit Function Theorem (see, e.g., GJ1, Thm. 1.2.4]), where $s$ is the unique formal power series solution (in $t$ ) of $s=t Y_{1}(s)$, which is the equation $y_{1} s^{2}-s+y_{1} t=0$, and so $s=A_{1}$. But $\frac{d}{d s} Y_{1}(s)=Y_{1}(s)^{2}$, so with $s=A_{1}$, we have $1-t \frac{d}{d s} Y_{1}(s)=\frac{2 B_{1}}{1+B_{1}}$. Then $\left[u^{2 g-1}\right] f(u) Y_{1}(u)^{2 g-1}=\left[t^{2 g-1}\right] \frac{1}{2}\left(1+B_{1}^{-1}\right) f\left(A_{1}\right)$. The result follows.

7.3. Final form of the Degeneration Theorem. For the degeneration side, we describe the general results that will allow us to obtain an explicit rational expression for the Faber generating series $\Phi_{m}$. First we give a compact expression for $\Phi_{1}$.

Corollary 7.4. $\quad y_{1} \frac{\partial}{\partial y_{1}} \Phi_{1}\left(y_{1}, t\right)=\mathrm{E} t B_{1}^{-1}$.

Proof. From (40) with $m=1$, and Theorem 6.2, we obtain

$$
y_{1} \frac{\partial}{\partial y_{1}} \Phi_{1}\left(y_{1}, t\right)=\sum_{g \geq 1} 2^{2 g-1} \frac{(4 g-3) ! !}{(2 g-1) !} y_{1}^{4 g-2} t^{2 g}=t \sum_{g \geq 1}\left(\begin{array}{c}
-\frac{1}{2} \\
2 g-1
\end{array}\right)\left(-4 y_{1}^{2} t\right)^{2 g-1},
$$

and the result follows immediately.

In the next result, we consider the top terms in Corollary [5.2, to obtain a simple iterative equation for the Faber generating series $\Phi_{m}, m \geq 2$. Similarly to Corollary 5.2, there are two terms in this result with denominator $y_{i}-y_{j}$, and the numerator is antisymmetric in $y_{i}, y_{j}$, so these terms combine to give a formal power series. Also, to account for each individual term $\left(y_{i}-y_{j}\right)^{-1}$, we adopt the total ordering $y_{i} \prec y_{j}$ if $i<j$, to obtain an ordered Laurent series ring in $y_{1}, \ldots, y_{m}$.

The statement of the result uses the operator

$$
\Delta_{k}:=\sum_{i \geq 1} y_{i}^{k} \frac{\partial}{\partial y_{i}}, \quad k \geq 2
$$

and the generating series

$$
\Upsilon_{m}\left(y_{1}, \ldots, y_{m}, t\right):=\sum_{g \geq 1} 2^{2 g-1} \frac{t^{2 g}}{(2 g-1) !} \top \sum_{i=1}^{m}\left(x_{i} \frac{\partial}{\partial x_{i}}\right)^{2 g+1} \widehat{\mathbf{H}}_{m}^{0}\left(x_{1}, \ldots, x_{m}\right), \quad m \geq 1 .
$$

Lemma 7.5 (Degeneration Theorem - Join-cut equation for $\Phi_{m}$ ). For $m \geq 2$ we have

$$
\begin{aligned}
\Delta_{2} \Phi_{m}\left(y_{1}, \ldots, y_{m}, t\right)= & \sum_{\substack{i_{2} \geq 2, i_{3} \geq 0, i_{2}+i_{3}=m-1}} \operatorname{sym}_{1, i_{2}, i_{3}}\left(y_{1}^{3} \frac{\partial}{\partial y_{1}} \mathrm{~T} \widehat{\mathbf{H}}_{1+i_{2}}^{0}\left(x_{1}, x_{\rho_{2}}\right)\right)\left(y_{1}^{3} \frac{\partial}{\partial y_{1}} \Phi_{1+i_{3}}\left(y_{1}, y_{\rho_{3}}, t\right)\right) \\
& +\operatorname{sym}_{1,1, m-2} \frac{y_{1}^{5} y_{2}}{y_{1}-y_{2}} \frac{\partial}{\partial y_{1}} \Phi_{m-1}\left(y_{1}, y_{\rho_{3}}, t\right)+\Upsilon_{m}\left(y_{1}, \ldots, y_{m}, t\right) .
\end{aligned}
$$

Proof. From the symmetrized Join-cut equation (Cor. [5.2), and moving the contribution for $i_{2}=0$ from the summation on the right hand side to the left hand side, and using (37a) and (35) a), we have $\left(\sum_{i=1}^{m} \frac{x_{i}}{y_{i}} \frac{\partial}{\partial x_{i}}+m-1\right) F_{m}^{g}$ on the left hand side. But, from (37b) and (35a), we have

$$
x_{1} \frac{\partial}{\partial x_{1}} \widehat{\mathbf{H}}_{2}^{0}=\frac{y_{1}^{2}\left(y_{1}-1\right)}{y_{1}-y_{2}}-y_{1}\left(y_{1}-1\right)-\left(y_{1}-1\right)-\frac{x_{1}}{x_{1}-x_{2}}=\frac{y_{1}^{2}\left(y_{2}-1\right)}{y_{1}-y_{2}}-\frac{x_{2}}{x_{1}-x_{2}},
$$


and we apply this result to combine the contribution for $i_{2}=1$ in the summation on the right hand side with the sym $_{1,1, m-2}$ term on the right hand side, to give

$$
\begin{aligned}
& \sum_{\substack{i_{2} \geq 2, i_{3} \geq 0, i_{2}+i_{3}=m-1}} \operatorname{sym}_{1, i_{2}, i_{3}}\left(x_{1} \frac{\partial}{\partial x_{1}} \widehat{\mathbf{H}}_{1+i_{2}}^{0}\left(x_{1}, x_{\rho_{2}}\right)\right)\left(x_{1} \frac{\partial}{\partial x_{1}} F_{1+i_{3}}^{g}\left(x_{1}, x_{\rho_{3}}\right)\right) \\
& +\operatorname{sym}_{1,1, m-2} \frac{y_{1}^{2}\left(y_{2}-1\right)}{y_{1}-y_{2}} x_{1} \frac{\partial}{\partial x_{1}} F_{m-1}^{g}\left(x_{1}, x_{\rho_{3}}\right)+\sum_{i=1}^{m}\left(x_{i} \frac{\partial}{\partial x_{i}}\right)^{2 g+1} \widehat{\mathbf{H}}_{m}^{0},
\end{aligned}
$$

on the right hand side. Now, multiply on both sides by $2^{2 g-1} \frac{t^{2 g}}{(2 g-1) !}$, sum over $g \geq 1$, and apply T, to obtain the result, from (40) and (35b).

Lemma 7.5 is the final form of Theorem 3.7. In order to apply this result, we require a technical result about the invertibility of the partial differential operator $\Delta_{k}$.

Proposition 7.6. For $m \geq 1, k \geq 2$, the operator $\Delta_{k}$ is invertible for formal power series in $y_{1}, \ldots, y_{m}$ in which every monomial has positive exponent for each of $y_{1}, \ldots, y_{m}$.

Proof. Let $A:=\sum_{n_{1}, \ldots, n_{m} \geq 1} a_{\left(n_{1}, \ldots, n_{m}\right)} y_{1}^{n_{1}} \cdots y_{m}^{n_{m}}$ and $B:=\sum_{n_{1}, \ldots, n_{m} \geq 1} b_{\left(n_{1}, \ldots, n_{m}\right)} y_{1}^{n_{1}} \cdots y_{m}^{n_{m}}$, and suppose that $\Delta_{k} A=\Delta_{k} B$. We prove that $a_{\left(n_{1}, \ldots, n_{m}\right)}=b_{\left(n_{1}, \ldots, n_{m}\right)}$ for all $n_{1}, \ldots, n_{m} \geq 1$ by putting a partial order $\prec_{\ell}$ on the $\left(n_{1}, \ldots, n_{m}\right)$ : we let $\left(n_{1}, \ldots, n_{m}\right) \leq$ be the vector of length $m$ containing the $n_{i}$ in weakly increasing order, and we define $\left(n_{1}, \ldots, n_{m}\right) \prec_{\ell}\left(i_{1}, \ldots, i_{m}\right)$ if and only if $\left(n_{1}, \ldots, n_{m}\right) \leq$ precedes $\left(i_{1}, \ldots, i_{m}\right) \leq$ in lexicographic order.

To prove the result for $\left(n_{1}, \ldots, n_{m}\right)$, suppose that $n_{i}=\max \left\{n_{1}, \ldots, n_{m}\right\}$ (make an arbitrary choice of $i$ if there is more than one, equal, maximum element), and equate coefficients of $y_{1}^{n_{1}} \cdots y_{i}^{n_{i}+k-1} \cdots y_{m}^{n_{m}}$ in $\Delta_{k} A=\Delta_{k} B$, to obtain

$$
\left\{\begin{aligned}
& n_{i} a_{\left(n_{1}, \ldots, n_{m}\right)}+\sum_{\substack{j \neq i, n_{j} \geq k}}\left(n_{j}-k+1\right) a_{\left(n_{1}, \ldots, n_{j}-k+1, \ldots, n_{i}+k-1, \ldots, n_{m}\right)} \\
= & n_{i} b_{\left(n_{1}, \ldots, n_{m}\right)}+\sum_{\substack{j \neq i, n_{j} \geq k}}\left(n_{j}-k+1\right) b_{\left(n_{1}, \ldots, n_{j}-k+1, \ldots, n_{i}+k-1, \ldots, n_{m}\right)} .
\end{aligned}\right.
$$

Now consider any fixed linear extension of $\prec_{\ell}$, and note that, for any term in the summations in (46), we have $\left(n_{1}, \ldots, n_{j}-k+1, \ldots, n_{i}+k-1, \ldots, n_{m}\right) \prec_{\ell}\left(n_{1}, \ldots, n_{m}\right)$. The result follows from (46), by induction on the position in this linear extension.

\section{Proof of Faber's Intersection Number Conjecture for 2 and 3 Parts, with COMMENTS ABOUT THE GENERAL CASE OF $m$ PARTS}

8.1. Faber's Intersection Number Conjecture for two parts. In this section, we prove Faber's Conjecture for the case of two parts. It will be convenient to "smooth" the result to allow zeros, by applying the string equation. For cases of (2) when some $d_{i}=0$, we can use the string equation to deduce a consistent value. For example, when $n=2, d_{1}=g, d_{2}=0$, the string equation gives $\left\langle\tau_{g} \tau_{0}\right\rangle_{g}^{\mathrm{Fab}}=\left\langle\tau_{g-1}\right\rangle_{g}^{\mathrm{Fab}}=1$, which agrees with (2), so Faber's Conjecture for $n=2$ becomes

$$
\left\langle\tau_{d_{1}} \tau_{d_{2}}\right\rangle_{g}^{\mathrm{Fab}}=\frac{(2 g-1) ! !}{\left(2 d_{1}-1\right) ! !\left(2 d_{2}-1\right) ! !}, \quad d_{1}+d_{2}=g, \quad d_{1}, d_{2} \geq 0, \quad g \geq 1 .
$$

For the localization side, we have the following result. In the proof, we use the notation

$$
\widehat{Y}_{m}:=Y_{m}\left(A_{1}\right), \quad m \geq 1,
$$

where $Y_{m}, A_{1}$ are defined in (43), (34), respectively. 
Theorem 8.1. If the Faber Conjecture is true for $n=2$, then

$$
\Psi_{2}\left(y_{1}, y_{2}, t\right)=\operatorname{Esym}_{1,1}\left(\frac{y_{1}^{3} y_{2} t}{y_{1}-y_{2}}-\frac{y_{1}^{4} y_{2}^{2} t}{\left(y_{1}-y_{2}\right)\left(y_{1}^{2}-y_{2}^{2}+4 y_{1}^{2} y_{2}^{2} t\right)}\right) B_{1}^{-1} \text {. }
$$

Proof. From Corollary [5.1(b) we have $\mathbb{T}_{2}^{g}=\sum_{\substack{a_{1}, a_{2} \geq 0, a_{1}+a_{2} \leq g}}(-1)^{g-a_{1}-a_{2}}\left\langle\tau_{a_{1}} \tau_{a_{2}} \lambda_{g-a_{1}-a_{2}}\right\rangle_{g}^{\mathbb{F a b}} \xi_{1}^{\left(a_{1}\right)}\left(x_{1}\right)$ $\xi_{1}^{\left(a_{2}\right)}\left(x_{2}\right)+\sum_{k=0}^{g-1}(-1)^{k}\left\langle\tau_{g-1-k} \lambda_{k}\right\rangle_{g}^{\mathbb{F a b}} \xi_{2}^{(g-1-k)}$, where we have used the symmetry of the Faber symbol in the first summation. Together with Theorem 5.4(a),(b) and (39), this gives

$$
\begin{aligned}
\Psi_{2}\left(y_{1}, y_{2}, t\right)= & \sum_{g \geq 1} \frac{t^{2 g}}{(2 g-1) ! !}\left[u^{2 g-1}\right] \sum_{\begin{array}{c}
a_{1}, a_{2} \geq 0, \\
a_{1}+a_{2}=g
\end{array}}\left\langle\tau_{a_{1}} \tau_{a_{2}}\right\rangle_{g}^{\mathrm{Fab}} \prod_{j=1}^{2}\left(2 a_{j}-1\right) ! ! Y_{j}(u)^{2 a_{j}+1} \\
& -\sum_{g \geq 1} \frac{t^{2 g}}{(2 g-1) ! !}\left[u^{2 g-1}\right]\left\langle\tau_{g-1}\right\rangle_{g}^{\mathrm{Fab}}(2 g-1) ! ! u \text { sym }_{1,1} \frac{y_{1}^{2} y_{2}}{y_{1}-y_{2}} Y_{1}(u)^{2 g+1} .
\end{aligned}
$$

Now, if the Faber Conjecture is true for $n=2$, then from (47), we have

$$
\Psi_{2}\left(y_{1}, y_{2}, t\right)=\sum_{g \geq 1} t^{2 g}\left[u^{2 g-1}\right]\left(\sum_{\substack{a_{1}, a_{2} \geq 0, a_{1}+a_{2}=g}} Y_{1}(u)^{2 a_{1}+1} Y_{2}(u)^{2 a_{2}+1}-\operatorname{sym}_{1,1} \frac{y_{1}^{2} y_{2}}{y_{1}-y_{2}} u Y_{1}(u)^{2 g+1}\right),
$$

But $\sum_{\substack{a_{1}, a_{2} \geq 0, a_{1}+a_{2}=g}}, Y_{1}(u)^{2 a_{1}+1} Y_{2}(u)^{2 a_{2}+1}=\operatorname{sym}_{1,1} \frac{Y_{1}(u)^{2 g+3} Y_{2}(u)}{Y_{1}(u)^{2}-Y_{2}(u)^{2}}$, and from Lemma 7.3 we conclude that

$$
\Psi_{2}\left(y_{1}, y_{2}, t\right)=\frac{1}{2} \operatorname{Esym}_{1,1} t\left(1+B_{1}^{-1}\right)\left(\frac{\widehat{Y}_{1}^{4} \widehat{Y}_{2}}{\widehat{Y}_{1}^{2}-\widehat{Y}_{2}^{2}}-\frac{y_{1}^{2} y_{2}}{y_{1}-y_{2}} A_{1} \widehat{Y}_{1}^{2}\right) .
$$

From the proof of Lemma 7.3, we have $A_{1}=t y_{1} /\left(1-y_{1} A_{1}\right)$, so routinely simplifying, we obtain

$$
\begin{aligned}
\Psi_{2}\left(y_{1}, y_{2}, t\right) & =\operatorname{Esym}_{1,1}\left(\frac{y_{1}^{3} y_{2} t A_{1}\left(1-y_{2} A_{1}\right) B_{1}^{-1}}{\left(y_{1}-y_{2}\right)\left(y_{1}+y_{2} B_{1}\right)}-\frac{y_{1}^{3} y_{2} A_{1}^{2} B_{1}^{-1}}{y_{1}-y_{2}}\right) \\
& =\operatorname{Esym}_{1,1}\left(\frac{y_{1}^{3} y_{2} t B_{1}^{-1}}{y_{1}-y_{2}}+\frac{y_{1}^{3} y_{2}^{3} t-y_{1}^{4} y_{2}^{2} t B_{1}^{-1}}{\left(y_{1}-y_{2}\right)\left(y_{1}^{2}-y_{2}^{2} B_{1}^{2}\right)}\right) .
\end{aligned}
$$

Now it is straightforward to verify that $\mathrm{Esym}_{1,1} \frac{y_{1}^{3} y_{2}^{3} t}{\left(y_{1}-y_{2}\right)\left(y_{1}^{2}-y_{2}^{2} B_{1}^{2}\right)}=0$, and the result follows with $y_{1}^{2}-y_{2}^{2} B_{1}^{2}=y_{1}^{2}-y_{2}^{2}+4 y_{1}^{2} y_{2}^{2} t$.

For the degeneration side, we have the following result.

Theorem 8.2. $\Delta_{3} \Delta_{2} \Phi_{2}\left(y_{1}, y_{2}, t\right)=\operatorname{Esym}_{1,1}\left(\Delta_{3} \frac{y_{1}^{4} y_{2}}{y_{1}-y_{2}} t B_{1}^{-1}+3 y_{1}^{5} y_{2} t B_{1}^{-5}\right)$.

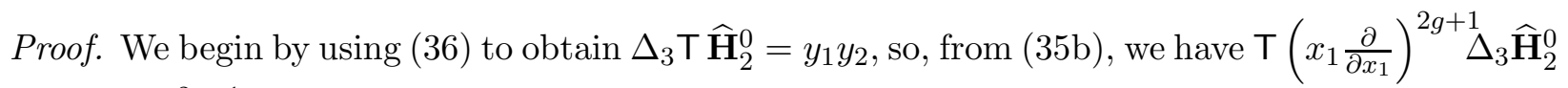
$=\left(y_{1}^{3} \frac{\partial}{\partial y_{1}}\right)^{2 g+1} y_{1} y_{2}=(4 g+1) ! ! y_{1}^{4 g+3} y_{2}$, where the second equality follows by induction. This gives

$$
\begin{aligned}
\Delta_{3} \Upsilon_{2} & =\operatorname{sym}_{1,1} \sum_{g \geq 1} 2^{2 g-1} \frac{(4 g+1) ! !}{(2 g-1) !} y_{1}^{4 g+3} y_{2} t^{2 g}=\operatorname{sym}_{1,1} y_{1}^{5} y_{2} t \sum_{g \geq 1} 3\left(\begin{array}{c}
-\frac{5}{2} \\
2 g-1
\end{array}\right)\left(-4 y_{1}^{2} t\right)^{2 g-1} \\
& =3 \mathrm{E} \mathrm{sym}_{1,1} y_{1}^{5} y_{2} t B_{1}^{-5} .
\end{aligned}
$$

Now, consider Lemma 7.5 with $m=2$, and apply $\Delta_{3}$ to both sides, to obtain $\Delta_{3} \Delta_{2} \Phi_{2}=$ $\Delta_{3} \operatorname{sym}_{1,1} \frac{y_{1}^{5} y_{2}}{y_{1}-y_{2}} \frac{\partial}{\partial y_{1}} \Phi_{1}\left(y_{1}, t\right)+\Delta_{3} \Upsilon_{2}$. The result follows from Corollary 7.4 and the expression for $\Delta_{3} \Upsilon_{2}$ above, since $\Delta_{3}$ and sym ${ }_{1,1}$ commute. 
Now we compare the results from the localization and degeneration sides, to obtain a proof of Faber's Conjecture for two parts.

Theorem 8.3. Faber's Intersection Number Conjecture is true for $n=2$.

Proof. By applying $\Delta_{3} \Delta_{2}$ to Theorem 8.1 , and from Theorem 8.2 , we routinely simplify to prove that $\Delta_{3} \Delta_{2} \Psi_{2}\left(y_{1}, y_{2}, t\right)$ and $\Delta_{3} \Delta_{2} \Phi_{2}\left(y_{1}, y_{2}, t\right)$ (under the condition that the Faber Conjecture is true for $n=2)$ are both equal to $\mathrm{Esym}_{1,1} \frac{2 y_{1}^{5} y_{2} t B_{1}^{-5}}{y_{1}-y_{2}}\left(3 y_{1}-2 y_{2}-10 y_{1}^{3} t+4 y_{1}^{2} y_{2} t+16 y_{1}^{5} t^{2}-8 y_{1}^{4} y_{2} t^{2}\right)$. Thus we have proved that if the Faber Conjecture is true for $n=2$, then $\Delta_{3} \Delta_{2} \Psi_{2}\left(y_{1}, y_{2}, t\right)=$ $\Delta_{3} \Delta_{2} \Phi_{2}\left(y_{1}, y_{2}, t\right)$. Then Proposition 7.6 implies that $\Psi_{2}\left(y_{1}, y_{2}, t\right)=\Phi_{2}\left(y_{1}, y_{2}, t\right)$, and the result follows from Lemma 7.2 .

8.2. Faber's Intersection Number Conjecture for three parts. The proof for three parts follows the steps and strategy of the proof for two parts, with no further technical difficulties. However, the full details of some of the expressions are somewhat longer, and we omit many below.

For the localization side, we determine that, if Faber's Conjecture is true for up to 3 parts, then

$$
\begin{aligned}
\Psi_{3}\left(y_{1}, y_{2}, y_{3}, t\right)= & \frac{1}{2} \operatorname{Esym}_{1,2} t \frac{d}{d t} t\left(1+B_{1}^{-1}\right) \frac{\widehat{Y}_{1}^{8} \widehat{Y}_{2} \widehat{Y}_{3}}{\left(\widehat{Y}_{1}^{2}-\widehat{Y}_{2}^{2}\right)\left(\widehat{Y}_{1}^{2}-\widehat{Y}_{3}^{2}\right)} \\
& -\frac{1}{2} \operatorname{Esym}_{1,1,1} t^{-2} \frac{d}{d t} t^{4}\left(1+B_{1}^{-1}\right) \frac{y_{1}^{2} y_{2}}{y_{1}-y_{2}} A_{1} \frac{\widehat{Y}_{1}^{6} \widehat{Y}_{3}}{\widehat{Y}_{1}^{2}-\widehat{Y}_{3}^{2}} \\
& +\frac{1}{2} \operatorname{Esym}_{1,2} t^{-2} \frac{d}{d t} t^{3}\left(1+B_{1}^{-1}\right) \frac{y_{1}^{6} y_{2} y_{3}}{\left(y_{2}-y_{1}\right)\left(y_{3}-y_{1}\right)} \widehat{Y}_{1}^{4} \\
& +\frac{1}{2} \operatorname{Esym}_{1,1,1} t\left(1+B_{1}^{-1}\right) \alpha+\frac{1}{2} \operatorname{Esym}_{1,2} t\left(1+B_{1}^{-1}\right) \beta,
\end{aligned}
$$

where

$$
\begin{aligned}
\alpha= & -\frac{y_{3}^{2} y_{2}}{y_{3}-y_{2}} A_{1}\left(\frac{\widehat{Y}_{1}^{4} \widehat{Y}_{3}^{3}}{\widehat{Y}_{1}^{2}-\widehat{Y}_{3}^{2}}+2 \frac{\widehat{Y}_{1}^{4} \widehat{Y}_{3}^{5}}{\left(\widehat{Y}_{1}^{2}-\widehat{Y}_{3}^{2}\right)^{2}}\right)+2 \frac{y_{1}^{2} y_{2}}{y_{1}-y_{2}} A_{1} \frac{\widehat{Y}_{1}^{8} \widehat{Y}_{3}}{\left(\widehat{Y}_{1}^{2}-\widehat{Y}_{3}^{2}\right)^{2}} \\
& +\frac{y_{1}^{3} y_{2}^{4} y_{3}}{\left(y_{2}-y_{3}\right)\left(y_{1}-y_{2}\right)^{2}} A_{1}^{2} \widehat{Y}_{1}^{2} \widehat{Y}_{2}+\frac{y_{1}^{6} y_{2} y_{3}}{\left(y_{2}-y_{1}\right)^{2}\left(y_{3}-y_{1}\right)} A_{1}^{2} \widehat{Y}_{1}^{3}, \\
\beta= & \left(1-y_{1}\right) \widehat{Y}_{1}^{4} \widehat{Y}_{2} \widehat{Y}_{3}+3 \frac{y_{1}^{5} y_{2} y_{3}}{\left(y_{2}-y_{1}\right)\left(y_{3}-y_{1}\right)} A_{1}^{2} \widehat{Y}_{1}^{3} .
\end{aligned}
$$

This is a large expression for $\Psi_{3}$, but the method of proof follows exactly that of Theorem 8.1, and is routine: we apply Corollary 5.1(b) with $m=3$, and parts (a)-(c) of Theorem 5.4, to obtain an expression for $\Lambda \mathbb{T}_{3}$, and use a smoothing of Faber's Conjecture for three parts to account for parts that are zero. The above expression then follows from (39).

For the degeneration side, we determine that

$$
\begin{aligned}
& \Delta_{2} \Phi_{3}\left(y_{1}, y_{2}, y_{3}, t\right)=\mathrm{E} \mathrm{sym}_{1,2} y_{1}^{5} y_{2} y_{3} t B_{1}^{-1}+3 \mathrm{E} \mathrm{sym}_{1,2} y_{1}^{5} y_{2} y_{3} t B_{1}^{-5} \\
& +\frac{1}{2} \operatorname{Esym}_{1,1,1}\left(\frac{y_{1}^{5} y_{3}}{y_{1}-y_{3}} \frac{\partial}{\partial y_{1}}+\frac{y_{2}^{5} y_{3}}{y_{2}-y_{3}} \frac{\partial}{\partial y_{2}}\right) t\left(1+B_{1}^{-1}\right)\left(\frac{\widehat{Y}_{1}^{4} \widehat{Y}_{2}}{\widehat{Y}_{1}^{2}-\widehat{Y}_{2}^{2}}-\frac{y_{1}^{2} y_{2}}{y_{1}-y_{2}} A_{1} \widehat{Y}_{1}^{2}\right) .
\end{aligned}
$$

The method of proof of this result follows exactly that of Theorem 8.2 we apply Lemma 7.5 with $m=3$, using the expression in Theorem 8.1 for $\Phi_{2}=\Psi_{2}$ and the fact that $\mathbf{T} \widehat{\mathbf{H}}_{3}=y_{1} y_{2} y_{3}$, from (36), which implies that $\Upsilon_{3}\left(y_{1}, y_{2}, y_{3}, t\right)=3 \mathrm{E} \mathrm{sym}_{1,2} y_{1}^{5} y_{2} y_{3} t B_{1}^{-5}$, giving the result.

Now we compare the results from the localization and degeneration sides, to obtain a proof of Faber's Conjecture for three parts.

Theorem 8.4. Faber's Intersection Number Conjecture is true for $n=3$. 
Proof. Applying $\Delta_{2}$ to (49), and from (50), with routine reductions (using Maple), we prove that $\Delta_{2} \Psi_{3}\left(y_{1}, y_{2}, y_{3}, t\right)=\Delta_{2} \Phi_{3}\left(y_{1}, y_{2}, y_{3}, t\right)$, under the condition that the Faber Conjecture is true for $n \leq 3$. Then Proposition 7.6 implies that $\Psi_{3}\left(y_{1}, y_{2}, y_{3}, t\right)=\Phi_{3}\left(y_{1}, y_{2}, y_{3}, t\right)$, and the result follows from Lemma 7.2 and Theorem 8.3 .

8.3. Intersection numbers with four or more parts. For the case of $n$ parts, on the localization side, we need to determine $\Lambda \xi_{n}^{(i)}$, for which we need an expression for the double Hurwitz series $\mathbf{H}_{n}^{0}$. Explicit expressions for the latter are known for $n=4$ [GJV2, Cor. 5.7] and $n=5$ [GJV2, Cor. 5.8], and with the help of Maple, we could in principle thus obtain expressions for $\Psi_{4}$ and $\Psi_{5}$ analogous to (49).

For the degeneration side, we apply Lemma 7.5, and note that $\Phi_{n}$ is obtained recursively from $\Phi_{j}, j<n$, once we have $\widehat{\mathbf{H}}_{n}^{0}$, which is given explicitly for all $n$ in (36). Thus we can obtain a rational expression for $\Phi_{n}$ for any $n$, by using Maple to help with the size of the expressions.

Together, via Lemma 7.2, these would enable us to prove Faber's Conjecture for four and five parts. However, we are prevented from proving the result for larger values of $n$ because we do not have explicit expressions for the double Hurwitz series $\mathbf{H}_{n}^{0}$, and we do not see how to obtain an explicit formula that holds for all $n$. In order to use our methodology to prove the result for arbitrary $n$, we would need to use our functional equations for the various tree series given in Theorem 4.4, and then use the Join-cut Equation for the double Hurwitz series.

\section{Appendix A. Proofs of Theorems 5.3 and 5.4}

In this Appendix we develop the substantial amount of material that is required to determine $\Lambda \xi_{m}^{(i)}$ for $m=1,2,3$, and is not required elsewhere in the paper. A key ingredient is the symmetrization of the double Hurwitz generating series.

A.1. Preliminaries. (a) Completing the symmetrization on the localization side: In order to determine $\Lambda \xi_{m}^{(i)}$ we have the following result, which together with Corollary [5.1 completes the symmetrization of Theorem 4.4 (the third form of the Localization Tree Theorem). It requires the symmetrized series

$$
\begin{cases}f_{j, m}\left(x_{1}, \ldots, x_{m}, u\right) & \left.\left.:=\Xi_{m} f_{j}(z, u ; \mathbf{p})\right), \quad g_{j, m}\left(x_{1}, \ldots, x_{m}, u\right):=\Xi_{m} g_{j}(z, u ; \mathbf{p})\right), \\ \mathbf{H}_{m}^{0}\left(x_{1}, \ldots, x_{m}, u ; \mathbf{q}\right) & :=\Xi_{m} H^{0}(z, u ; \mathbf{p}, \mathbf{q}),\end{cases}
$$

for $m \geq 1$, and the substitution operator

$$
\Omega: q_{i} \mapsto \frac{i^{i-1}}{i !}, \quad i \geq 1 .
$$

Corollary A.1. For $m \geq 1$,

$$
\text { a) } \xi_{m}^{(i)}=\sum_{j \geq 1} \frac{j^{j+i}}{j !} f_{j, m}, \quad i \geq 0
$$

where, for $j \geq 1$,

$$
\begin{aligned}
& \text { b) } f_{j, m}=\sum_{\substack{k \geq 0, j_{1}, \ldots, j_{k} \geq 1, i_{0}, \ldots, i_{k} \geq 1, i_{0}+\cdots+i_{k}=m}} \operatorname{sym}_{i_{0}, \ldots, i_{k}} \frac{u^{-2}}{k !}\left(\Omega j \frac{\partial^{k+1}}{\partial q_{j} \partial q_{j_{1}} \cdots \partial q_{j_{k}}} \mathbf{H}_{i_{0}}^{0}\left(x_{\rho_{0}}, u ; \mathbf{q}\right)\right) \prod_{a=1}^{k} g_{j_{a}, i_{a}}\left(x_{\rho_{a}}, u\right), \\
& \text { c) } g_{j, m}=\sum_{\substack{k \geq 0, j_{1}, \ldots, j_{k} \geq 1, i_{1}, \ldots, i_{k} \geq 1, i_{1}+\cdots+i_{k}=m}} \operatorname{sym}_{i_{1}, \ldots, i_{k}} \frac{\left(j+j_{1}+\cdots+j_{k}\right)^{k-2}}{k !} \frac{j^{j+1}}{j !} \prod_{a=1}^{k} \frac{j_{a}^{j_{a}}}{j_{a} !} f_{j_{a}, i_{a}}\left(x_{\rho_{a}}, u\right) .
\end{aligned}
$$


Proof. The result follows by applying $\Xi_{m}$ to (28) Theorem 4.4(c) and (d), for $m \geq 1$, and using the properties of $\Xi_{m}$ given in Lemmas 4.1-4.3 of [GJVn.

We shall determine $\Lambda \xi_{m}^{(i)}, m=1,2,3$, by applying Corollary A.1 directly. The results that are needed for the series $\mathbf{H}_{m}^{0}$ and its partial derivatives are given in the next two sections.

(b) The symmetrized double Hurwitz series, genus 0: Now let $Q(t):=\sum_{j \geq 1} q_{j} t^{j}$, and $v$ be the unique formal power series solution of the functional equation

$$
v=x e^{u Q(v)} .
$$

Let

$$
v_{i}:=v\left(x_{i}\right), \quad Q_{i}:=Q\left(v_{i}\right), \quad \mu(t):=\left(1-u t Q^{\prime}(t)\right)^{-1}, \quad \mu_{i}:=\mu\left(v_{i}\right) .
$$

Then in GJV2], we proved that

$$
x_{1} \frac{\partial}{\partial x_{1}} \mathbf{H}_{1}^{0}=u Q_{1}, \quad \mathbf{H}_{2}^{0}=\log \left(\frac{v_{1}-v_{2}}{x_{1}-x_{2}}\right)-u Q_{1}-u Q_{2}, \quad \mathbf{H}_{3}^{0}=\sum_{i=1}^{3}\left(\mu_{i}-1\right) \prod_{\substack{1 \leq j \leq 3, j \neq i}} \frac{v_{j}}{v_{i}-v_{j}} .
$$

(c) Partial derivatives of the symmetrized double Hurwitz series: Differentiating (53) gives

$$
x_{1} \frac{\partial}{\partial x_{1}} v_{1}=v_{1} \mu_{1}, \quad \frac{\partial}{\partial q_{j}} v_{1}=u v_{1}^{j+1} \mu_{1},
$$

and from (55) and (56) we obtain

$$
\frac{\partial}{\partial q_{j}} \mathbf{H}_{2}^{0}=\frac{u v_{1}^{j+1} \mu_{1}-u v_{2}^{j+1} \mu_{2}}{v_{1}-v_{2}}-u v_{1}^{j} \mu_{1}-u v_{2}^{j} \mu_{2}=\operatorname{sym}_{1,1} \frac{u v_{1}^{j} \mu_{1} v_{2}}{v_{1}-v_{2}} .
$$

For partial derivatives of $\mathbf{H}_{1}^{0}$ in general, we have the following result.

Lemma A.2. (a) For $j \geq 1, \frac{\partial}{\partial q_{j}} \mathbf{H}_{1}^{0}=\frac{u}{j} v_{1}^{j}$.

(b) For $k \geq 1, j_{1}, \ldots, j_{k} \geq 1, \frac{\partial^{k}}{\partial q_{j_{k}} \cdots \partial q_{j_{1}}} \mathbf{H}_{1}^{0}=u^{k}\left(\mu_{1} v_{1} \frac{\partial}{\partial v_{1}}\right)^{k-2}\left(\mu_{1} v_{1}^{j_{1}+\cdots+j_{k}}\right)$.

Proof. Differentiating (55), we obtain

$$
\frac{\partial}{\partial q_{j}} x_{1} \frac{\partial}{\partial x_{1}} \mathbf{H}_{1}^{0}=u Q^{\prime}\left(v_{1}\right) \frac{\partial v_{1}}{\partial q_{j}}+u v_{1}^{j}=u Q^{\prime}\left(v_{1}\right) u v_{1}^{j+1} \mu_{1}+u v_{1}^{j}=u v_{1}^{j} \mu_{1},
$$

where we have used (56) for the second equality. Dividing by $x_{1}$ and integrating, we obtain $\frac{\partial}{\partial q_{j}} \mathbf{H}_{1}^{0}=$ $\int_{0}^{x_{1}} u v_{1}^{j} \mu_{1} \frac{d x_{1}}{x_{1}}=\int_{0}^{v_{1}} u v_{1}^{j-1} d v_{1}$, where we have used (56) to change variables for the second equality. Part (a) follows immediately.

We prove part (b) by induction on $k$. For the base case, note that the result is true for $k=1$, since this is equivalent to part (a) above. Now assume that the result holds for $k-1 \geq 1$, and note that (56) implies the operator identity $\mu_{1} v_{1} \partial / \partial v_{1}=x_{1} \partial / \partial x_{1}$, so we have

$$
\begin{aligned}
\frac{\partial^{k}}{\partial q_{j_{k}} \cdots \partial q_{j_{1}}} \mathbf{H}_{1}^{0} & =\frac{\partial}{\partial q_{j_{k}}} u^{k-1}\left(x_{1} \frac{\partial}{\partial x_{1}}\right)^{k-2}\left(\mu_{1} v_{1} \frac{\partial}{\partial v_{1}}\right)^{-1}\left(\mu_{1} v_{1}^{j_{1}+\cdots+j_{k-1}}\right) \\
& =u^{k-1}\left(x_{1} \frac{\partial}{\partial x_{1}}\right)^{k-2} \frac{\partial}{\partial q_{j_{k}}}\left(\frac{v_{1}^{j_{1}+\cdots+j_{k-1}}}{j_{1}+\cdots+j_{k-1}}\right)
\end{aligned}
$$

and part (b) follows from (56) and the operator identity. 
(d) Polynomiality and the symmetrized double Hurwitz series: From Corollary A.1(a), (b), to evaluate $\Lambda \xi_{m}^{(i)}$ we need to apply the substitution $\Lambda \Omega$ to partial derivatives of the symmetrized double Hurwitz series $\mathbf{H}_{m}^{0}$, which we have shown above can be expressed in terms of $v_{i}$ and $\mu_{i}$. Thus define

$$
V_{i}:=\Lambda \Omega v_{i} .
$$

In the following result, the action of the change of variables $C$, the second step in our fundamental transformation, is given for expressions in $V_{i}$. Note how indirectly we proceed in the proof, especially for part (a), as referred to in the discussion following (33). This result is the key to polynomiality on the localization side.

Lemma A.3. For $i \geq 1$,
a) C $y\left(V_{i}\right)=\frac{(1-u) y_{i}}{1-u y_{i}}$
b) $\mathrm{C} \Lambda \Omega \mu_{i}=\frac{1-u y_{i}}{1-u}$
c)
$\mathrm{C} V_{i} \frac{\partial}{\partial V_{i}}=y_{i}^{2}\left(y_{i}-1\right) \frac{1-u}{1-u y_{i}} \frac{\partial}{\partial y_{i}} \mathrm{C}$.

Proof. For part (a), the series $w=w(x)$ defined in (33) $)$ is the unique formal power series solution to $w=x e^{w}$, which we shall call the tree equation (see, e.g., [GJ1, Ex. 1.2.5]). Now $V_{i}=V\left(x_{i}\right)$, where, from (53), $V=V(x)$ is the unique formal power series solution to

$$
V=(1-u)^{-1} x \exp \left(-u \sum_{j \geq 1} \frac{j^{j-1}}{j !} V^{j}\right)=(1-u)^{-1} x e^{-u w(V)},
$$

where the second equality follows from (133). But the tree equation gives $w(V)=V e^{w(V)}$, and eliminating $V$ between this equation and (59), we obtain $(1-u) w(V)=x e^{(1-u) w(V)}$. Comparing this to the tree equation, we conclude that $(1-u) w(V(x))=w(x)$, and by $(33 \mathrm{~b})$, this implies

$$
y\left(V_{i}\right)=\frac{(1-u) y_{i}}{1-u y_{i}}
$$

where here $y_{i}=y\left(x_{i}\right)$ is a series in $x_{i}$. Part (a) follows immediately.

For part (b), from (54) and (33b) we have

$$
\Lambda \Omega \mu_{i}=\frac{1}{1+u \sum_{j \geq 1} \frac{j^{j}}{j !} V_{i}^{j}}=\frac{1}{1+u\left(y\left(V_{i}\right)-1\right)},
$$

and part (b) follows immediately from part (a).

For part (c), from (35a), we have

$$
V_{i} \frac{\partial}{\partial V_{i}}=y\left(V_{i}\right)^{2}\left(y\left(V_{i}\right)-1\right) \frac{\partial}{\partial y\left(V_{i}\right)} .
$$

But, by differentiating (60), we obtain

$$
\frac{\partial y\left(V_{i}\right)}{\partial y_{i}}=\frac{1-u}{\left(1-u y_{i}\right)^{2}}=\frac{y\left(V_{i}\right)^{2}}{(1-u) y_{i}^{2}}
$$

which implies C $y\left(V_{i}\right)^{2} \frac{\partial}{\partial y\left(V_{i}\right)}=(1-u) y_{i}^{2} \frac{\partial}{\partial y_{i}} \mathrm{C}$, and part (c) follows immediately from (61).

(e) Two results: In the proofs of Section A.2, we make use of the identity

$$
\sum_{j, k \geq 1} \frac{1}{j+k} \frac{j^{j+1}}{j !} V_{1}^{j} \frac{k^{k}}{k !} V_{2}^{k}=\frac{V_{2}}{V_{2}-V_{1}}-y\left(V_{1}\right)^{2} \frac{y\left(V_{2}\right)-1}{y\left(V_{2}\right)-y\left(V_{1}\right)},
$$

whose proof follows from [GJ2, and the fact (an easy induction) that

$$
\left(\frac{y_{i}^{3}}{1-u y_{i}} \frac{\partial}{\partial y_{i}}\right)^{i} \frac{y_{i}}{1-u y_{i}}=(2 i-1) ! !\left(\frac{y_{i}}{1-u y_{i}}\right)^{2 i+1}, \quad i \geq 1 .
$$


A.2. The proofs. Proofs of Theorem 5.3(a) $(m=1)$, and Theorem 5.4(a): From Corollary A.1(b) with $m=1$, Lemma $\mathbf{A . 2}$ (a) and (58), we have

$$
\Lambda f_{j, 1}=u^{-2} \Lambda \Omega j \frac{\partial}{\partial q_{j}} \mathbf{H}_{1}^{0}=-u^{-1} \Lambda \Omega v_{1}^{j}=-u^{-1} V_{1}^{j}
$$

Then from Corollary A.1(a) with $m=1$, we obtain

$$
\Lambda \xi_{1}^{(i)}=\sum_{j \geq 1} \frac{j^{j+i}}{j !} \Lambda f_{j, 1}=-u^{-1} \sum_{j \geq 1} \frac{j^{j+i}}{j !} V_{1}^{j}=-u^{-1}\left(V_{1} \frac{\partial}{\partial V_{1}}\right)^{i}\left(y\left(V_{1}\right)-1\right),
$$

from (33b). But Lemma A.3(a) and (c) give

$$
\mathrm{C} \Lambda \xi_{1}^{(i)}=-u^{-1}(1-u)^{i}\left(y_{1}^{2} \frac{y_{1}-1}{1-u y_{1}} \frac{\partial}{\partial y_{1}}\right)^{i}\left(\frac{(1-u) y_{1}}{1-u y_{1}}-1\right)
$$

and Theorem 5.3(a) $(m=1)$ follows immediately. Then $\mathrm{T} \Lambda \xi_{1}^{(i)}=\mathrm{T} u^{-1}(1-u)^{i+1}\left(\frac{y_{1}^{3}}{1-u y_{1}} \frac{\partial}{\partial y_{1}}\right)^{i}$ $\frac{y_{1}}{1-u y_{1}}$, and Theorem 5.4(a) follows immediately from (63).

Proofs of Theorem 5.3(a) $(m=2)$, and Theorem 5.4(b): From Corollary A.1(c) with $m=1$, and (64), we obtain

$$
\Lambda g_{j_{1}, 1}\left(x_{1}, u\right)=\sum_{j_{2} \geq 1} \frac{1}{j_{1}+j_{2}} \frac{j_{1}^{j_{1}+1}}{j_{1} !} \frac{j_{2}^{j_{2}}}{j_{2} !} \Lambda f_{j_{2}, 1}\left(x_{1}, u\right)=-u^{-1} \frac{j_{1}^{j_{1}+1}}{j_{1} !} \sum_{j_{2} \geq 1} \frac{1}{j_{1}+j_{2}} \frac{j_{2}^{j_{2}}}{j_{2} !} V_{1}^{j_{2}},
$$

and from Corollary A.1(b) with $m=2$, we obtain $\Lambda f_{j, 2}=S_{1}+S_{2}$, where

$$
S_{1}=u^{-2} \Lambda \Omega j \frac{\partial}{\partial q_{j}} \mathbf{H}_{2}^{0}, \quad S_{2}=u^{-2} \operatorname{sym}_{1,1} \sum_{j_{1} \geq 1} \Lambda g_{j_{1}, 1}\left(x_{2}, u\right) \Omega j \frac{\partial^{2}}{\partial q_{j} \partial q_{j_{1}}} \mathbf{H}_{1}^{0}\left(x_{1}, u\right) .
$$

We can determine $S_{1}$ immediately from (157), giving $S_{1}=-u^{-1} j \operatorname{sym}_{1,1} \frac{V_{1}^{j} V_{2}}{V_{1}-V_{2}} \Lambda \Omega \mu_{1}$. To determine $S_{2}$, use Lemma A.2 with $k=2$ and (65), to obtain $S_{2}=-u^{-1} j \operatorname{sym}_{1,1} V_{1}^{j}\left(\Lambda \Omega \mu_{1}\right) \sum_{j_{1}, j_{2} \geq 1} \frac{1}{j_{1}+j_{2}} \frac{j_{1}^{j_{1}+1}}{j_{1} !} V_{1}^{j_{1}}$

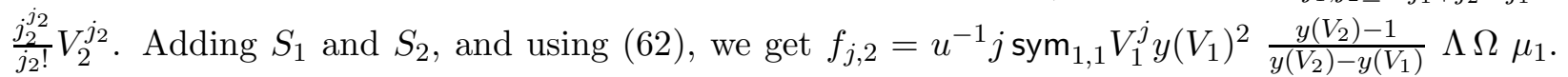
Then from Corollary A.1(a) with $m=2$, we obtain

$$
\begin{aligned}
\Lambda \xi_{2}^{(i)} & =\sum_{j \geq 1} \frac{j^{j+i}}{j !} \Lambda f_{j, 2}=u^{-1} \operatorname{sym}_{1,1} y\left(V_{1}\right)^{2} \frac{y\left(V_{2}\right)-1}{y\left(V_{2}\right)-y\left(V_{1}\right)}\left(\Lambda \Omega \mu_{1}\right) \sum_{j \geq 1} \frac{j^{j+i+1}}{j !} V_{1}^{j} \\
& =u^{-1} \operatorname{sym}_{1,1} y\left(V_{1}\right)^{2} \frac{y\left(V_{2}\right)-1}{y\left(V_{2}\right)-y\left(V_{1}\right)}\left(\Lambda \Omega \mu_{1}\right)\left(V_{1} \frac{\partial}{\partial V_{1}}\right)^{i+1}\left(y\left(V_{1}\right)-1\right),
\end{aligned}
$$

from (33b). Now Lemma A.3(a),(b),(c) give

$$
\mathrm{C} \Lambda \xi_{2}^{(i)}=u^{-1}(1-u)^{i+1} \operatorname{sym}_{1,1} \frac{y_{1}^{2}\left(y_{2}-1\right)}{y_{2}-y_{1}}\left(y_{1}^{2} \frac{y_{1}-1}{1-u y_{1}} \frac{\partial}{\partial y_{1}}\right)^{i+1}\left(\frac{(1-u) y_{1}}{1-u y_{1}}-1\right)
$$

and Theorem $5.3(\mathrm{a})(m=2)$ follows immediately. Then we have

$$
\mathrm{T} \Lambda \xi_{2}^{(i)}=\mathrm{T} u^{-1}(1-u)^{i+2} \operatorname{sym}_{1,1} \frac{y_{1}^{2} y_{2}}{y_{2}-y_{1}}\left(\frac{y_{1}^{3}}{1-u y_{1}} \frac{\partial}{\partial y_{1}}\right)^{i+1} \frac{y_{1}}{1-u y_{1}},
$$

and Theorem 5.4(b) follows immediately from (63).

Outline of proofs of Theorem 5.3(a) $(m=3)$, and Theorem 5.4(c): The method of proof of these results follows those for the cases $m=1,2$ above, with no further technical difficulties. We give only an outline. We first determine $\Lambda f_{j, 3}$ from Corollary A.1(b) This requires $\Lambda g_{j, 2}$, which we 
determine from Corollary A.1(c), and the partial derivatives $\frac{\partial^{3}}{\partial q_{j} \partial q_{j_{1}} \partial q_{j_{2}}} \mathbf{H}_{1}^{0}, \frac{\partial^{2}}{\partial q_{j} \partial q_{j_{1}}} \mathbf{H}_{2}^{0}$ and $\frac{\partial}{\partial q_{j}} \mathbf{H}_{3}^{0}$. But rational expressions for these partials are routinely obtained, from Lemma A.2(b) for $\mathbf{H}_{1}^{0}$, and by applying the chain rule and (56) to (57) and (55). We then use Corollary A.1(a) with $m=3$ to obtain an explicit expression for $\Lambda \xi_{3}^{(i)}$, and then apply $\mathrm{C}$ to prove Theorem $5.3(\mathrm{a})(m=3)$. Then Theorem 5.4(c) follows immediately. 


\section{Appendix B. Glossary of Notation}

If a symbol in column 1 has an argument, it appears as the first item of the entry in column 3. In column 2, "C" denotes "Corollary", "Cn" denotes "Conjecture", "D" denotes "Definition", "P" denotes "Proposition" "T" denotes "Theorem", "b" denotes "immediately before", "f" denotes "immediately following" and "I" denotes "Introduction to".

\begin{tabular}{|c|c|c|c|c|c|}
\hline & $\mathrm{f}(21)$ & $\begin{array}{l}\text { Removal of contribution of } \\
\text { root from corresponding prod- } \\
\text { uct } \\
\text { Root-vertex of tree } t\end{array}$ & $\begin{array}{l}\rho_{j} \\
\Psi_{m}\end{array}$ & $\frac{(30)}{(39)}$ & $\begin{array}{l}\text { Used in } \Xi_{m} \\
\left(y_{1}, \ldots, y_{m}, t\right): \text { Generating se- } \\
\text { ries for } \mathrm{T} \Lambda \mathbb{T}_{m}^{g} / \mathbb{G}_{g, 1} \\
\text { First Chern class }\end{array}$ \\
\hline & $\mathrm{D} 4.2$ & Combinatorial $\mathrm{p}$ & $\Omega$ & 52 & Substitution operator $q_{i} \mapsto$ \\
\hline$k] f$ & - & $\begin{array}{l}\text { Coefficient of } x^{k} \text { in a formal } \\
\text { power series } f\end{array}$ & $\Omega^{\mathrm{br}}, \Omega_{0}^{\mathrm{br}}$ & 94.2 & $\begin{array}{l}\frac{i^{i-1}}{i !} \\
\text { Variants of branch decomposi- }\end{array}$ \\
\hline$\cdot]_{\eta}$ & $\mathrm{D} 4.1$ & $\begin{array}{l}\mathcal{A} \text {, wt : Exponential generat- } \\
\text { ing series }\end{array}$ & $\begin{array}{l}\Omega_{\infty}^{\mathrm{br}} \\
A_{i}\end{array}$ & (3)) & 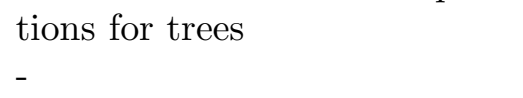 \\
\hline & Ig3 & Sum of parts of $\alpha$ & $A(\mathfrak{t})$ & 11) & function for $\mathfrak{t}$ \\
\hline$\rangle_{g}^{\mathbb{F a b}}$ & (3) & $\begin{array}{l}\tau_{a_{1}} \cdots \tau_{a_{n}} \lambda_{k} \quad: \text { Faber } \\
\text { for } \pi_{*}\left(\psi_{1}^{a_{1}} \cdots \psi_{n}^{a_{n}} \lambda_{k}\right)\end{array}$ & $\begin{array}{l}B(\mathfrak{t}) \\
B_{i}\end{array}$ & $(20)$ & unction for $t$ \\
\hline$\cdot\rangle_{g}^{\mathrm{Fab}}$ & (41) & $\tau_{a_{1}} \cdots \tau_{a_{n}} \lambda_{k}: \mathrm{F}$ & $b r$ & ( & ade \\
\hline & I 83 & $d$ : branching over $\infty$ & $C(\mathfrak{t}$ & (1) & \\
\hline & (18) & $\coprod_{v \in}$ & & $\mathrm{f}(\overrightarrow{33})$ & $\mathrm{Ch}$ \\
\hline & 33.1. & Ptn & $D(\mathfrak{t})$ & 20 & \\
\hline & $\mathrm{D} 3.1$ & edges & & $\mathrm{b}$ (43) & Ev \\
\hline & $\mathrm{D} 3.10$ & edges & $\begin{array}{l}\mathcal{E}_{0 \infty}(\mathfrak{t}) \\
\mathcal{E}_{\infty t}(\mathfrak{t})\end{array}$ & 0 & $\begin{array}{l}\mathrm{Se} \\
\mathrm{Se}\end{array}$ \\
\hline & (44) & Part & & & \\
\hline & $\mathrm{D} 3.10$ & -edges & 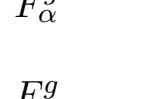 & & note $\mathbb{F}^{g, \alpha}=F_{\alpha}^{g} \mathbb{G}_{g, 1}$ \\
\hline e) & $\mathrm{D}$ & Wei & $F$ & & $\begin{array}{l}(z ; \mathbf{p}) \text { : Generating series for } \\
\text { Faber numbers } F_{\alpha}^{g}\end{array}$ \\
\hline$(\mathfrak{t})$ & $\begin{array}{l}\mathrm{D} 3 . \\
\mathrm{bD}[\end{array}$ & $\begin{array}{l}\text { tices in } \mathfrak{t} \\
\text { incident }\end{array}$ & $F_{m}^{g}$ & 11) & $\begin{array}{l}\left(x_{1}, \ldots, x_{m}\right) \quad \text { Symmetrized } \\
F^{g}(z ; \mathbf{p})\end{array}$ \\
\hline$m$ & (45) & $\left(y_{1}\right.$ & & & Generating series $f$ \\
\hline & $\$ 2.2$ & $" \kappa-c$ & & & $\begin{array}{l}\left(x_{1}, \ldots, x_{m}, u\right): \\
\text { series for } \mathcal{T}_{0, j}\end{array}$ \\
\hline & (32) & $\begin{array}{l}\text { Substitution operator } u \mapsto \\
-u, x_{i} \mapsto(1-u)^{-1} x_{i} \\
\lambda \text {-class }\end{array}$ & $\mathbb{G}_{g, 1}$ & $\mathrm{~T} 3.5$ & $\begin{array}{l}\text { A "natural generator" of } \\
R_{2 g-1}\left(\mathcal{M}_{g, 1}\right), \text { C6.3 }\end{array}$ \\
\hline & $(54)$ & or double $\mathrm{H}_{\mathrm{l}}$ & $\mathbb{G}_{g}$ & & 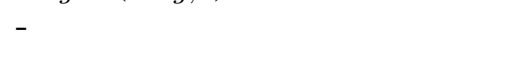 \\
\hline & (29) & trization & $G$ & & $\begin{array}{ll}- & - \\
\end{array}$ \\
\hline & (28) & $\begin{array}{l}(z, u ; \mathbf{p}) \text { : Localization tree se- } \\
\text { ries }\end{array}$ & & & ing series for \\
\hline & (31) & zed lo- & & & series for $\mathcal{T}_{\infty, j}$ \\
\hline & (40) & rating se- & $\begin{array}{l}H_{\alpha}^{0} \\
H_{\alpha,}^{0}\end{array}$ & & $\begin{array}{l}\text { Genus } 0 \text { (single) Hurwitz no. } \\
\text { Genus } 0 \text { double Hurwitz no. }\end{array}$ \\
\hline
\end{tabular}




\begin{tabular}{|c|c|c|c|c|c|}
\hline $\mathbb{H}_{j}^{g, \alpha}$ & 33.2 & Hurwitz class & $R^{*}\left(\mathcal{M}_{g, n}^{r t}\right)$ & I乌1 & Tautological ring \\
\hline$\widehat{H}^{0}$ & (14) & $\begin{array}{l}(z ; \mathbf{p}): \quad \text { Gen. } \\
\text { genus } 0 \text { series for } \\
\text { nos. } H_{\alpha}^{0}\end{array}$ & $r_{\alpha}^{g}$ & (4) & $\begin{array}{l}\mathcal{M}_{g, n}^{r t} \\
\text { "Expected" no. of } \\
\text { branch pts. away from }\end{array}$ \\
\hline$H^{0}$ & (27) & $\begin{array}{l}(z, u ; \mathbf{p} ; \mathbf{q}): \text { Gen. series } \\
\text { for genus } 0 \text { double Hur- } \\
\text { witz nos. } H_{\alpha, \beta}^{0}\end{array}$ & $r_{\alpha, \beta}^{g}$ & (6) & $\begin{array}{l}\infty \\
\text { "Expected" no. of } \\
\text { branch pts. away from } 0 \\
\text { and } \infty\end{array}$ \\
\hline$\widehat{\mathbf{H}}_{m}^{0}$ & (31) & $\begin{array}{l}\left(x_{1}, \ldots, x_{m}\right): \quad \text { Sym- } \\
\text { metrized single Hurwitz } \\
\text { series }\end{array}$ & $r_{g, \alpha}^{\mathrm{Fab}}$ & (9) & $\begin{array}{l}\text { Number of fixed branch } \\
\text { pts. on } \mathbb{P}^{1} \\
\text { - }\end{array}$ \\
\hline $\mathbf{H}_{m}^{0}$ & (51) & $\begin{array}{l}\left(x_{1}, \ldots, x_{m}, u ; \mathbf{q}\right): \text { Sym- } \\
\text { metrized double Hurwitz }\end{array}$ & $R_{j}(\mathcal{M})$ & S2.2 & $\begin{array}{l}R^{\operatorname{dim} \mathcal{M}-j}(\mathcal{M}) \text { for open } \\
\text { subset } \mathcal{M} \text { of } \overline{\mathcal{M}}_{g, n}\end{array}$ \\
\hline$L$ & $\mathrm{P} 3.1$ & $\begin{array}{l}\text { series, genus } 0 \\
\text { Class in } \operatorname{Sym}_{\alpha, \beta}^{r^{g}}\left(\mathbb{P}^{1}\right)\end{array}$ & $\mathrm{S}_{i}$ & fT5.3 & $\begin{array}{l}\text { Operator for terms of to- } \\
\text { tal degree } i\end{array}$ \\
\hline$l(\alpha)$ & & Number of parts of $\alpha$ & $\operatorname{sym}_{i_{1}, ., i_{k}}$ & $\mathrm{f}(30)$ & Summation operator for \\
\hline $\mathcal{M}_{g}$ & 92.1 & $\begin{array}{l}\text { Moduli space of smooth } \\
\text { curves }\end{array}$ & $\mathcal{T}_{g, m}, \mathcal{T}_{0, j}$ & $\mathrm{D} 3.10$ & $\begin{array}{l}\text { symmetrization } \\
\text { Sets of localization trees }\end{array}$ \\
\hline$\overline{\mathcal{M}}_{g, \alpha}\left(\mathbb{P}^{1}\right)$ & $\$ 2.1$ & $\begin{array}{l}\text { Moduli space of stable } b \text { - } \\
\text { pointed genus } g \text { curves } \\
\text { Moduli space of genus } g\end{array}$ & $\begin{array}{l}\mathcal{T}_{\infty, j} \\
\mathrm{~T}, \mathrm{~T}^{\prime}\end{array}$ & $\$ 5.3$ & $\begin{array}{l}\text { Operators for maximum } \\
\text { degree }\end{array}$ \\
\hline & & $\begin{array}{l}\text { relative stable maps to } \mathbb{P}^{1} \\
\text { relative to one } \mathrm{pt.} \infty\end{array}$ & $\mathrm{T} \Xi_{m}$ & $\$ 5$ & $\begin{array}{l}\text { Fundamental transfor- } \\
\text { mation }\end{array}$ \\
\hline$\overline{\mathcal{M}}_{g, \alpha, \beta}\left(\mathbb{P}^{1}\right)$ & 33.1 .2 & $\begin{array}{l}\text { Moduli space of relative } \\
\text { stable maps to } \mathbb{P}^{1} \text { relative } \\
\text { to two points } 0 \text { and } \infty\end{array}$ & $\mathbb{T}^{g}$ & $\mathrm{~T} 4.4(\mathrm{~b})$ & $\begin{array}{l}(z, u ; \mathbf{p}) \text { : Tree sum re- } \\
\text { lated to generating series } \\
\text { for } \mathbb{F}_{g, \alpha}\end{array}$ \\
\hline$\overline{\mathcal{M}}_{g, \alpha, \beta}\left(\mathbb{P}^{1}\right)_{\sim}$ & 3.1 .2 & $\begin{array}{l}\text { Moduli space of "rubber } \\
\text { maps" }\end{array}$ & $\mathbb{T}_{m}^{g}$ & (31) & $\begin{array}{l}\left(x_{1}, \ldots, x_{m}, u\right): \quad \text { Sym- } \\
\text { metrized } \mathbb{T}^{g}(z, u ; \mathbf{p})\end{array}$ \\
\hline $\mathcal{M}_{g, \alpha}\left(\mathbb{P}^{1}\right)^{r t}$ & 33.1 .3 & Moduli space of relative & $\mathfrak{t}$ & $\mathrm{D} 3.10$ & Localization tree \\
\hline & & stable maps with rational & $\mathcal{V}_{0}(\mathfrak{t})$ & $\mathrm{D} 3.10$ & Set of 0 -vertices in $\mathfrak{t}$ \\
\hline & & tails & $\mathcal{V}_{\infty}(\mathfrak{t})$ & $\mathrm{D} 3.10$ & Set of $\infty$-vertices in $\mathfrak{t}$ \\
\hline $\mathcal{M}_{g, \alpha, \beta}\left(\mathbb{P}^{1}\right)^{r t}$ & $\$ 3.1 .3$ & Similar to the above & $\mathcal{V}_{t}(\mathfrak{t})$ & $\mathrm{D} 3.10$ & Set of $t$-vertices in $\mathfrak{t}$ \\
\hline $\mathcal{M}_{g, \alpha}\left(\mathbb{P}^{1}\right)$ & $\$ 3.1 .4$ & Possibly disconnected & $\mathcal{U}_{k}(\mathcal{S})$ & fL4.3 & Unordered list \\
\hline $\mathbb{P}_{m}^{g}(\ldots)$ & (19) & $\begin{array}{l}\text { moduli space } \\
\left(\alpha_{1}, \ldots, \alpha_{m}\right): \quad \text { Faber } \\
\text { polynomial }\end{array}$ & $v, v_{i}$ & (53), (54) & $\begin{array}{l}\text { Implicitly defined indets. } \\
\text { for double Hurwitz series } \\
\text { 0-vertex labelled } i\end{array}$ \\
\hline $\mathcal{P}$ & I 93 & $\begin{array}{l}\text { Set of all non-empty par- } \\
\text { titions }\end{array}$ & $\begin{array}{l}V_{i} \\
w, w_{i}\end{array}$ & $(53 \mathrm{ag})$ & $\begin{array}{l}\text { Transform of } v_{i} \\
\text { Rooted tree series }\end{array}$ \\
\hline $\mathbf{p}$ & (27) & $\left(p_{1}, p_{2}, \ldots\right)$ & $\mathfrak{w}_{\gamma}$ & $\longdiv { 4 . 2 }$ & Star of $t$-vertices rooted \\
\hline$p_{i}$ & $\mathrm{f}(14)$ & $\begin{array}{l}\text { Indeterminate marking a } \\
\text { part } i \text { in } \alpha\end{array}$ & $x_{i}$ & (29) & $\begin{array}{l}\text { at an } \infty \text {-vertex } \\
\text { Indeterminates for sym- }\end{array}$ \\
\hline$Q(t)$ & (54) & $\begin{array}{l}\text { Generating series for in- } \\
\text { determinates } q_{i}\end{array}$ & & (48) & $\begin{array}{l}\text { metrized series } \\
-\end{array}$ \\
\hline q & (27) & $\left(q_{1}, q_{2}, \ldots\right)$ & $Y_{i}(u)$ & (34) & - \\
\hline$q_{i}$ & $\mathrm{f}(27)$ & $\begin{array}{l}\text { Indeterminate marking a } \\
\text { part } i \text { in } \beta\end{array}$ & $y, y_{i}$ & $(\overrightarrow{33} \mathrm{~b})$ & $\begin{array}{l}\text { Implicitly defined indets. } \\
\text { for symmetrized series }\end{array}$ \\
\hline
\end{tabular}

\section{REFERENCES}

[BCT] A. Bertram, R. Cavalieri, and G. Todorov, Evaluating tautological classes using only Hurwitz numbers, math.AG/0608656.

[BP] J. Bryan and R. Pandharipande, The local Gromov-Witten theory of curves, math.AG/0411037. 
[C] R. Cavalieri, Generating functions for Hurwitz-Hodge integrals, math.AG/0608590

[DM] C. Deninger and J. Murre, Motivic decomposition of abelian schemes and the Fourier transform, J. Reine Angew. Math. 422 (1991), 201-219.

[ELSV1] T. Ekedahl, S. Lando, M. Shapiro, and A. Vainshtein, On Hurwitz numbers and Hodge integrals, C. R. Acad. Sci. Paris Sér. I Math. 328 (1999), 1175-1180.

[ELSV2] T. Ekedahl, S. Lando, M. Shapiro, and A. Vainshtein, Hurwitz numbers and intersections on moduli spaces of curves, Invent. Math. 146 (2001), 297-327.

[F1] C. Faber, A conjectural description of the tautological ring of the moduli space of curves, in Moduli of Curves and Abelian Varieties, 109-129, Aspects Math., E33, Vieweg, Braunschweig, 1999.

[F2] C. Faber, Algorithms for computing intersection numbers on moduli spaces of curves, with an application to the class of the locus of Jacobians, in New trends in algebraic geometry (Warwick, 1996), 93-109, London Math. Soc. Lecture Note Ser., 264, Cambridge Univ. Press, Cambridge, 1999.

[F3] C. Faber, MAPlE program for computing Hodge integrals, personal communication. Available at http://math.stanford.edu/ vakil/programs/.

[F4] C. Faber, personal communication, January 8, 2006.

[FP] C. Faber and R. Pandharipande, Relative maps and tautological classes, J. Eur. Math. Soc. 7 (2005), no. 1, 13-49.

[FnP] B. Fantechi and R. Pandharipande, Stable maps and branch divisors, Compositio Math. 130 (2002), no. 3, 345-364.

[Ga] A. Gathmann, Absolute and relative Gromov-Witten invariants of very ample hypersurfaces, Duke Math. J. 115 (2002), 171-203.

[GeP] E. Getzler and R. Pandharipande, Virasoro constraints and the Chern classes of the Hodge bundle, Nuclear Phys. B 530 (1998), 701-714.

[Gi1] A. Givental, Semisimple Frobenius structures at higher genus, IMRN 2001, no. 23, 1265-1286.

[Gi2] A. Givental, Gromov-Witten invariants and quantization of quadratic hamiltonians, Mosc. Math. J. 1 (2001), no. 4, 551-568, 645.

[GJ1] I. P. Goulden and D. M. Jackson, Combinatorial Enumeration, Wiley, New York, 1983 (Reprinted by Dover, 2004).

[GJ2] I. P. Goulden and D. M. Jackson, Transitive factorizations into transpositions and holomorphic mappings on the sphere, Proc. Amer. Math. Soc. 125 (1997), 51-60.

[GJVn] I. P. Goulden, D. M. Jackson and A. Vainshtein, The number of ramified coverings of the sphere by the torus and surfaces of higher genera, Annals of Combinatorics, 4 (2000), 27-46.

[GJV1] I. P. Goulden, D. M. Jackson, R. Vakil, The Gromov-Witten potential of a point, Hurwitz numbers, and Hodge integrals, Proc. London Math. Soc. (3) 83 (2001), 563-581.

[GJV2] I. P. Goulden, D. M. Jackson, R. Vakil, Towards the geometry of double Hurwitz numbers, Adv. Math. 198 (2005), 43-92.

[GJV3] I. P. Goulden, D. M. Jackson, R. Vakil, A short proof of the $\lambda_{g}$-Conjecture without Gromov-Witten theory: Hurwitz theory and the moduli of curves, math.AG/0604297, submitted.

[GrP] T. Graber and R. Pandharipande, Localization of virtual classes, Invent. Math. 135 (1999), 487-518.

[GV1] T. Graber and R. Vakil, On the tautological ring of $\overline{\mathcal{M}}_{g, n}$, in Proceedings of the Seventh Gökova GeometryTopology Conference 2000, International Press, 2000.

[GV2] T. Graber and R. Vakil, Hodge integrals, Hurwitz numbers, and virtual localization, Composition Math. 135 (1) (January 2003), 25-36.

[GV3] T. Graber and R. Vakil, Relative virtual localization and vanishing of tautological classes on moduli spaces of curves, Duke Math. J. 130 (2005), no. 1, 1-37.

[H] K. Hori, S. Katz, A. Klemm, R. Pandharipande, R. Thomas, C. Vafa, R. Vakil, and E. Zaslow, GromovWitten Theory and Mirror Symmetry, Clay Math. Inst., Amer. Math. Soc., 2002.

[IP1] E.-N. Ionel and T. H. Parker, Relative Gromov-Witten invariants, Ann. of Math. (2) 157 (2003), 45-96.

[IP2] E.-N. Ionel and T. H. Parker, The symplectic sum formula for Gromov-Witten invariants, Ann. of Math. (2), 159 (2004), 935-1025.

[KL] M. E. Kazarian and S. K. Lando, An algebro-geometric proof of Witten's conjecture, Max-Planck Institute preprint MPIM 2005-55 (2005), http://www.mpim-bonn.mpg.de/preprints, math.AG/0601760.

[LP] Y.-P. Lee and R. Pandharipande, Frobenius manifolds, Gromov-Witten theory, and Virasoro constraints, book in preparation.

[LR] A.-M. Li and Y. Ruan, Symplectic surgery and Gromov-Witten invariants of Calabi-Yau 3-folds, Invent. Math. 145 (2001), 151-218.

[Li1] J. Li, Stable morphisms to singular schemes and relative stable morphisms, J. Diff. Geom. 57 (2001), 509-578.

[Li2] J. Li, A degeneration formula of $G W$-invariants, J. Diff. Geom. 60 (2002), 199-293. 
[LX] K. Liu and H. Xu, New properties of the intersection numbers on moduli spaces of curves, preprint 2006, math.AG/0609367v2.

[LLZ] C.-C. M. Liu, K. Liu, and J. Zhou, A proof of a conjecture of Mariño-Vafa on Hodge integrals, J. Diff. Geom. 65 (2004), 289-340.

[Lo] E. Looijenga, On the tautological ring of $\mathcal{M}_{g}$, Invent. Math. 121 (1995), no. 2, 411-419.

[OP] A. Okounkov and R. Pandharipande, Gromov-Witten theory, Hurwitz numbers, and matrix models, I, math.AG/0101147.

[P1] R. Pandharipande, Hodge integrals and degenerate contributions, Comm. Math. Phys. 208 (1999), no. 2, 489-506.

[P2] R. Pandharipande, Three questions in Gromov-Witten theory, in Proceedings of the International Congress of Mathematicians, Vol. II (Beijing, 2002), 503-512, Higher Ed. Press, Beijing, 2002.

[SSV] S. Shadrin, M. Shapiro, and A. Vainshtein, On double Hurwitz numbers in genus 0, preprint 2006.

[SZ] S. Shadrin and D. Zvonkine, Changes of variables in ELSV-type formulas, preprint, 2006.

[V1] R. Vakil, The moduli space of curves and its tautological ring, Notices of the Amer. Math. Soc. (feature article), vol. 50, no. 6, June/July 2003, p. 647-658.

[V2] R. Vakil, The moduli space of curves and Gromov-Witten theory, math.AG/0602347 2 , submitted.

Department of Combinatorics and Optimization, University of Waterloo

Department of Combinatorics and Optimization, University of Waterloo

Department of Mathematics, Stanford University 